Supporting Information for:

\title{
Zirconium-Catalyzed Hydroaminoalkylation of Alkynes for the Synthesis of Allylic Amines
}

\author{
Erick Nuñez Bahenat, Samuel E. Griffin ${ }^{*}$ and Laurel L. Schafer*
}

Department of Chemistry, University of British Columbia, 2036 Main Mall, Vancouver, British

Columbia, Canada V6T 1Z1

\section{Table of Contents}

$\begin{array}{ll}\text { General Considerations } & \text { S2 }\end{array}$

$\begin{array}{ll}\text { Synthesis of Complexes } & \text { S4 }\end{array}$

Initial Catalytic Results and Evaluation of Reported Hydroaminoalkylation Catalysts $\quad$ S7

Catalytic Hydroaminoalkylation of Alkynes $\quad$ S9

Proposed General Catalytic Cycle for Hydroaminoalkylation of Alkynes $\quad$ S14

NMR Spectra $\quad$ S15

$\begin{array}{ll}\text { Crystallographic details } & \text { S37 }\end{array}$

$\begin{array}{ll}\text { References } & \text { S44 }\end{array}$ 


\section{General Considerations}

All air and moisture sensitive compounds were manipulated under inert $\mathrm{N}_{2}$ atmosphere using an MBraun LABmaster glovebox or standard Schlenk techniques. Glassware and Teflon ${ }^{\circledR}$ coated magnetic stir bars were dried in a $160{ }^{\circ} \mathrm{C}$ oven for at least 4 hours prior to transferring to the glovebox or Schlenk manifold. Toluene and hexanes were passed through an activated alumina column under $\mathrm{N}_{2}$ gas, collected in a Teflon sealed Straus flask, and sparged with $\mathrm{N}_{2}$ for 30 minutes prior to use. Benzene- $d_{6}$, and toluene- $d_{8}$ were dried over sodium metal and distilled under $\mathrm{N}_{2}$ and collected in a Teflon sealed Straus flask prior to use. Diatomaceous earth was dried in an oven at $160^{\circ} \mathrm{C}$ for at least 24 hours before transferring to the glovebox. J. Young NMR tubes ( 8 " x $5 \mathrm{~mm}$ ) with Teflon screw-caps were used for NMR reactions. ${ }^{1} \mathrm{H}$ NMR and ${ }^{13} \mathrm{C}\left\{{ }^{1} \mathrm{H}\right\}$ spectra were collected using a Bruker $300 \mathrm{MHz}$ or $400 \mathrm{MHz}$ Avance spectrometer at $298 \mathrm{~K}$ unless otherwise noted. Chemical shifts, $\delta$, are reported relative to the corresponding residual protio solvent in parts per million (ppm). Coupling constants, $J$, are given in $\operatorname{Hertz}(\mathrm{Hz})$. Signal multiplicity is reported using the following abbreviations: $\mathrm{s}=$ singlet, $\mathrm{d}=$ doublet, $\mathrm{t}=$ triplet, $\mathrm{q}=$ quartet, $\mathrm{sep}=$ septet, $\mathrm{m}=$ multiplet, and $\mathrm{br}=$ broad. For NMR yield determinations, $\mathrm{T}_{1}$ relaxation times for the relevant signals were estimated using a spin-echo pulse sequence and relaxation delays were extended accordingly during ${ }^{1} \mathrm{H}$ NMR data collection. The internal standard used for quantitative ${ }^{1} \mathrm{H}$ NMR experiments was 1,3,5-trimethoxybenzene and the chemical shifts associated with it are the following: ${ }^{1} \mathrm{H}$ NMR $\left(\mathrm{C}_{6} \mathrm{D}_{6}, 400 \mathrm{MHz}\right) \delta=6.25(\mathrm{~s}, 3 \mathrm{H})$, $3.32(\mathrm{~s}, 9 \mathrm{H})$. Mass spectra (MS) were collected using either a Jeol AccuTOF GCv 4G spectrometer equipped with a Liquid Injection Field Desorption Ionization (LIFDI) probe or an Agilent 6545 QTOF spectrometer equipped with an electrospray or field desorption ionization source, with fragments given in mass per charge number $(\mathrm{m} / \mathrm{z})$. Elemental analysis $($ EA) was performed with a Thermo Flash 2000 Elemental Analyzer. The elemental composition values are given in percentages (\%). Single crystal X-ray diffraction data were collected using a Bruker X8 APEX or Bruker APEX DUO diffractometer. All chemicals were purchased from commercial sources and used without further purification, and those which were not already dried and stored under inert atmosphere were either dried over calcium hydride and degassed via the freeze-pump-thaw method or sublimed or dried under high vacuum before use. All liquid amines were dried over molecular sieves before degassing via the freeze-pump-thaw method and bringing them into the glovebox. All solid amines were dried and degassed in a drying tube before bringing them into the glovebox. All solid alkynes were sublimed or dried under high vacuum before use. All liquid alkynes were dried over calcium hydride and distilled under vacuum before degassing via the freeze-pumpthaw method and bringing them into the glovebox. The bis(urea) proligand $\mathbf{L 1},{ }^{1}$ dibenzyl complex $\mathbf{D},{ }^{2}$ sodium ureate salt $\mathbf{L 2},{ }^{3} \mathrm{Ta}\left(\mathrm{CH}_{2} \mathrm{SiMe}_{3}\right)_{2} \mathrm{Cl}_{2},{ }^{4}$ and titanium formamidinate complex $\mathbf{E}^{5}$ were prepared 
according to literature procedures. All alkynes ${ }^{6,7}$ and amines ${ }^{8,9}$ that were not commercially available were synthesized according to literature methods. 


\section{Synthesis of Complexes}

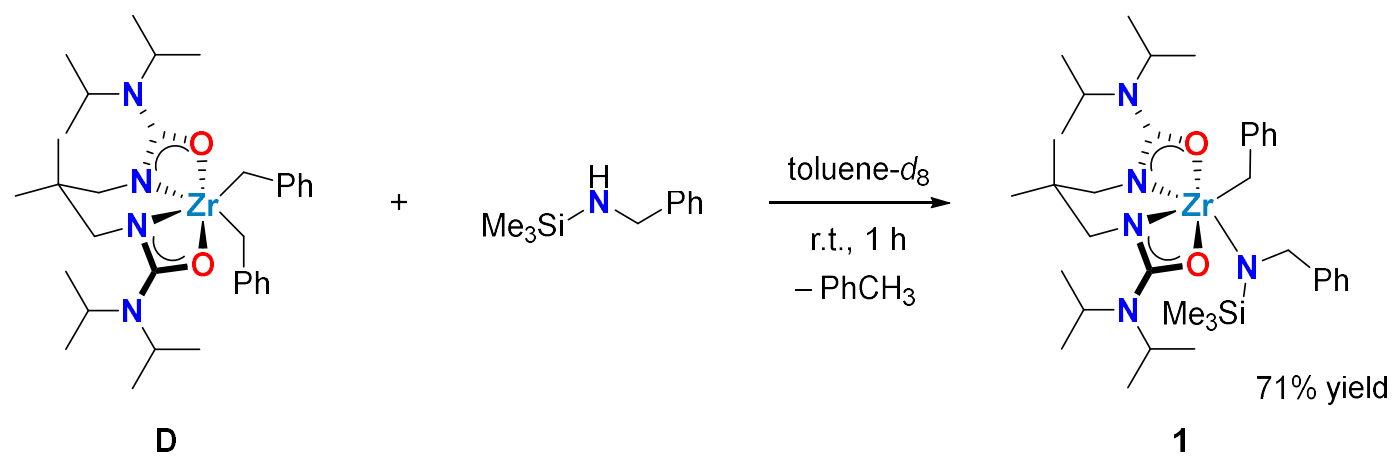

Synthesis of 1: A $20 \mathrm{~mL}$ vial was charged with dibenzyl complex D (0.104 g, $0.166 \mathrm{mmol})$ before dissolving in $\sim 5 \mathrm{~mL}$ toluene with vigorous swirling to give a yellow solution. Then, a solution of $N$ (trimethylsilyl)benzylamine $(0.030 \mathrm{~g}, 0.17 \mathrm{mmol})$ in $\sim 3 \mathrm{~mL}$ toluene was added dropwise while stirring. The solution turned gradually to pale yellow over a period of $1 \mathrm{~h}$. After this time, the volatiles were removed under vacuum and a white powder contaminated with a yellow impurity was obtained. This reaction mixture was re-dissolved in $\sim 5 \mathrm{~mL}$ hexanes with gentle heating, resulting in a yellow solution. Cooling this solution to $-35{ }^{\circ} \mathrm{C}$ for $24 \mathrm{~h}$ afforded $0.084 \mathrm{~g}$ of $\mathbf{1}$ as colorless crystals ( $71 \%$ yield). These crystals were suitable for X-ray diffraction. ${ }^{1} \mathrm{H}$ NMR $\left(\mathrm{C}_{6} \mathrm{D}_{6}, 400 \mathrm{MHz}\right) \delta=7.64(\mathrm{~m}, 2 \mathrm{H}), 7.48(\mathrm{~m}, 2 \mathrm{H}), 7.27(\mathrm{~m}, 2 \mathrm{H}), 7.20(\mathrm{~m}, 2 \mathrm{H})$, $7.13(\mathrm{~m}, 1 \mathrm{H}), 6.83(\mathrm{~m}, 1 \mathrm{H}), 4.78(\mathrm{~s}, 2 \mathrm{H}), 3.46(\mathrm{sep}, J=6.7 \mathrm{~Hz}, 4 \mathrm{H}), 3.03(\mathrm{~d}, J=11.4 \mathrm{~Hz}, 2 \mathrm{H}), 2.87(\mathrm{~d}, J=$ $11.4 \mathrm{~Hz}, 2 \mathrm{H}), 2.83(\mathrm{~s}, 2 \mathrm{H}), 1.31$ (d, $J=6.6 \mathrm{~Hz}, 12 \mathrm{H}), 1.21(\mathrm{~d}, J=6.7 \mathrm{~Hz}, 12 \mathrm{H}), 0.72(\mathrm{~s}, 3 \mathrm{H}), 0.54(\mathrm{~s}, 3 \mathrm{H})$, $0.11(\mathrm{~s}, 9 \mathrm{H}) ;{ }^{13} \mathrm{C} \mathrm{NMR}\left(\mathrm{C}_{6} \mathrm{D}_{6}, 100 \mathrm{MHz}\right) \delta=170.3,149.8,145.2,128.7,126.9,128.4-127.8(2 \mathrm{C}), 126.3$, 120.1, 61.1, 57.4, 47.1, 45.0, 36.0, 27.0, 22.7, 22.6, 21.8, 1.4. The molecular ion was not observed by MS, instead the ion corresponding to a loss of a benzyl group was observed: MS(LIFDI) $m / z 622\left[\mathrm{M}-\mathrm{CH}_{2} \mathrm{Ph}\right]^{+}$, $m / z 535\left[\mathrm{M}-\mathrm{N}\left(\mathrm{SiMe}_{3}\right)\left(\mathrm{CH}_{2} \mathrm{Ph}\right)\right]^{+}$. Satisfactory elemental analysis could not be obtained: Anal Calcd for $\mathrm{C}_{36} \mathrm{H}_{61} \mathrm{~N}_{5} \mathrm{O}_{2} \mathrm{SiZr}: \mathrm{N}, 9.79 ; \mathrm{C}, 60.46 ; \mathrm{H}, 8.60$. Found: N, 9.81; C, 59.36; H, 8.39.

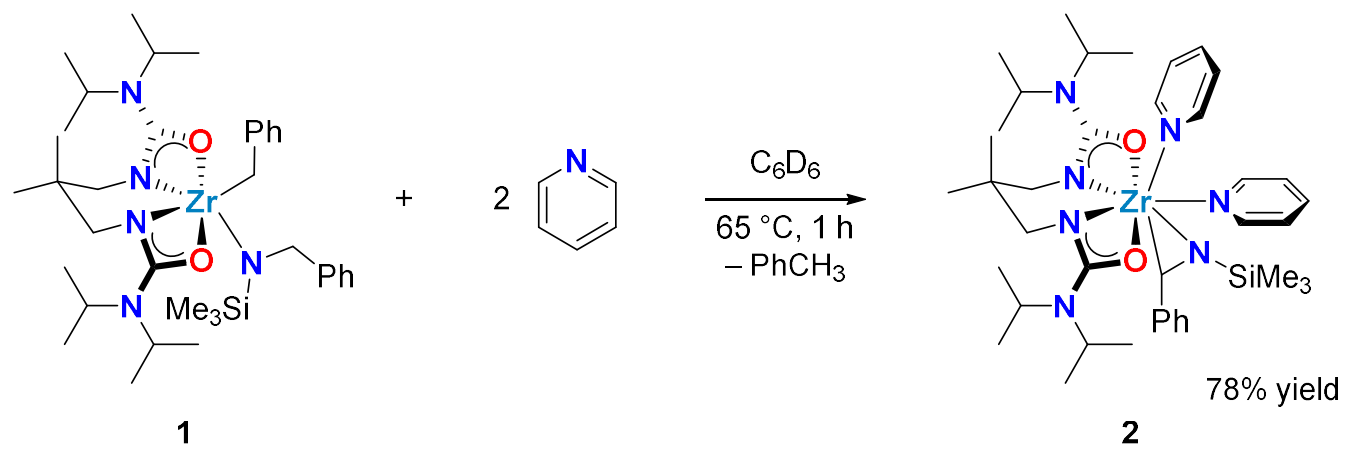


Synthesis of 2: To a $\sim 2 \mathrm{~mL} \mathrm{C}_{6} \mathrm{D}_{6}$ solution of complex 1 ( $\left.0.093 \mathrm{~g}, 0.13 \mathrm{mmol}\right)$, pyridine $(0.022 \mathrm{~g}, 0.28$ mmol) was added using $\sim 0.5 \mathrm{~mL} \mathrm{C}_{6} \mathrm{D}_{6}$ for quantitative transfer, causing a change from colorless to bright yellow. ${ }^{1} \mathrm{H}$ NMR spectroscopy revealed the formation of a pyridine adduct of 1 . Heating the reaction to 65 ${ }^{\circ} \mathrm{C}$ gradually resulted in a change of color to dark red over a period of $30 \mathrm{~min}$. The reaction was found to be complete after $1 \mathrm{~h}$ by ${ }^{1} \mathrm{H}$ NMR spectroscopy. Next, the solution was transferred to a $20 \mathrm{~mL}$ vial and the volatiles were removed in vacuo. The resulting dark red powder was dissolved in $\sim 5 \mathrm{~mL}$ hexanes and the solution was cooled down to $-35{ }^{\circ} \mathrm{C}$ overnight, during which time dark red crystals suitable for X-ray diffraction precipitated. The supernatant was then removed and $0.079 \mathrm{~g}$ of 2 (78\% yield) were isolated. ${ }^{1} \mathrm{H}$ $\operatorname{NMR}\left(\mathrm{C}_{6} \mathrm{D}_{6}, 300 \mathrm{MHz}\right) \delta=9.26$ (br m, 2H), 8.74 (br m, 2H), 7.29 (m, 4H), 6.85 (m, 3H), 6.65 (br m, 4H), 4.18 (s, 1H), 3.55 (br m, 5H), 3.20 (br d, $J=11.7 \mathrm{~Hz}, 1 \mathrm{H}), 2.97$ (br d, $J=11.7 \mathrm{~Hz}, 1 \mathrm{H}), 2.83$ (br d, $J=11.7$ $\mathrm{Hz}, 1 \mathrm{H}), 1.36$ (br m, 12H), 1.17 (br m, 12H), 0.80 (br s, 3H), 0.77 (br s, 3H), 0.24 (s, 9H). ${ }^{13} \mathrm{C} \mathrm{NMR}\left(\mathrm{C}_{6} \mathrm{D}_{6}\right.$, $100 \mathrm{MHz}) \delta=165.8,159.7,150.12,128.6-127.6$ (1C), 127.2, 123.5, 121.1, 116.4, 73.8, 56.7, 56.3, 46.8, 46.7, 36.8, 35.0, 34.9, 25.7, 22.9, 22.4, 20.9, 2.5. MS(LIFDI) $m / z 779[\mathrm{M}]^{+}, m / z 621\left[\mathrm{M}-\left(\mathrm{NC}_{5} \mathrm{H}_{5}\right)_{2}\right]^{+}$. Satisfactory elemental analysis could not be obtained, possibly due to loss of pyridine when removing volatiles: Anal Calcd for $\mathrm{C}_{39} \mathrm{H}_{63} \mathrm{~N}_{7} \mathrm{O}_{2} \mathrm{SiZr}$ : N, 12.55; C, 59.96; H, 8.13. Found: $\mathrm{N}, 11.86 ; \mathrm{C}, 60.36 ; \mathrm{H}, 8.47$.

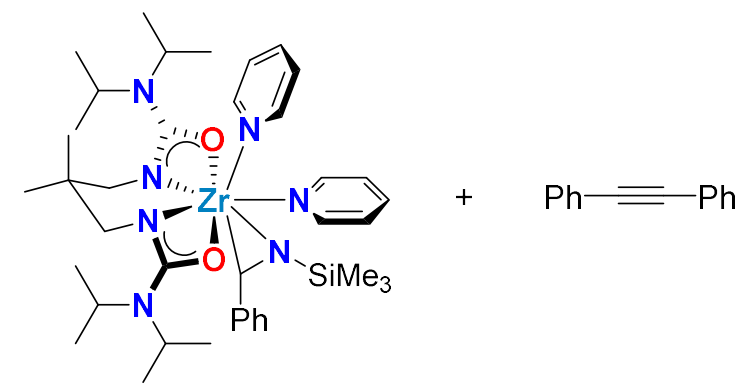

2

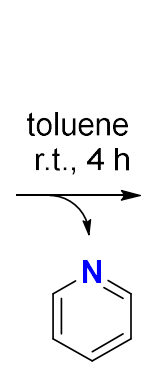

Synthesis of 3: A $20 \mathrm{~mL}$ vial was charged with complex $2(0.084 \mathrm{~g}, 0.11 \mathrm{mmol})$ before dissolving in $\sim 5$ $\mathrm{mL}$ toluene with vigorous swirling to give a dark red solution. Then, a solution of diphenylacetylene $(0.019$ $\mathrm{g}, 0.11 \mathrm{mmol})$ in $\sim 5 \mathrm{~mL}$ toluene was added to the solution of 2 while stirring. The solution turned gradually to orange over a period of $1.5 \mathrm{~h}$ and then turned pale yellow after a total of $3.5 \mathrm{~h}$. The volatiles were then removed in vacuo and a white powder contaminated with an orange impurity was obtained. This reaction mixture was re-dissolved in $\sim 8 \mathrm{~mL}$ hexanes at room temperature to give a clear, yellow solution. Cooling this solution to $-35{ }^{\circ} \mathrm{C}$ for $24 \mathrm{~h}$ afforded $0.065 \mathrm{~g}$ of $\mathbf{3}(68 \%$ yield) as colorless crystals suitable for X-ray diffraction. ${ }^{1} \mathrm{H}$ NMR $\left(\mathrm{C}_{6} \mathrm{D}_{6}, 300 \mathrm{MHz}\right) \delta=8.70(\mathrm{~d}, J=7.5 \mathrm{~Hz}, 2 \mathrm{H}), 7.92(\mathrm{~d}, J=7.5 \mathrm{~Hz}, 2 \mathrm{H}), 7.25(\mathrm{t}, J=$ $7.4 \mathrm{~Hz}, 2 \mathrm{H}), 7.11(\mathrm{~m}, 1 \mathrm{H}), 7.03(\mathrm{~m}, 2 \mathrm{H}), 6.95(\mathrm{~m}, 2 \mathrm{H}), 6.89(\mathrm{~m}, 2 \mathrm{H}), 6.76(\mathrm{~m}, 4 \mathrm{H}), 6.48(\mathrm{~m}, 3 \mathrm{H}), 5.72(\mathrm{~s}$, 1H), 3.56 (br m, 4H), 3.00 (br m, 4H), 1.35 (br m, 24H), 0.67 (br s, 3H), 0.45 (br s, 3H), 0.26 (s, 9H). ${ }^{13} \mathrm{C}$ NMR $\left(\mathrm{C}_{6} \mathrm{D}_{6}, 100 \mathrm{MHz}\right) \delta=191.1,150.9,150.3,144.8,137.0,130.3,130.2,129.3,128.6,127.4,126.9$, 
$126.4,126.1,125.7,124.6,123.1,120.0,74.5,56.9,47.1,37.1,32.0,22.2,14.4,3.0$. The molecular ion was not observed by MS, instead the ion corresponding to a loss of pyridine was observed: MS(LIFDI) $\mathrm{m} / z 799$ $\left[\mathrm{M}-\left(\mathrm{NC}_{5} \mathrm{H}_{5}\right)\right]^{+}$. Satisfactory elemental analysis could not be obtained, possibly due to loss pyridine during removal of volatiles: Anal Calcd for $\mathrm{C}_{48} \mathrm{H}_{68} \mathrm{~N}_{6} \mathrm{O}_{2} \mathrm{SiZr}$ : N, 9.55; C, 65.48; H, 7.79. Found: N, 9.03; C, 63.65; $\mathrm{H}, 8.30$.

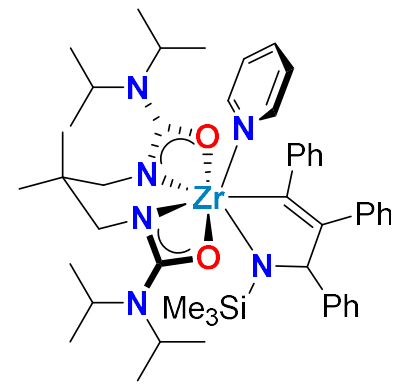

3

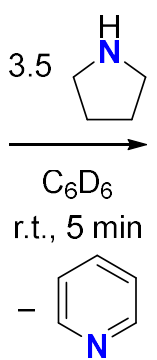

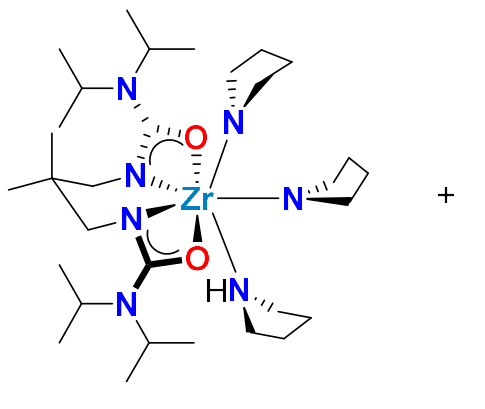

4

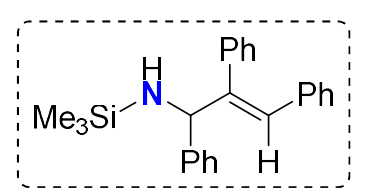

$5 a$

Synthesis of 5a: To a solution of complex $3(0.010 \mathrm{~g}, 0.011 \mathrm{mmol})$ in $\sim 1 \mathrm{~mL} \mathrm{C}_{6} \mathrm{D}_{6}$, pyrrolidine was added ( $0.003 \mathrm{~g}, 0.04 \mathrm{mmol})$ with $\sim 0.5 \mathrm{~mL} \mathrm{C}_{6} \mathrm{D}_{6}$ to ensure quantitative transfer, turning the solution from pale yellow to colorless. After $5 \mathrm{~min},{ }^{1} \mathrm{H}$ NMR spectroscopy revealed the formation of previously reported complex $4,{ }^{10}$ free pyridine, and allylic amine 5a. Characterization of 5a was performed in situ. ${ }^{1} \mathrm{H}$ NMR $\left(\mathrm{C}_{6} \mathrm{D}_{6}, 300 \mathrm{MHz}\right) \delta=7.37(\mathrm{~m}, 2 \mathrm{H}), 7.19(\mathrm{~m}, 2 \mathrm{H}), 7.07(\mathrm{~m}, 3 \mathrm{H}), 6.96(\mathrm{~m}, 8 \mathrm{H}), 6.84(\mathrm{~s}, 1 \mathrm{H}), 4.90(\mathrm{~d}, J=$ $10.8 \mathrm{~Hz}, 1 \mathrm{H}), 0.08(\mathrm{~s}, 9 \mathrm{H}) ;{ }^{13} \mathrm{C} \mathrm{NMR}\left(\mathrm{C}_{6} \mathrm{D}_{6}, 100 \mathrm{MHz}\right) \delta=147.3,145.3,140.3,137.6,129.8,129.6,128.6$, $128.5,128.4-127.8(2 \mathrm{C}), 127.4,127.3,127.0,126.9,64.2,0.5$. 


\section{Initial Catalytic Results and Evaluation of Reported Hydroaminoalkylation Catalysts}
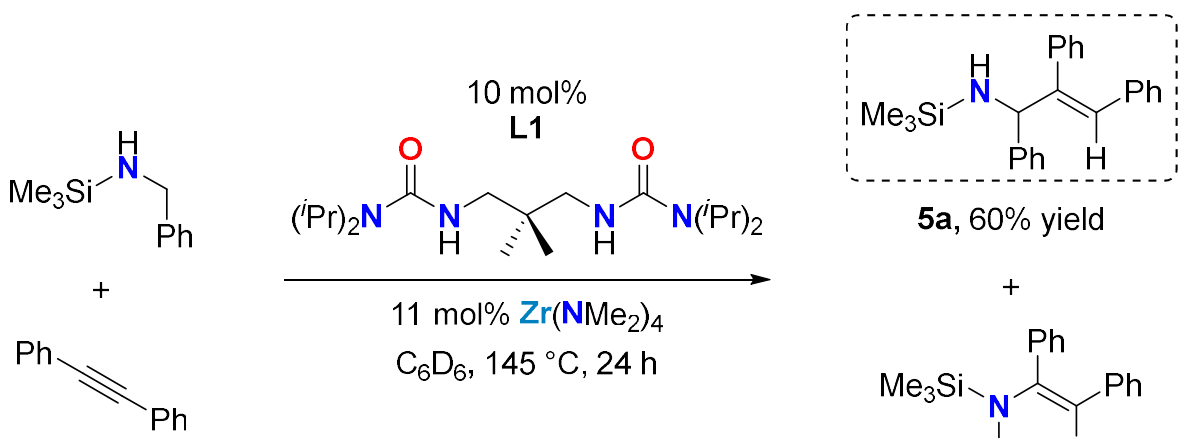

$5 a, 60 \%$ yield

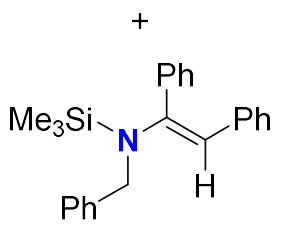

$5 b, 14 \%$ yield

Hydroaminoalkylation with $\boldsymbol{N}$-(trimethylsilyl)benzylamine: To a small vial containing ligand L1 (0.004

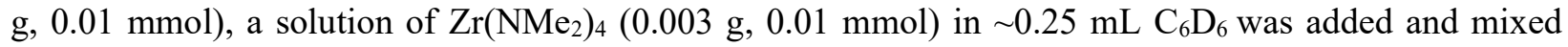
thoroughly to form the precatalyst. After $20 \mathrm{~min}$, solutions of $N$-(trimethylsilyl)benzylamine $(0.018 \mathrm{~g}, 0.10$ mmol) and diphenylacetylene $(0.018 \mathrm{~g}, 0.10 \mathrm{mmol})$ in $\sim 0.25 \mathrm{~mL} \mathrm{C}_{6} \mathrm{D}_{6}$ were added separately to the precatalyst solution, using a total of $\sim 0.25 \mathrm{~mL} \mathrm{C}_{6} \mathrm{D}_{6}$ for quantitative transfer. The solution was then transferred into a $\mathrm{J}$. Young tube and a $\mathrm{t}=0{ }^{1} \mathrm{H}$ NMR spectrum was obtained before heating to $145{ }^{\circ} \mathrm{C}$ for $24 \mathrm{~h}$. After the reaction, a ${ }^{1} \mathrm{H}$ NMR spectrum was obtained (vide infra). A solution of 1,3,5trimethoxybenzene in $\sim 0.15 \mathrm{~mL} \mathrm{C}_{6} \mathrm{D}_{6}$ was then transferred to the reaction mixture and a ${ }^{1} \mathrm{H}$ NMR spectrum was obtained for NMR yield determination.

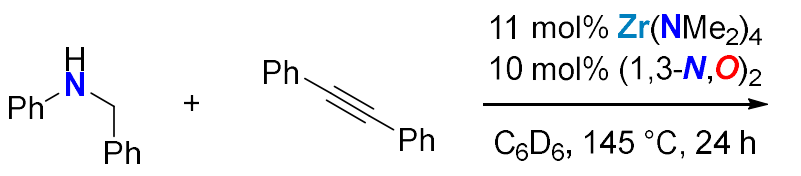

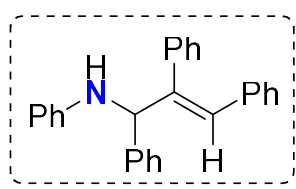

6a, $96 \%$ conversion<smiles>C(=C(\c1ccccc1)N(c1ccccc1)c1ccccc1)\c1ccccc1</smiles>

6b, not observed

Hydroaminoalkylation with $\boldsymbol{N}$-benzylaniline: To a small vial containing ligand L1 $(0.001 \mathrm{~g}, 0.004$ $\mathrm{mmol})$, a solution of $\mathrm{Zr}\left(\mathrm{NMe}_{2}\right)_{4}(0.001 \mathrm{~g}, 0.004 \mathrm{mmol})$ in $\sim 0.25 \mathrm{~mL} \mathrm{C}_{6} \mathrm{D}_{6}$ was added and mixed thoroughly to form the precatalyst. After $20 \mathrm{~min}$, solutions of $N$-benzylaniline $(0.007 \mathrm{~g}, 0.04 \mathrm{mmol})$ and diphenylacetylene $(0.007 \mathrm{~g}, 0.04 \mathrm{mmol})$ in $\sim 0.25 \mathrm{~mL} \mathrm{C}_{6} \mathrm{D}_{6}$ were added separately to the precatalyst solution, using a total of $\sim 0.25 \mathrm{~mL} \mathrm{C}_{6} \mathrm{D}_{6}$ for quantitative transfer. The solution was then transferred into a J. Young tube and a $\mathrm{t}=0{ }^{1} \mathrm{H}$ NMR spectrum was obtained before heating to $145^{\circ} \mathrm{C}$ for $24 \mathrm{~h}$. After the reaction, a ${ }^{1} \mathrm{H}$ NMR spectrum was obtained to determine the conversion to the allylic amine product. Note: changing the solvent (for example to toluene) and/or lowering the reaction temperature (for example to $140{ }^{\circ} \mathrm{C}$ ) dramatically reduced the conversion by approximately $20 \%$ in either case. 


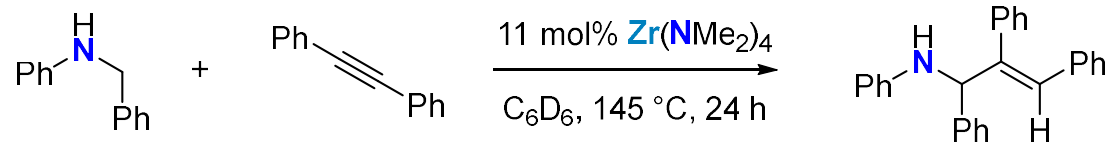

6a, $15 \%$ conversion

Hydroaminoalkylation with $N$-benzylaniline (No Ligand): To a small vial containing a solution of $\mathrm{Zr}\left(\mathrm{NMe}_{2}\right)_{4}(0.001 \mathrm{~g}, 0.004 \mathrm{mmol})$ in $\sim 0.25 \mathrm{~mL} \mathrm{C}_{6} \mathrm{D}_{6}$, solutions of $N$-benzylaniline $(0.007 \mathrm{~g}, 0.04 \mathrm{mmol})$ and diphenylacetylene $(0.007 \mathrm{~g}, 0.04 \mathrm{mmol})$ in $\sim 0.25 \mathrm{~mL} \mathrm{C}_{6} \mathrm{D}_{6}$ were added separately using a total of $\sim 0.25$ $\mathrm{mL} \mathrm{C}_{6} \mathrm{D}_{6}$ for quantitative transfer. The resulting solution was then transferred into a $\mathrm{J}$. Young tube and a $\mathrm{t}$ $=0{ }^{1} \mathrm{H}$ NMR spectrum was obtained before heating to $145{ }^{\circ} \mathrm{C}$ for $24 \mathrm{~h}$. After the reaction, a ${ }^{1} \mathrm{H}$ NMR spectrum was obtained to determine the conversion to the allylic amine product.

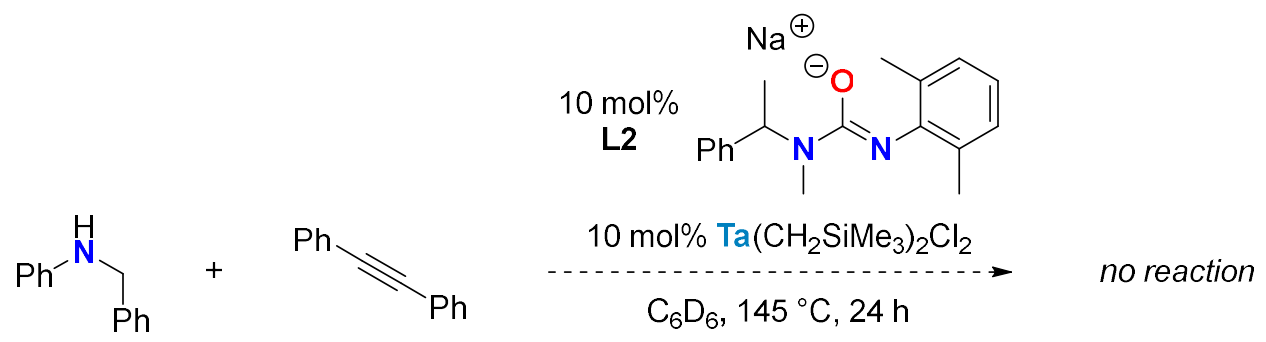

Hydroaminoalkylation with $N$-benzylaniline (Ta Catalyst): To a small vial containing ureate ligand L2 $(0.001 \mathrm{~g}, 0.004 \mathrm{mmol})$, a solution of $\mathrm{Ta}\left(\mathrm{CH}_{2} \mathrm{SiMe}_{3}\right)_{2} \mathrm{Cl}_{2}(0.002 \mathrm{~g}, 0.004 \mathrm{mmol})$ in $\sim 0.25 \mathrm{~mL} \mathrm{C}_{6} \mathrm{D}_{6}$ was added and mixed thoroughly to form the precatalyst. After $20 \mathrm{~min}$, solutions of $N$-benzylaniline $(0.007 \mathrm{~g}, 0.04$ mmol $)$ and diphenylacetylene $(0.007 \mathrm{~g}, 0.04 \mathrm{mmol})$ in $\sim 0.25 \mathrm{~mL} \mathrm{C}_{6} \mathrm{D}_{6}$ were added separately to the precatalyst solution, using a total of $\sim 0.25 \mathrm{~mL} \mathrm{C}_{6} \mathrm{D}_{6}$ for quantitative transfer. The solution was then transferred into a $\mathrm{J}$. Young tube and a $\mathrm{t}=0{ }^{1} \mathrm{H}$ NMR spectrum was obtained before heating to $145{ }^{\circ} \mathrm{C}$ for $24 \mathrm{~h}$. After the reaction, a ${ }^{1} \mathrm{H}$ NMR spectrum was obtained and revealed that no reaction occurred between the two substrates.

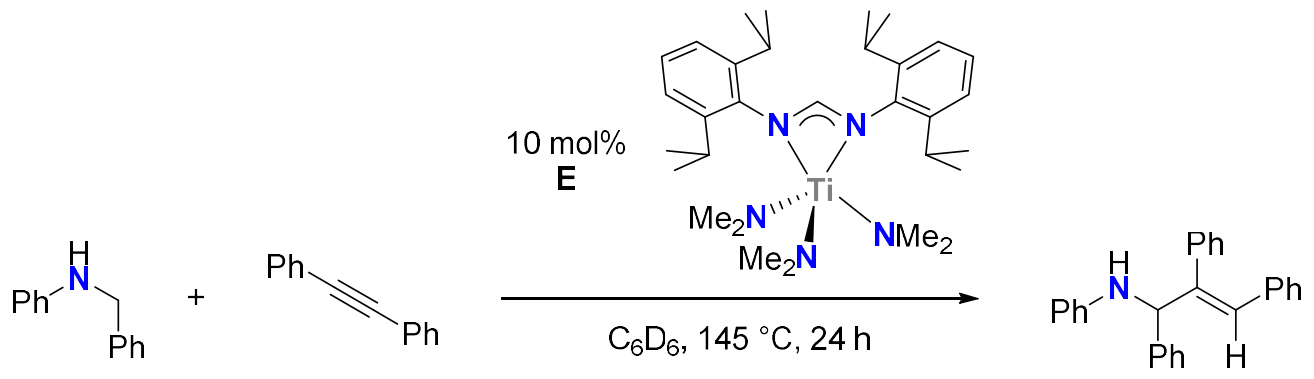

$6 a, 25 \%$ conversion

Hydroaminoalkylation with $N$-benzylaniline (Ti Catalyst): To a small vial containing a solution of $\mathrm{Ti}$ catalyst $\mathbf{E}(0.002 \mathrm{~g}, 0.004 \mathrm{mmol})$ in $\sim 0.25 \mathrm{~mL} \mathrm{C}_{6} \mathrm{D}_{6}$, solutions of $N$-benzylaniline $(0.007 \mathrm{~g}, 0.04 \mathrm{mmol})$ and 
diphenylacetylene $(0.007 \mathrm{~g}, 0.04 \mathrm{mmol})$ in $\sim 0.25 \mathrm{~mL} \mathrm{C}_{6} \mathrm{D}_{6}$ were added separately using a total of $\sim 0.25 \mathrm{~mL}$ $\mathrm{C}_{6} \mathrm{D}_{6}$ for quantitative transfer. The resulting solution was then transferred into a $\mathrm{J}$. Young tube and a $\mathrm{t}=0$ ${ }^{1} \mathrm{H}$ NMR spectrum was obtained before heating to $145^{\circ} \mathrm{C}$ for $24 \mathrm{~h}$. After the reaction, a ${ }^{1} \mathrm{H}$ NMR spectrum was obtained to determine the conversion to the allylic amine product.

\section{Catalytic Hydroaminoalkylation of Alkynes}

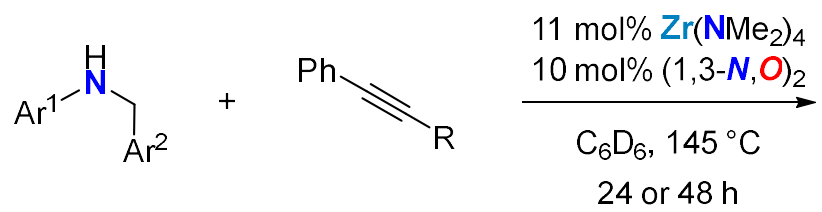

General Procedure for Alkyne Hydroaminoalkylation: To a small vial containing ligand L1 (0.004 g, $0.01 \mathrm{mmol})$, a solution of $\mathrm{Zr}\left(\mathrm{NMe}_{2}\right)_{4}(0.003 \mathrm{~g}, 0.01 \mathrm{mmol})$ in $\sim 0.25 \mathrm{~mL} \mathrm{C} \mathrm{C}_{6}$ was added and mixed thoroughly to form the precatalyst. After $20 \mathrm{~min}$, solutions of the respective amine $(0.10 \mathrm{mmol})$ and alkyne $(0.10 \mathrm{mmol})$ in $\sim 0.25 \mathrm{~mL} \mathrm{C}_{6} \mathrm{D}_{6}$ were added separately to the precatalyst solution, using a total of $\sim 0.25 \mathrm{~mL}$ $\mathrm{C}_{6} \mathrm{D}_{6}$ for quantitative transfer. The solution was then transferred into a J. Young tube and a $\mathrm{t}=0{ }^{1} \mathrm{H}$ NMR spectrum was obtained before heating to $145^{\circ} \mathrm{C}$ for 24 or $48 \mathrm{~h}$. After the reaction, a ${ }^{1} \mathrm{H}$ NMR spectrum was obtained for in situ characterization (vide infra). A solution of 1,3,5-trimethoxybenzene in $\sim 0.15 \mathrm{~mL} \mathrm{C}_{6} \mathrm{D}_{6}$ was then transferred to the reaction mixture and a ${ }^{1} \mathrm{H}$ NMR spectrum was obtained for NMR yield determination and regioisomeric ratio (a:b). Each reaction was run in duplicate. For product characterization, the crude reaction mixture without internal standard was quenched with $\sim 2 \mathrm{~mL} \mathrm{DCM}$ and filtered through diatomaceous earth. The volatiles were then removed in vacuo and the resulting residue was purified by silica column chromatography (hexanes/ethyl acetate). In some cases, the product decomposed during solvent removal after column purification. Thus, to preserve the NMR signals of the product for characterization, some solvent impurities can be observed in select NMR spectra. In cases where multiple regioisomers are produced, the isomers were isolated as a mixture.

\section{Hydroaminoalkylation of Diphenylacetylene with $N$-benzylaniline (6a)}

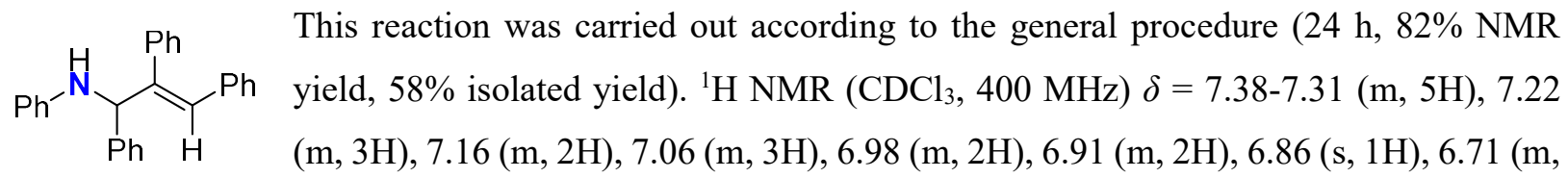

1H), $6.67(\mathrm{~m}, 2 \mathrm{H}), 5.18(\mathrm{~s}, 1 \mathrm{H}), 4.32(\mathrm{br} \mathrm{s}, 1 \mathrm{H}) ;{ }^{13} \mathrm{C} \mathrm{NMR}\left(\mathrm{CDCl}_{3}, 100 \mathrm{MHz}\right) \delta=147.2,141.8,141.1$, 
$139.4,136.7,129.5,129.4,129.3,129.2,128.8,128.7,128.3,128.0,127.8,127.5,126.9,117.9,113.8,66.2$. HRMS(ESI) $m / z$ Calcd for $\mathrm{C}_{27} \mathrm{H}_{22} \mathrm{~N}[\mathrm{M}]^{+}: 360.1752$; found: 360.1758 .

\section{Hydroaminoalkylation of 1-Methyl-4-(phenylethynyl)benzene with $N$-benzylaniline (7)}

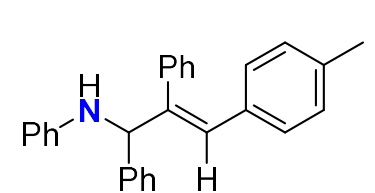

+ regioisomer

This reaction was carried out according to the general procedure $(48 \mathrm{~h}, 75 \%$ NMR yield, 1.1:1, 51\% isolated yield). ${ }^{1} \mathrm{H}$ NMR $\left(\mathrm{CDCl}_{3}, 400 \mathrm{MHz}\right) \delta=7.37$ $(\mathrm{m}, 4 \mathrm{H}), 7.32-7.25(\mathrm{~m}, 6 \mathrm{H}), 7.21(\mathrm{~m}, 2 \mathrm{H}), 7.15(\mathrm{~m}, 4 \mathrm{H}), 7.06-7.00(\mathrm{~m}, 6 \mathrm{H}), 6.98-$ $6.92(\mathrm{~m}, 4 \mathrm{H}), 6.87-6.78(\mathrm{~m}, 8 \mathrm{H}), 6.70(\mathrm{~m}, 2 \mathrm{H}), 6.65(\mathrm{~m}, 4 \mathrm{H}), 5.16(\mathrm{br} \mathrm{s}, 2 \mathrm{H})$, 4.27 (br s, 2H), 2.29 (s, 3H), $2.20(\mathrm{~s}, 3 \mathrm{H}) ;{ }^{13} \mathrm{C} \mathrm{NMR}\left(\mathrm{CDCl}_{3}, 100 \mathrm{MHz}\right) \delta=147.2,141.7,141.2,140.8$, $139.6,137.1,136.9,136.7,136.2$, 133.8, 129.4, 129.3, 129.3, 129.2, 129.0, 128.7, 128.7, 128.1, 128.2, $128.0,128.0,128.0,127.7,127.4,126.8,117.9,113.8,66.3,21.4,21.2$. HRMS(ESI) $m / z$ Calcd for $\mathrm{C}_{28} \mathrm{H}_{24} \mathrm{~N}$ $[\mathrm{M}]^{+}:$374.1909; found: 374.1912.

\section{Hydroaminoalkylation of 1-Methoxy-4-(phenylethynyl)benzene with $N$-benzylaniline (8)}

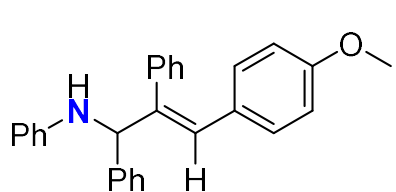

+ regioisomer

This reaction was carried out according to the general procedure (48 h, 76\% NMR yield, 1.4:1, 66\% isolated yield; some E/Z-isomerization also occurred during purification). ${ }^{1} \mathrm{H} \mathrm{NMR}\left(\mathrm{CDCl}_{3}, 400 \mathrm{MHz}\right) \delta=7.48-7.41(\mathrm{~m}, 2 \mathrm{H}), 7.40-$ $7.26(\mathrm{~m}, 4 \mathrm{H}), 7.21(\mathrm{~m}, 2 \mathrm{H}), 7.17-7.08(\mathrm{~m}, 2 \mathrm{H}), 7.06-6.86(\mathrm{~m}, 5 \mathrm{H}), 6.86-6.62$

$(\mathrm{m}, 5 \mathrm{H}), 5.22(\mathrm{~s}, 1 \mathrm{H}), 5.16(\mathrm{~s}, 1 \mathrm{H}), 4.34$ (br s, 1H), $3.84(\mathrm{~s}, 3 \mathrm{H}), 3.82(\mathrm{~s}, 3 \mathrm{H}), 3.75(\mathrm{~s}, 3 \mathrm{H}) ;{ }^{13} \mathrm{C} \mathrm{NMR}$ $\left(\mathrm{CDCl}_{3}, 100 \mathrm{MHz}\right) \delta=159.2,159.0,158.5,147.3,141.9,141.4,141.3,141.3,139.8,139.7,139.6,137.0$, $136.8,131.4,130.7,130.4,129.4,129.3,129.2$, 128.8, 128.7, 128.7, 128.2, 128.0, 128.0, 127.7, 127.7, $127.4,126.8,126.8,117.9,114.1,113.8,113.5,66.3,65.7,55.4,55.2$. HRMS(FD) $m / z$ Calcd for $\mathrm{C}_{28} \mathrm{H}_{25} \mathrm{NO}$ $[\mathrm{M}]^{+}: 391.19361$; found: 391.19472 .

Hydroaminoalkylation of 1-(Phenylethynyl)-4-(trifluoromethyl)benzene with $\mathrm{N}$-benzylaniline (9)

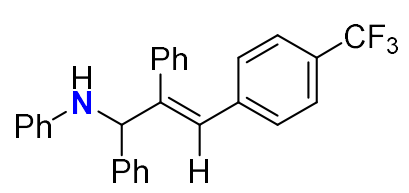

+ regioisomer

This reaction was carried out according to the general procedure $(48 \mathrm{~h}, 63 \%$ NMR yield, $1: 1,61 \%$ isolated yield). ${ }^{1} \mathrm{H}$ NMR $\left(\mathrm{CDCl}_{3}, 300 \mathrm{MHz}\right) \delta=7.48$ $(\mathrm{m}, 2 \mathrm{H}), 7.42-7.30(\mathrm{~m}, 13 \mathrm{H}), 7.30-7.18(\mathrm{~m}, 7 \mathrm{H}), 7.15-6.82(\mathrm{~m}, 12 \mathrm{H}), 6.78(\mathrm{~m}$, 2H) $6.73(\mathrm{~m}, 4 \mathrm{H}), 5.20(\mathrm{~s}, 1 \mathrm{H}), 5.19(\mathrm{~s}, 1 \mathrm{H}), 4.36(\mathrm{br} \mathrm{s}, 2 \mathrm{H}) ;{ }^{13} \mathrm{C} \mathrm{NMR}\left(\mathrm{C}_{6} \mathrm{D}_{6}\right.$,

$100 \mathrm{MHz}) \delta=147.0,147.0,144.4,143.4,140.6,140.4,138.8,136.1,129.7,129.5,129.5,129.4,129.4$, $129.4,129.3,129.0,128.9,128.9,128.8,128.7,128.4,128.1,128.0,128.0,127.9,127.3,126.6,125.6$ (q, $J$ $=3.8 \mathrm{~Hz}), 124.9(\mathrm{q}, J=3.8 \mathrm{~Hz}), 122.9,118.2,118.2,113.8,66.3,66.0 ;{ }^{19} \mathrm{~F} \mathrm{NMR}\left(\mathrm{CDCl}_{3}, 282 \mathrm{MHz}\right) \delta=$ 63.0, 62.9. HRMS(ESI) $\mathrm{m} / z$ Calcd for $\mathrm{C}_{28} \mathrm{H}_{21} \mathrm{~F}_{3} \mathrm{~N}[\mathrm{M}]^{+}$: 428.1626; found: 428.1631 . 


\section{Hydroaminoalkylation of 1-Chloro-4-(phenylethynyl)benzene with $\mathrm{N}$-benzylaniline (10)}

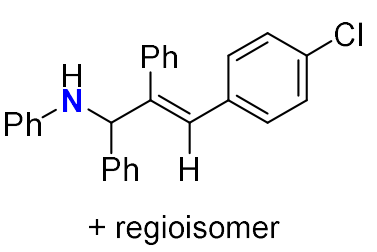

This reaction was carried out according to the general procedure $(48 \mathrm{~h}, 74 \%$ NMR yield, $1: 1.2,51 \%$ isolated yield). ${ }^{1} \mathrm{H}$ NMR $\left(\mathrm{CDCl}_{3}, 400 \mathrm{MHz}\right) \delta=7.37-$ $7.28(\mathrm{~m}, 11 \mathrm{H}), 7.24-7.13(\mathrm{~m}, 11 \mathrm{H}), 7.11-7.05(\mathrm{~m}, 4 \mathrm{H}), 7.00(\mathrm{~m}, 2 \mathrm{H}), 6.97-6.86$ $(\mathrm{m}, 4 \mathrm{H}), 6.82(\mathrm{~s}, 1 \mathrm{H}), 6.80(\mathrm{~s}, 1 \mathrm{H}), 6.74(\mathrm{~m}, 2 \mathrm{H}), 6.69(\mathrm{~m}, 4 \mathrm{H}), 5.15(\mathrm{~s}, 1 \mathrm{H})$, 5.13 (s, 1H), 4.24 (br s, 2H); ${ }^{13} \mathrm{C} \mathrm{NMR}\left(\mathrm{CDCl}_{3}, 100 \mathrm{MHz}\right) \delta=146.9,146.9,142.4,140.6,140.4,139.0$, $137.8,136.4$, 135.5, 133.4, 132.5, 130.7, 130.7, 129.5, 129.3, 129.3, 129.1, 128.9, 128.9, 128.8, 128.8, $128,1,128.0,128.0,127.9,127.7,127.1,126.9,118.3,118.3,114.0,114.0,66.5,66.3$. HRMS(ESI) $\mathrm{m} / z$ Calcd for $\mathrm{C}_{27} \mathrm{H}_{21} \mathrm{ClN}[\mathrm{M}]^{+}: 394.1363$; found: 394.1373 .

\section{Hydroaminoalkylation of 2-(Phenylethynyl)pyridine with $N$-benzylaniline (11)}

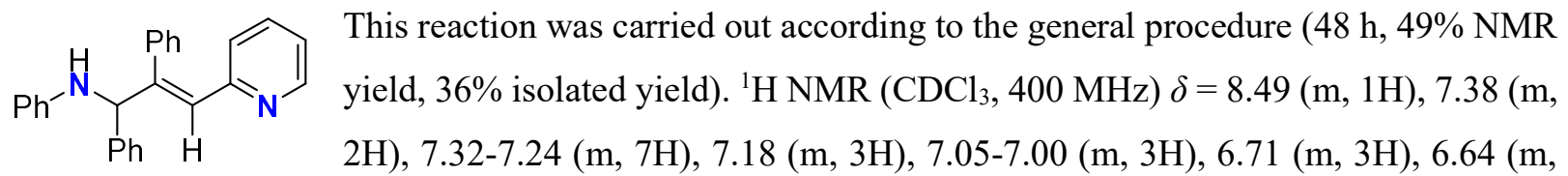

1H), $5.28(\mathrm{~d}, J=2.9 \mathrm{~Hz}, 1 \mathrm{H}), 4.28(\mathrm{~d}, J=2.9 \mathrm{~Hz}, 1 \mathrm{H}) ;{ }^{13} \mathrm{C} \mathrm{NMR}\left(\mathrm{CDCl}_{3}, 100 \mathrm{MHz}\right) \delta=154.8,146.9$, 146.6, 139.9, 138.2 137.8, 129.4, 129.0, 129.0, 128.7, 128.2, 128.0, 128.0, 124.5, 122.0, 118.1, 113.6, 66.0. HRMS(ESI) $m / z$ Calcd for $\mathrm{C}_{26} \mathrm{H}_{22} \mathrm{~N}_{2}[\mathrm{M}]^{+}: 362.1783$; found: 362.1789 .

\section{Hydroaminoalkylation of 1-Phenyl-1-propyne with $N$-benzylaniline (12) $)^{11}$}<smiles>C/C(=C\c1ccccc1)C(Nc1ccccc1)c1ccccc1</smiles>

+ regioisomer

This reaction was carried out according to the general procedure (48 h, 40\% NMR yield, 1:4.7, 40\% isolated yield). ${ }^{1} \mathrm{H} \mathrm{NMR}\left(\mathrm{CDCl}_{3}, 400 \mathrm{MHz}\right) \delta=7.45(\mathrm{~m}, 2 \mathrm{H}), 7.36$ $7.13(\mathrm{~m}, 10 \mathrm{H}), 6.98(\mathrm{~m}, 1 \mathrm{H}), 6.86-6.70(\mathrm{~m}, 4 \mathrm{H}), 6.04(\mathrm{br} \mathrm{s}, 1 \mathrm{H}), 5.07$ (s, 1H), $1.83(\mathrm{~s}$, $3 \mathrm{H}), 1.57$ (m, 3H). HRMS(ESI) $\mathrm{m} / z$ Calcd for $\mathrm{C}_{22} \mathrm{H}_{20} \mathrm{~N}[\mathrm{M}]^{+}:$298.1596; found:

298.1600.

\section{Hydroaminoalkylation of (Cyclohexylethynyl)benzene with $N$-benzylaniline (13)}

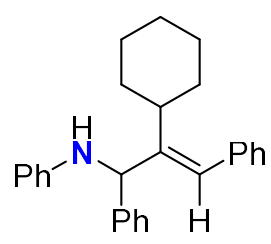

+ regioisomer
This reaction was carried out according to the general procedure (48 h, 76\% NMR yield, $1: 1.2,38 \%$ isolated yield). ${ }^{1} \mathrm{H} \mathrm{NMR}\left(\mathrm{CDCl}_{3}, 400 \mathrm{MHz}\right) \delta=7.43(\mathrm{~m}, 2 \mathrm{H}), 7.36-$ $7.20(\mathrm{~m}, 16 \mathrm{H}), 7.15(\mathrm{~m}, 6 \mathrm{H}), 6.93(\mathrm{~m}, 2 \mathrm{H}), 6.72(\mathrm{~m}, 2 \mathrm{H}), 6.61(\mathrm{~m}, 3 \mathrm{H}), 6.43$ (br s, 1H), $5.69(\mathrm{~m}, 1 \mathrm{H}), 5.09(\mathrm{~s}, 1 \mathrm{H}), 5.06(\mathrm{~s}, 1 \mathrm{H}), 4.02(\mathrm{br} \mathrm{s}, 1 \mathrm{H}), 2.87(\mathrm{~m}, 1 \mathrm{H}), 1.95(\mathrm{~m}, 1 \mathrm{H})$, 1.85-0.94 (m, 20H); ${ }^{13} \mathrm{C} \mathrm{NMR}\left(\mathrm{CDCl}_{3}, 100 \mathrm{MHz}\right) \delta=148.2,147.2,139.3,137.9,136.4$, 59.3, 40.3, 37.6, 33.3, 33.1, 32.8, 31.7, 26.5, 26.5, 26.0, 25.7, 25.6. HRMS(ESI) $m / z$ Calcd for $\mathrm{C}_{27} \mathrm{H}_{28} \mathrm{~N}$ $[\mathrm{M}]^{+}:$366.2222; found: 366.2226 . 


\section{Hydroaminoalkylation of 1-Phenyl-2-trimethylsilylacetylene with $N$-benzylaniline (14)}

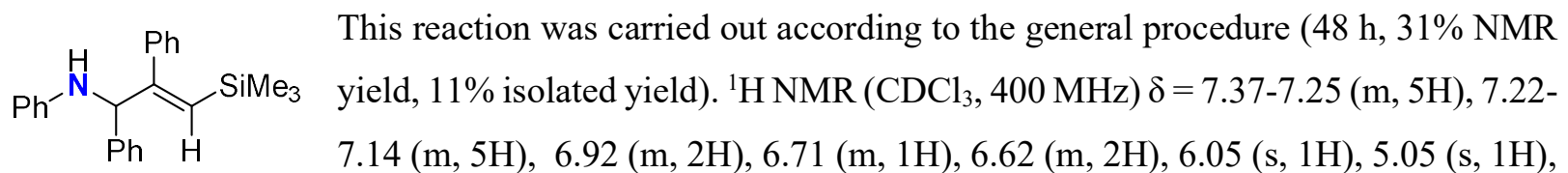

$4.12($ br s, $1 \mathrm{H}),-0.22(\mathrm{~s}, 9 \mathrm{H})) ;{ }^{13} \mathrm{C} \mathrm{NMR}\left(\mathrm{CDCl}_{3}, 100 \mathrm{MHz}\right) \delta=157.0,147.4,141.75,129.19,129.01$, $128.72,128.67,127.94,127.80,127.78,127.59,127.37,117.7,113.7,67.3,0.0$. HRMS(ESI) $\mathrm{m} / z$ Calcd for $\mathrm{C}_{24} \mathrm{H}_{26} \mathrm{NSi}[\mathrm{M}]^{+}:$356.1835; found: 356.1838 .

\section{Hydroaminoalkylation of Diphenylacetylene with $N$-benzyl-4-methylaniline (15)}

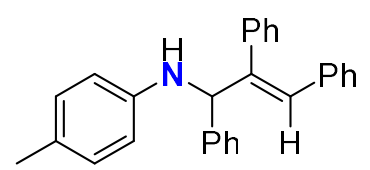

This reaction was carried out according to the general procedure ( $24 \mathrm{~h}, 84 \%$

NMR yield, $69 \%$ isolated yield). ${ }^{1} \mathrm{H}$ NMR $\left(\mathrm{CDCl}_{3}, 300 \mathrm{MHz}\right) \delta=7.41(\mathrm{~m}, 2 \mathrm{H})$, 7.38-7.30 (m, 3H), 7.30-7.23 (m, 3H), 7.12-7.05 (m, 3H), 7.05-6.92 (m, 4H), 6.96-6.90 (m, 3H), $6.68(\mathrm{~m}, 2 \mathrm{H}), 5.18(\mathrm{~s}, 1 \mathrm{H}), 4.33(\mathrm{br} \mathrm{s}, 1 \mathrm{H}), 2.27(\mathrm{~s}, 3 \mathrm{H}) ;{ }^{13} \mathrm{C} \mathrm{NMR}\left(\mathrm{CDCl}_{3}, 75 \mathrm{MHz}\right) \delta$ $=144.6,141.8,140.9,139.4,136.7,133.0,129.8,129.4,129.2,128.7,128.7,128.3,128.0,128.0,127.7$, 127.4, 126.8, 114.2, 66.8, 20.6. HRMS(ESI) $\mathrm{m} / z$ Calcd for $\mathrm{C}_{28} \mathrm{H}_{24} \mathrm{~N}[\mathrm{M}]^{+}: 374.1909$; found: 374.1914.

\section{Hydroaminoalkylation of Diphenylacetylene with $N$-benzyl-4-chloroaniline (16)}<smiles>Clc1ccc(NC(C=C(c2ccccc2)c2ccccc2)c2ccccc2)cc1</smiles>

This reaction was carried out according to the general procedure (48 h, $42 \%$ NMR yield, 25\% isolated yield). ${ }^{1} \mathrm{H}$ NMR $\left(\mathrm{CDCl}_{3}, 400 \mathrm{MHz}\right) \delta=7.43-7.32$ (m, 5H), 7.30-7.23 (m, 3H), 7.17-7.06 (m, 5H), $6.96(\mathrm{~m}, 4 \mathrm{H}), 6.85(\mathrm{~s}, 1 \mathrm{H}), 6.62$ (m, 2H), $5.18(\mathrm{~s}, 1 \mathrm{H}), 4.24$ (br s, $1 \mathrm{H}) ;{ }^{13} \mathrm{C} \mathrm{NMR}\left(\mathrm{CDCl}_{3}, 100 \mathrm{MHz}\right) \delta=145.6,141.3,140.6,139.1,136.5$, 129.4, 129.2, 129.2, 128.9, 128.8, 128.6, 128.1, 128.0, 127.6, 127.0, 122.6, 115.0, 66.3. HRMS(FD) $\mathrm{m} / \mathrm{z}$ Calcd for $\mathrm{C}_{27} \mathrm{H}_{22} \mathrm{ClN}[\mathrm{M}]^{+}: 395.14408$; found: 395.14280 .

\section{Hydroaminoalkylation of Diphenylacetylene with $N$-benzyl-4-fluoroaniline (17)}<smiles>Fc1ccc(NC(/C(=C/c2ccccc2)c2ccccc2)c2ccccc2)cc1</smiles>

This reaction was carried out according to the general procedure $(48 \mathrm{~h}, 78 \%$ NMR yield, 66\% isolated yield). ${ }^{1} \mathrm{H}$ NMR $\left(\mathrm{CDCl}_{3}, 400 \mathrm{MHz}\right) \delta=7.37(\mathrm{~m}, 2 \mathrm{H})$, 7.33-7.26 (m, 3H), $7.20(\mathrm{~m}, 3 \mathrm{H}), 7.05(\mathrm{~m}, 3 \mathrm{H}), 6.95-6.83(\mathrm{~m}, 7 \mathrm{H}), 6.59$ (br m, 2H), $5.11(\mathrm{~s}, 1 \mathrm{H}), 4.08(\mathrm{br} \mathrm{s}, 1 \mathrm{H}) ;{ }^{13} \mathrm{C} \mathrm{NMR}\left(\mathrm{CDCl}_{3}, 100 \mathrm{MHz}\right) \delta=156.2(\mathrm{~d}, J=235.4 \mathrm{~Hz}), 143.4,141.6$, $140.8,139.2,136.6,129.4,129.2,128.8,128.7,128.4,128.0,128.0,127.9,127.6,127.0,115.8(\mathrm{~d}, J=22.3$ $\mathrm{Hz}), 114.7,66.9 ;{ }^{19} \mathrm{~F}$ NMR $\left(\mathrm{CDCl}_{3}, 282 \mathrm{MHz}\right) \delta=127.9$. HRMS(ESI) $m / z$ Calcd for $\mathrm{C}_{27} \mathrm{H}_{21} \mathrm{FN}[\mathrm{M}]^{+}$: 378.1658; found: 378.1663 . 


\section{Hydroaminoalkylation of Diphenylacetylene with $N$-(4-methylbenzyl)aniline (18)}

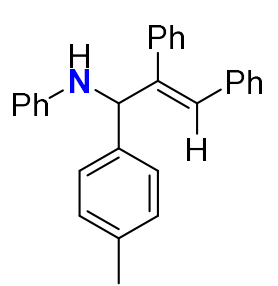

This reaction was carried out according to the general procedure $(24 \mathrm{~h}, 41 \%$ NMR yield; $48 \mathrm{~h}, 72 \%$ NMR yield, 56\% isolated yield). ${ }^{1} \mathrm{H}$ NMR $\left(\mathrm{CDCl}_{3}, 400 \mathrm{MHz}\right) \delta=$ 7.28-7.21 (m, 5H), $7.16(\mathrm{~m}, 2 \mathrm{H}), 7.12(\mathrm{~m}, 2 \mathrm{H}), 7.04(\mathrm{~m}, 3 \mathrm{H}), 6.97(\mathrm{~m}, 2 \mathrm{H}), 6.91(\mathrm{~m}$, $3 \mathrm{H}), 6.72(\mathrm{~m}, 3 \mathrm{H}), 5.12(\mathrm{~s}, 1 \mathrm{H}), 4.22(\mathrm{br} \mathrm{s}, 1 \mathrm{H}), 2.33(\mathrm{~s}, 3 \mathrm{H}) ;{ }^{13} \mathrm{C} \mathrm{NMR}\left(\mathrm{CDCl}_{3}, 100\right.$ MHz) $\delta=146.5,139.6,139.5,137.6,136.7,136.7,133.7,129.5,129.3,129.2$ 128.7, $128.7,128.0,128.0,127.4,126.8,118.5,114.5,66.7,21.3$. HRMS(ESI) $m / z$ Calcd for $\mathrm{C}_{28} \mathrm{H}_{24} \mathrm{~N}[\mathrm{M}]^{+}$: 374.1909; found: 374.1911 .

\section{Hydroaminoalkylation of Diphenylacetylene with $N$-(4-chlorobenzyl)aniline (19)}<smiles>Clc1ccc(C(=Cc2ccccc2)C(c2ccccc2)c2ccccc2)cc1</smiles>

This reaction was carried out according to the general procedure $(24 \mathrm{~h}, 79 \%$ NMR yield, $40 \%$ isolated yield). ${ }^{1} \mathrm{H} \mathrm{NMR}\left(\mathrm{CDCl}_{3}, 400 \mathrm{MHz}\right) \delta=7.40-7.28(\mathrm{~m}, 7 \mathrm{H}), 7.22$ (m, 2H), $7.12(\mathrm{~m}, 3 \mathrm{H}), 7.02(\mathrm{~m}, 2 \mathrm{H}), 6.96(\mathrm{~m}, 2 \mathrm{H}), 6.89(\mathrm{~s}, 1 \mathrm{H}), 6.80(\mathrm{~m}, 1 \mathrm{H}) 6.72(\mathrm{~m}$, 2H), $5.23(\mathrm{~s}, 1 \mathrm{H}), 4.45$ (br s, $1 \mathrm{H}) ;{ }^{13} \mathrm{C} \mathrm{NMR}\left(\mathrm{CDCl}_{3}, 100 \mathrm{MHz}\right) \delta=146.6,141.4,139.5$, 139.0, 136.4, 133.5, 130.2, 129.4, 129.4, 129.3, 129.2, 129.0, 128.8, 128.1, 127.7, 127.1, 118.5, 114.2, 65.9. HRMS(ESI) $m / z$ Calcd for $\mathrm{C}_{27} \mathrm{H}_{21} \mathrm{CIN}[\mathrm{M}]^{+}: 394.1363$; found: 394.1365 .

\section{Catalytic Hydroaminoalkylation of Diphenylacetylene with $N$-(4-fluorobenzyl)aniline (20)}

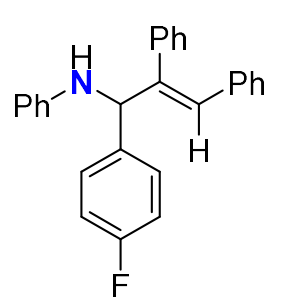

This reaction was carried out according to the general procedure $(24 \mathrm{~h}, 80 \%$ NMR yield, 61\% isolated yield). ${ }^{1} \mathrm{H}$ NMR $\left(\mathrm{CDCl}_{3}, 400 \mathrm{MHz}\right) \delta=7.41(\mathrm{~m}, 2 \mathrm{H}), 7.30(\mathrm{~m}$, 3H), $7.22(\mathrm{~m}, 2 \mathrm{H}), 7.12(\mathrm{~m}, 3 \mathrm{H}), 7.09-7.00(\mathrm{~m}, 4 \mathrm{H}), 6.97$ (m, 2H), $6.88(\mathrm{~s}, 1 \mathrm{H}), 6.79$ $(\mathrm{m}, 1 \mathrm{H}), 6.72(\mathrm{~m}, 2 \mathrm{H}), 5.23(\mathrm{~s}, 1 \mathrm{H}), 4.19(\mathrm{br} \mathrm{s}, 1 \mathrm{H}) ;{ }^{13} \mathrm{C} \mathrm{NMR}\left(\mathrm{CDCl}_{3}, 100 \mathrm{MHz}\right) \delta=$ $162.4(\mathrm{~d}, J=245.9 \mathrm{~Hz}), 147.0,141.6,139.2,136.8,136.5,129.6(\mathrm{~d}, J=8.3 \mathrm{~Hz}), 129.4$, 129.4, 129.2, 128.8, 128.5, 128.1, 127.6, 127.0, 118.2, 115.6 (d, $J=21.5 \mathrm{~Hz}), 113.9,65.6 ;{ }^{19} \mathrm{~F} \mathrm{NMR}\left(\mathrm{CDCl}_{3}\right.$, $282 \mathrm{MHz}) \delta=115.3$. HRMS(ESI) $\mathrm{m} / z$ Calcd for $\mathrm{C}_{27} \mathrm{H}_{21} \mathrm{FN}[\mathrm{M}]^{+}:$378.1658; found: 378.1664. 


\section{Proposed General Catalytic Cycle for Hydroaminoalkylation of Alkynes}

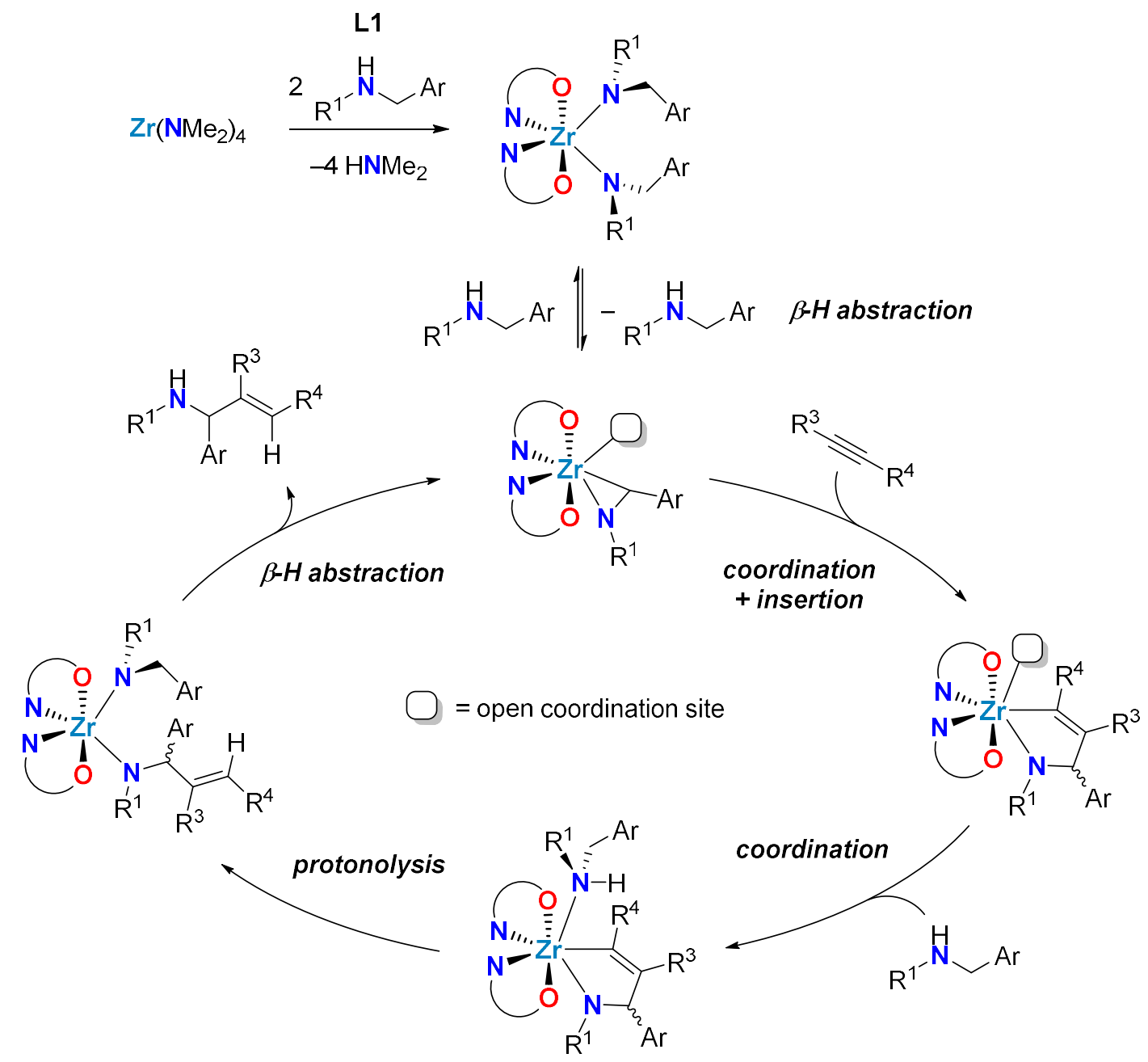




\section{NMR Spectra}

Figure S1. ${ }^{1} \mathrm{H}$ NMR spectrum of 1 (C6 $\left.\mathrm{D}_{6}, 400 \mathrm{MHz}, 298 \mathrm{~K}\right)$.

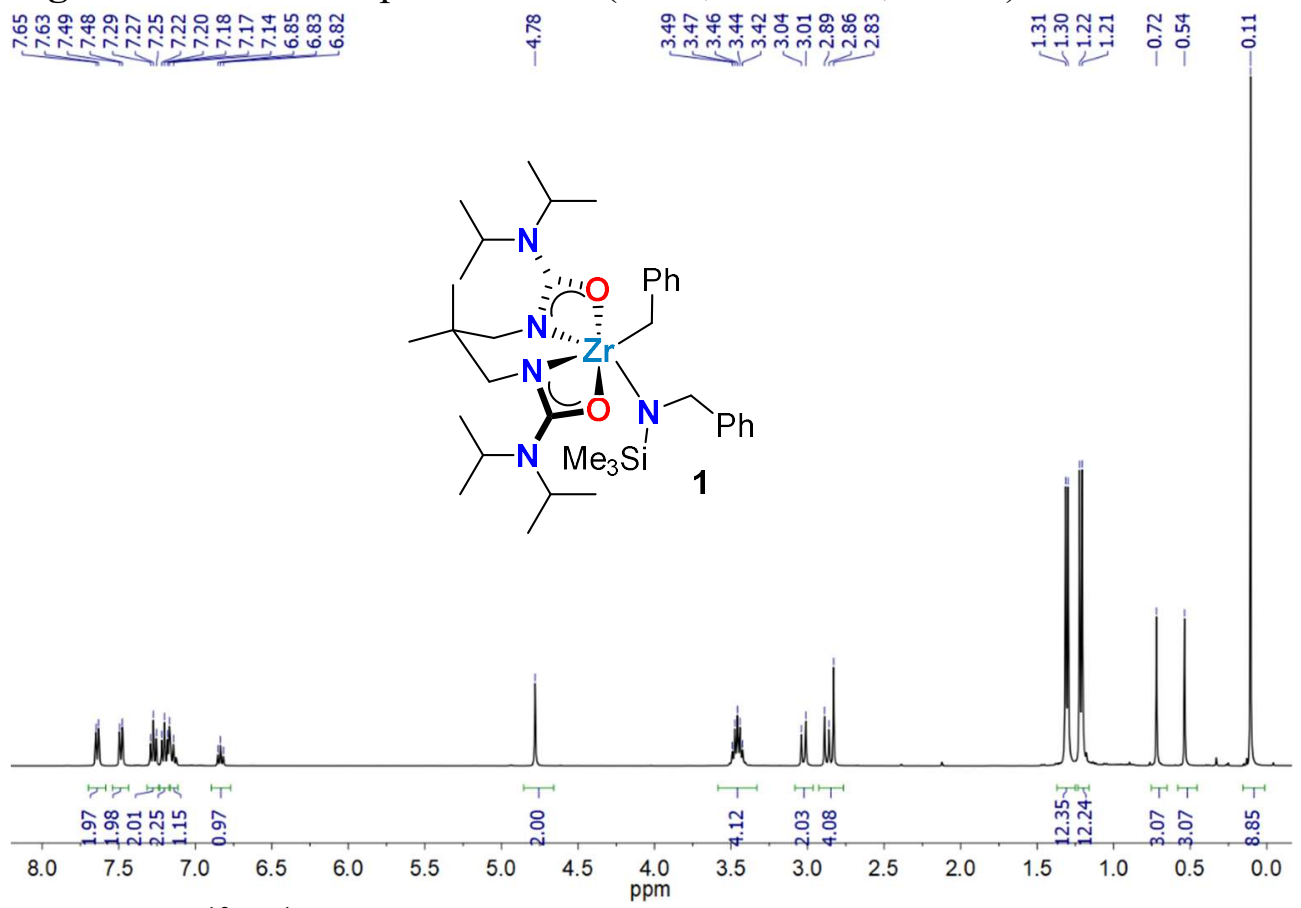

Figure S2. ${ }^{13} \mathrm{C}\left\{{ }^{1} \mathrm{H}\right\}$ NMR spectrum of $1\left(\mathrm{C}_{6} \mathrm{D}_{6}, 100 \mathrm{MHz}, 298 \mathrm{~K}\right)$.

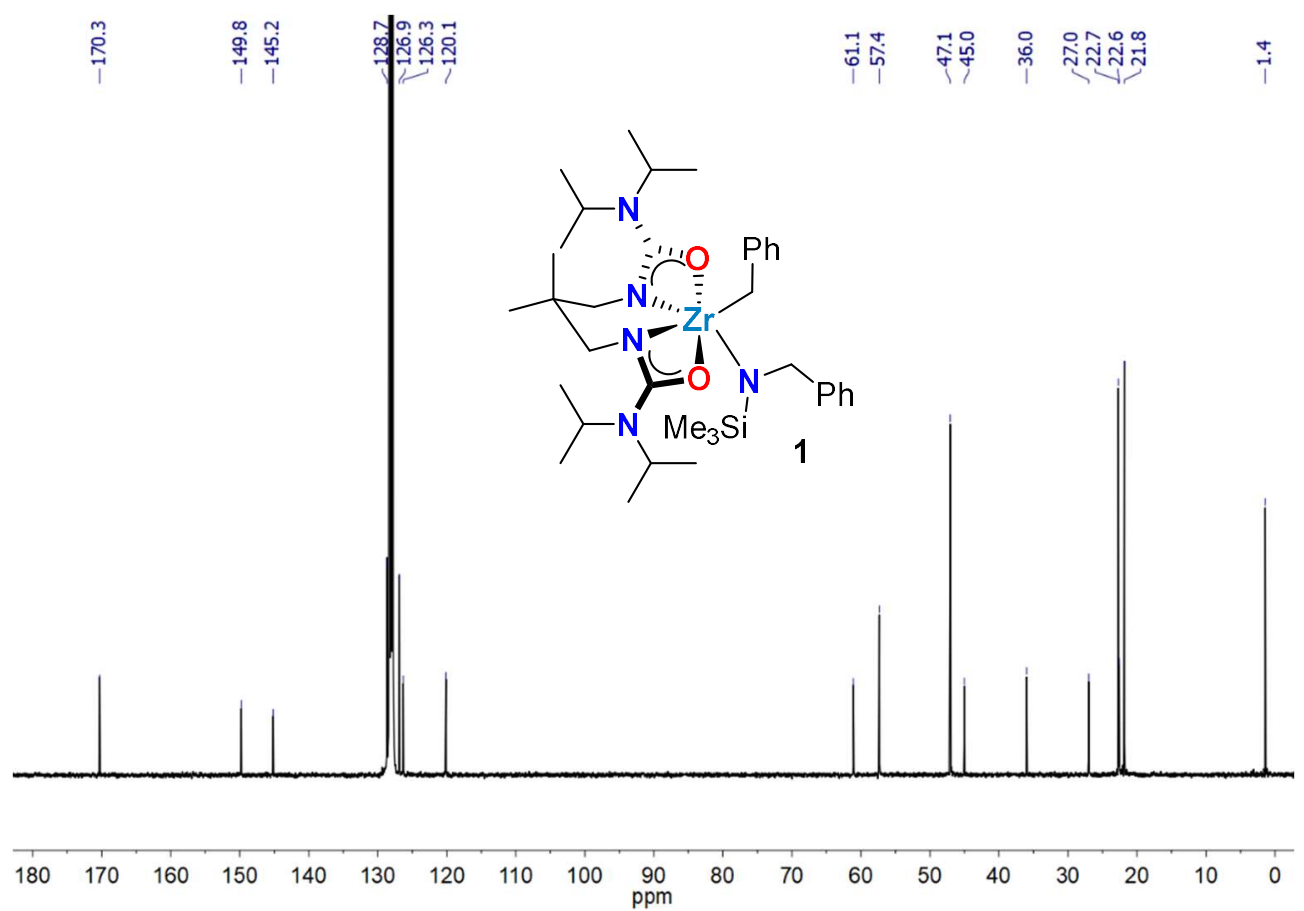


Figure S3. ${ }^{1} \mathrm{H}$ NMR spectrum of $2\left(\mathrm{C}_{6} \mathrm{D}_{6}, 400 \mathrm{MHz}, 298 \mathrm{~K}\right)$.

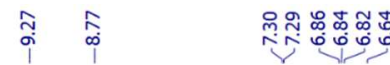
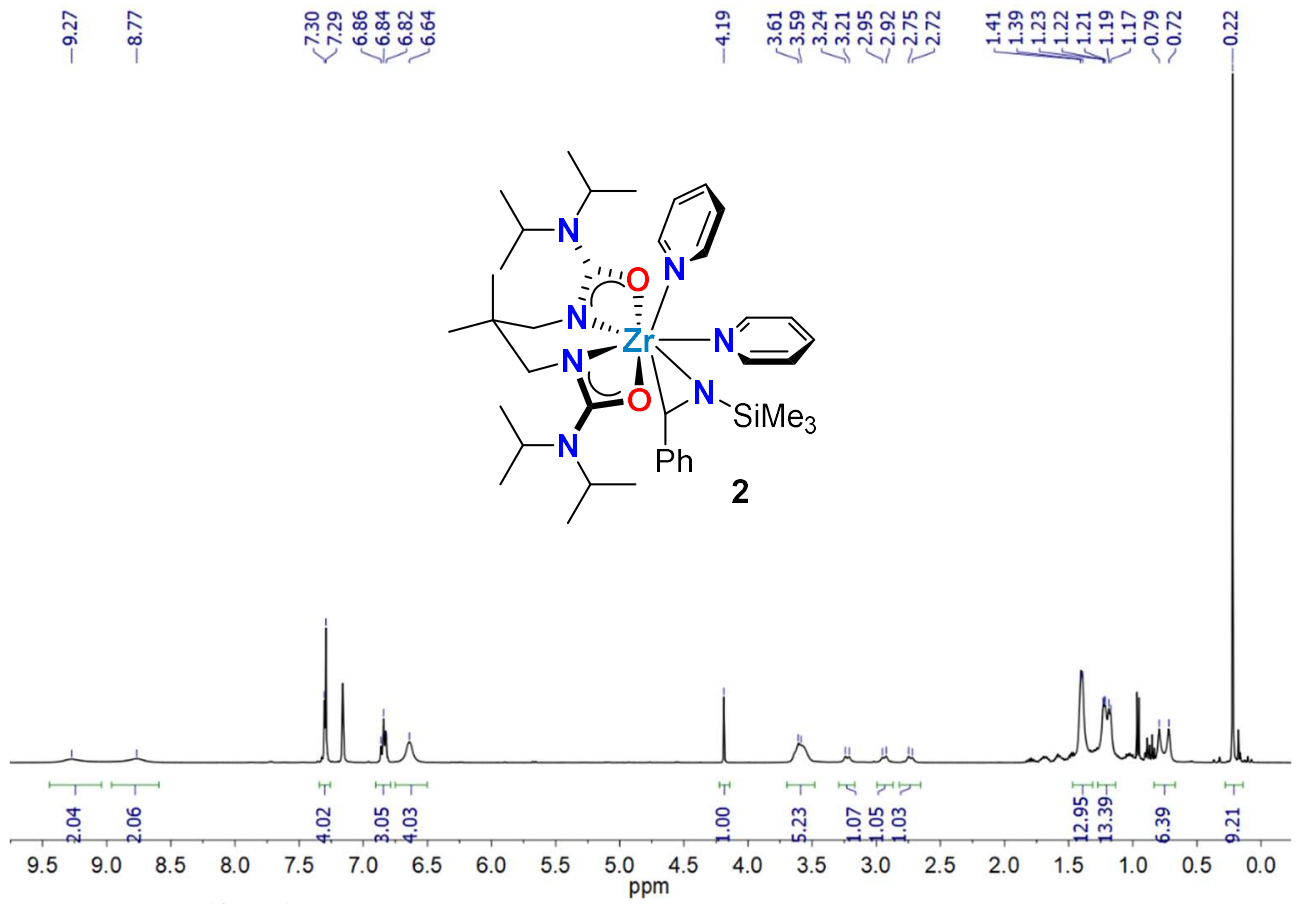

Figure S4. ${ }^{13} \mathrm{C}\left\{{ }^{1} \mathrm{H}\right\}$ NMR spectrum of $2\left(\mathrm{C}_{6} \mathrm{D}_{6}, 100 \mathrm{MHz}, 298 \mathrm{~K}\right)$.

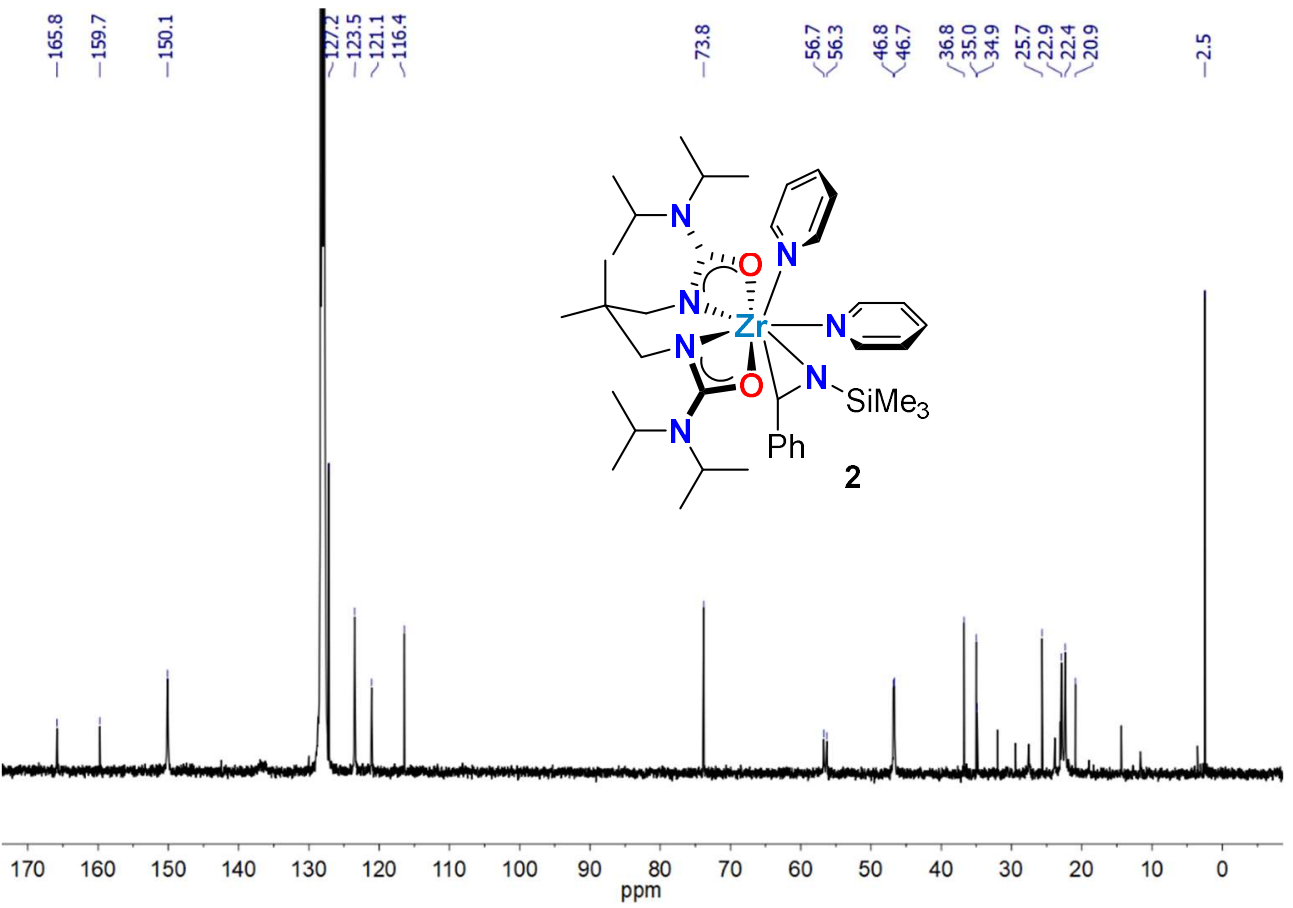


Figure S5. ${ }^{1} \mathrm{H}$ NMR spectrum of $3\left(\mathrm{C}_{6} \mathrm{D}_{6}, 300 \mathrm{MHz}, 298 \mathrm{~K}\right)$.

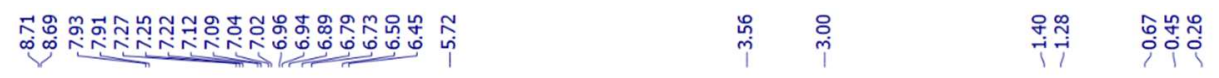
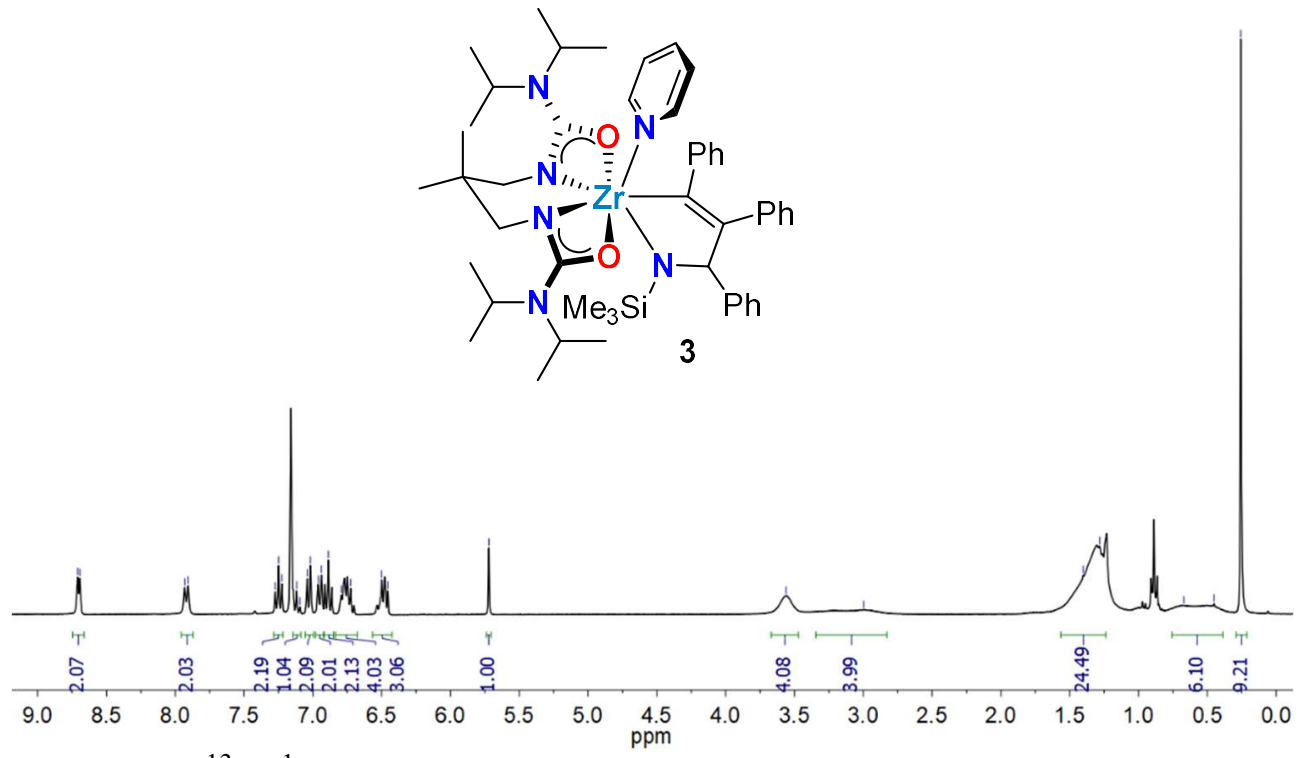

Figure S6. ${ }^{13} \mathrm{C}\left\{{ }^{1} \mathrm{H}\right\}$ NMR spectrum of $3\left(\mathrm{C}_{6} \mathrm{D}_{6}, 100 \mathrm{MHz}, 298 \mathrm{~K}\right)$.

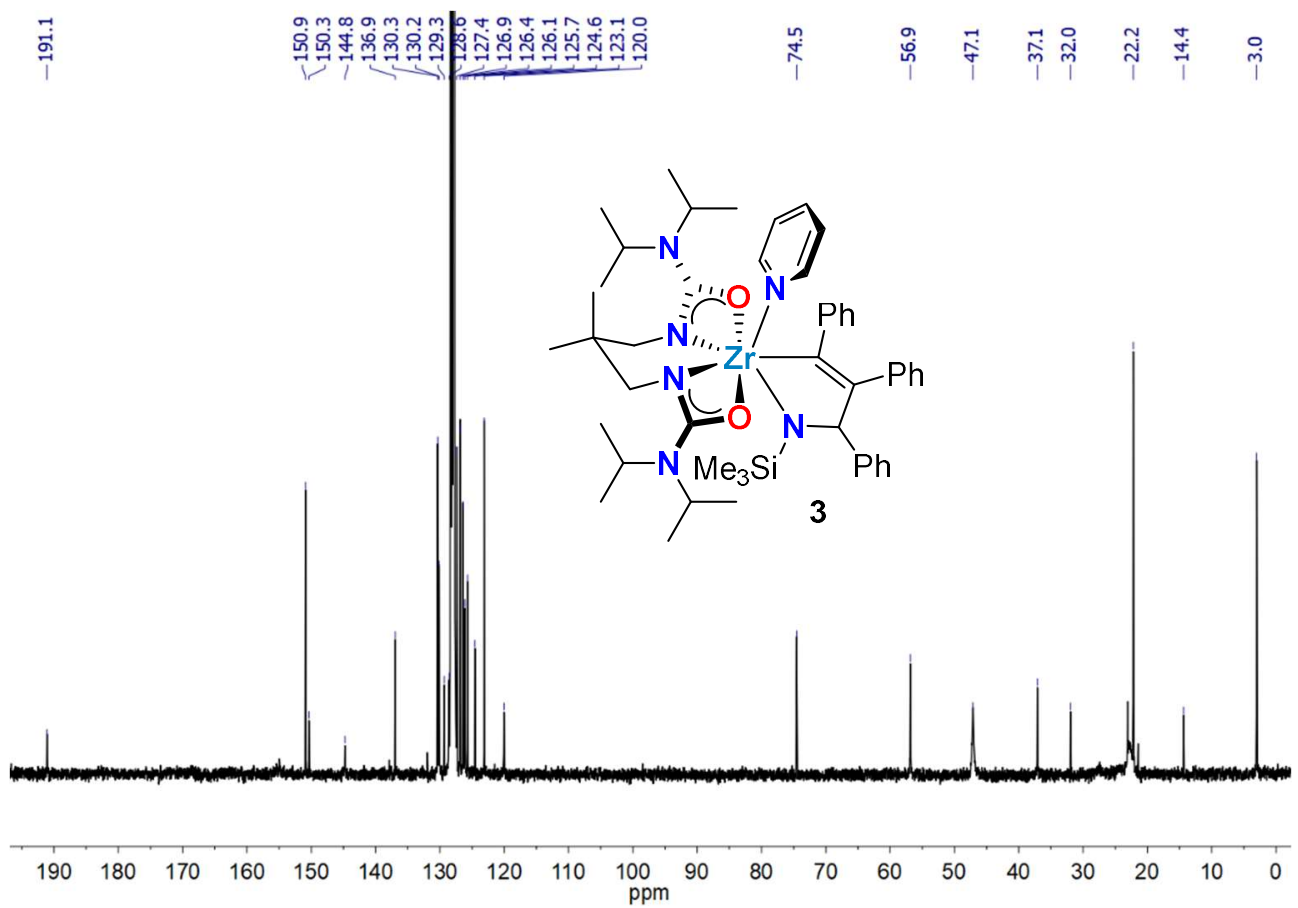


Figure S7. ${ }^{1} \mathrm{H}$ NMR spectrum of 5a in situ $\left(\mathrm{C}_{6} \mathrm{D}_{6}, 400 \mathrm{MHz}, 298 \mathrm{~K}\right)$.

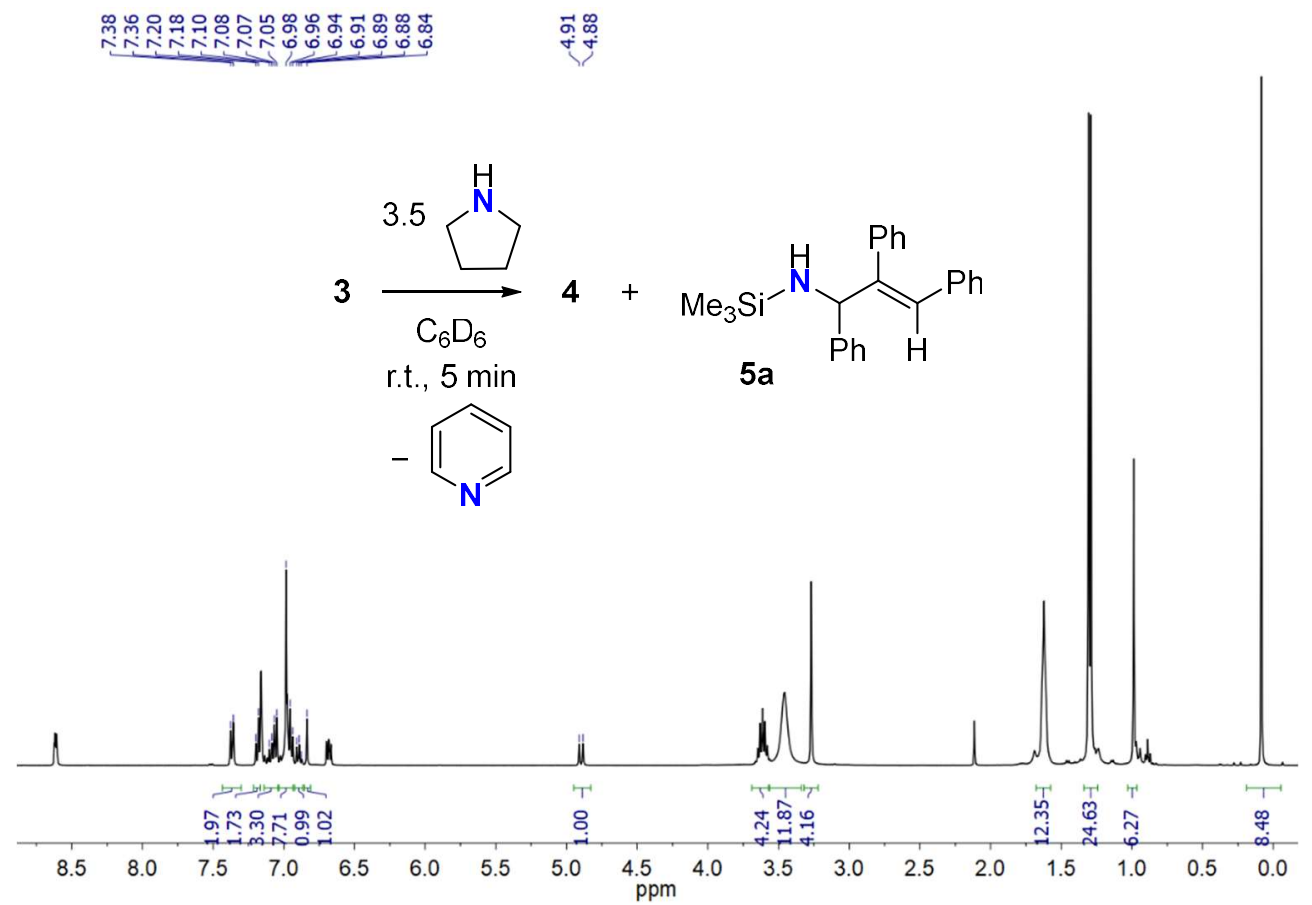

Figure S8. ${ }^{13} \mathrm{C}\left\{{ }^{1} \mathrm{H}\right\}$ NMR spectrum of 5a in situ $\left(\mathrm{C}_{6} \mathrm{D}_{6}, 100 \mathrm{MHz}, 298 \mathrm{~K}\right)$.

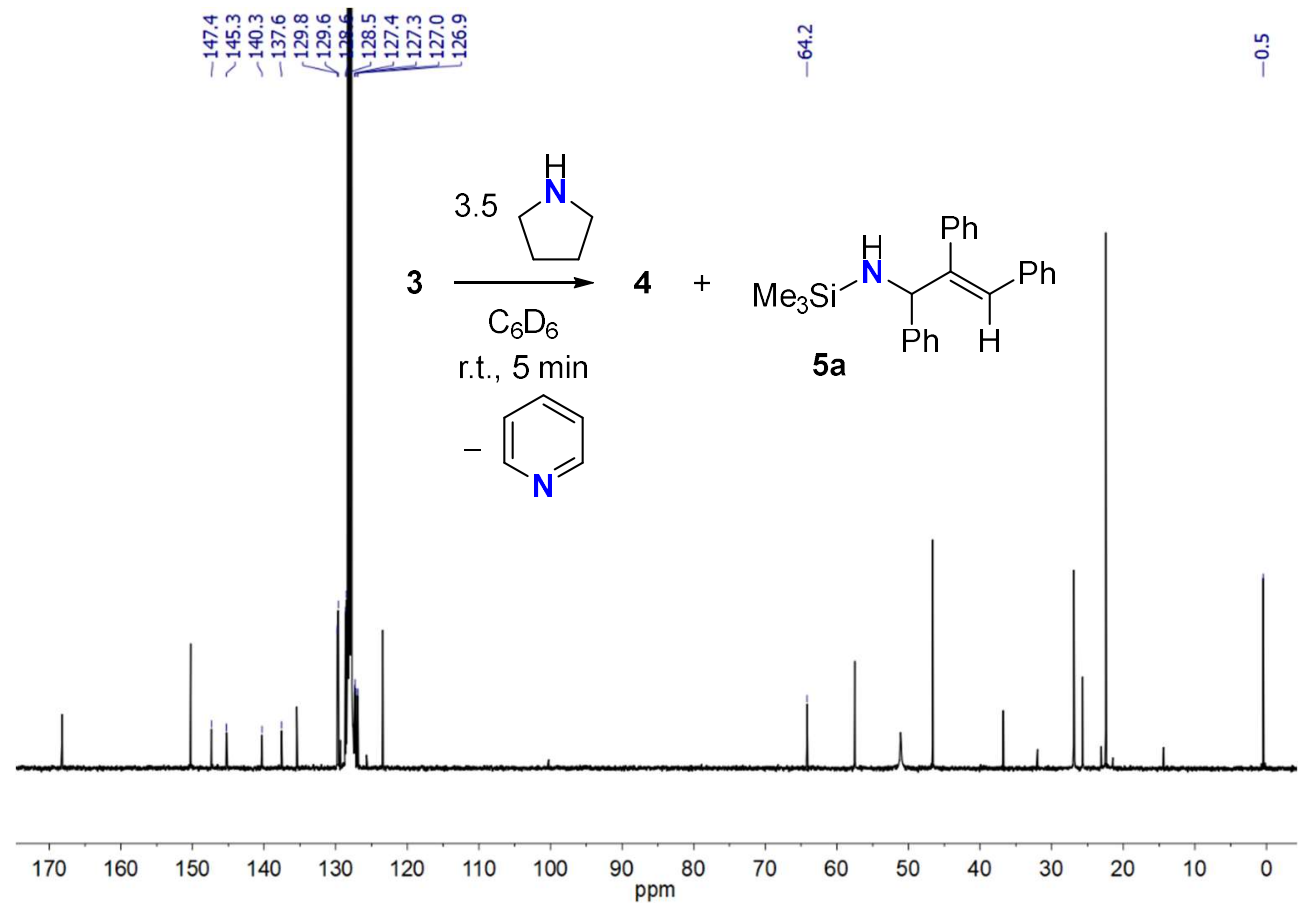


Figure S9. ${ }^{1} \mathrm{H} \mathrm{NMR}$ spectrum of $\mathbf{6 a}\left(\mathrm{CDCl}_{3}, 400 \mathrm{MHz}, 298 \mathrm{~K}\right)$.

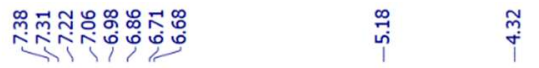

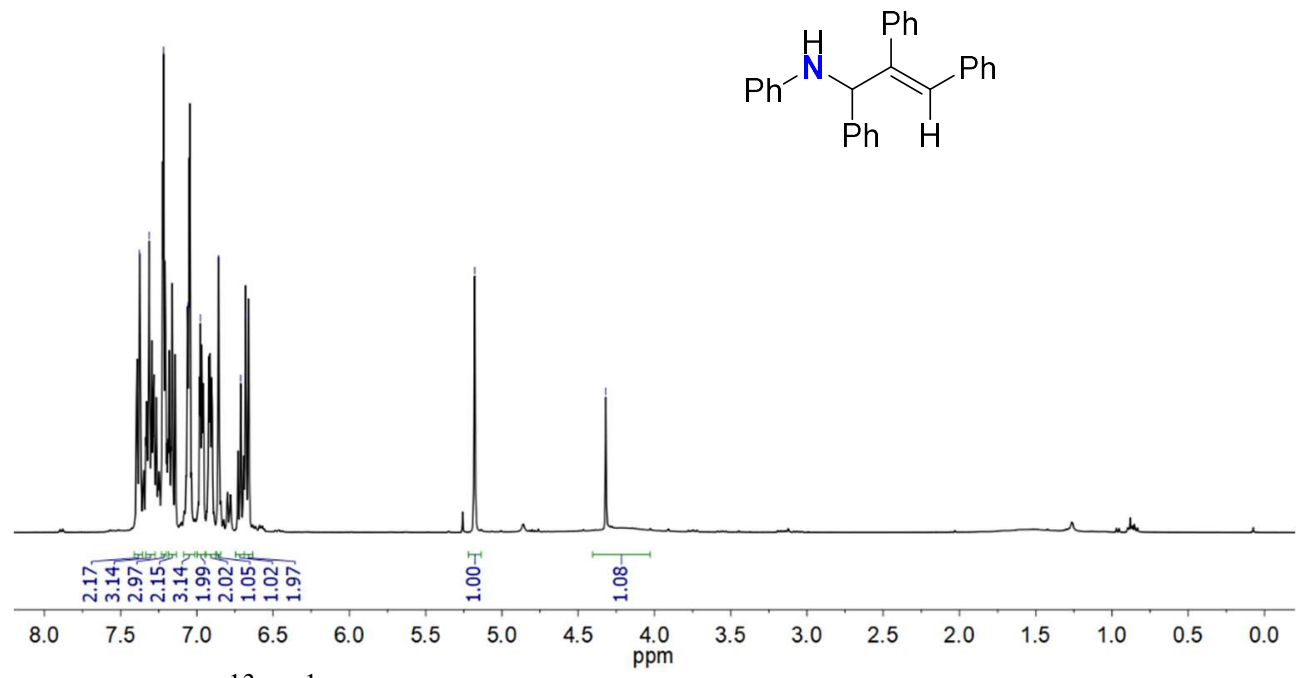

Figure S10. ${ }^{13} \mathrm{C}\left\{{ }^{1} \mathrm{H}\right\}$ NMR spectrum of $\mathbf{6 a}\left(\mathrm{CDCl}_{3}, 100 \mathrm{MHz}, 298 \mathrm{~K}\right)$.

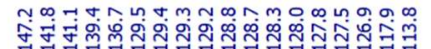

กั̊ำ

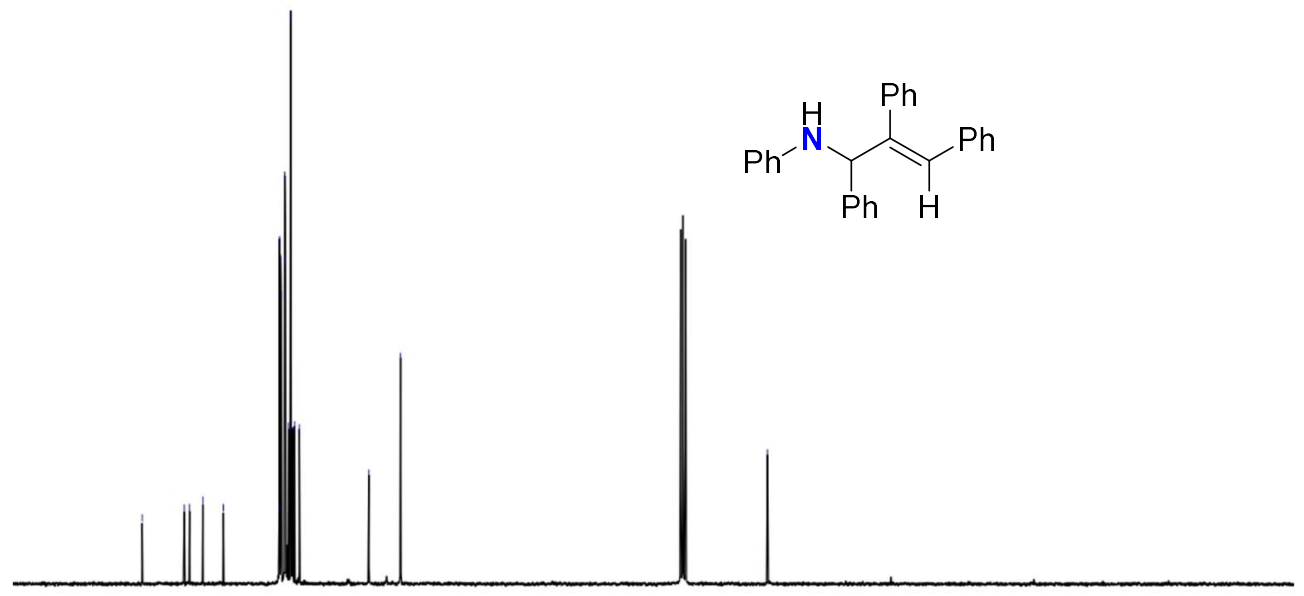

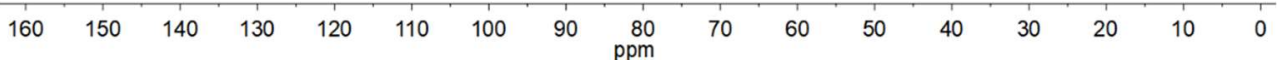


Figure S11. ${ }^{1} \mathrm{H}$ NMR spectrum of $\mathbf{7 a}$ and $\mathbf{7 b}\left(\mathrm{CDCl}_{3}, 400 \mathrm{MHz}, 298 \mathrm{~K}\right)$.

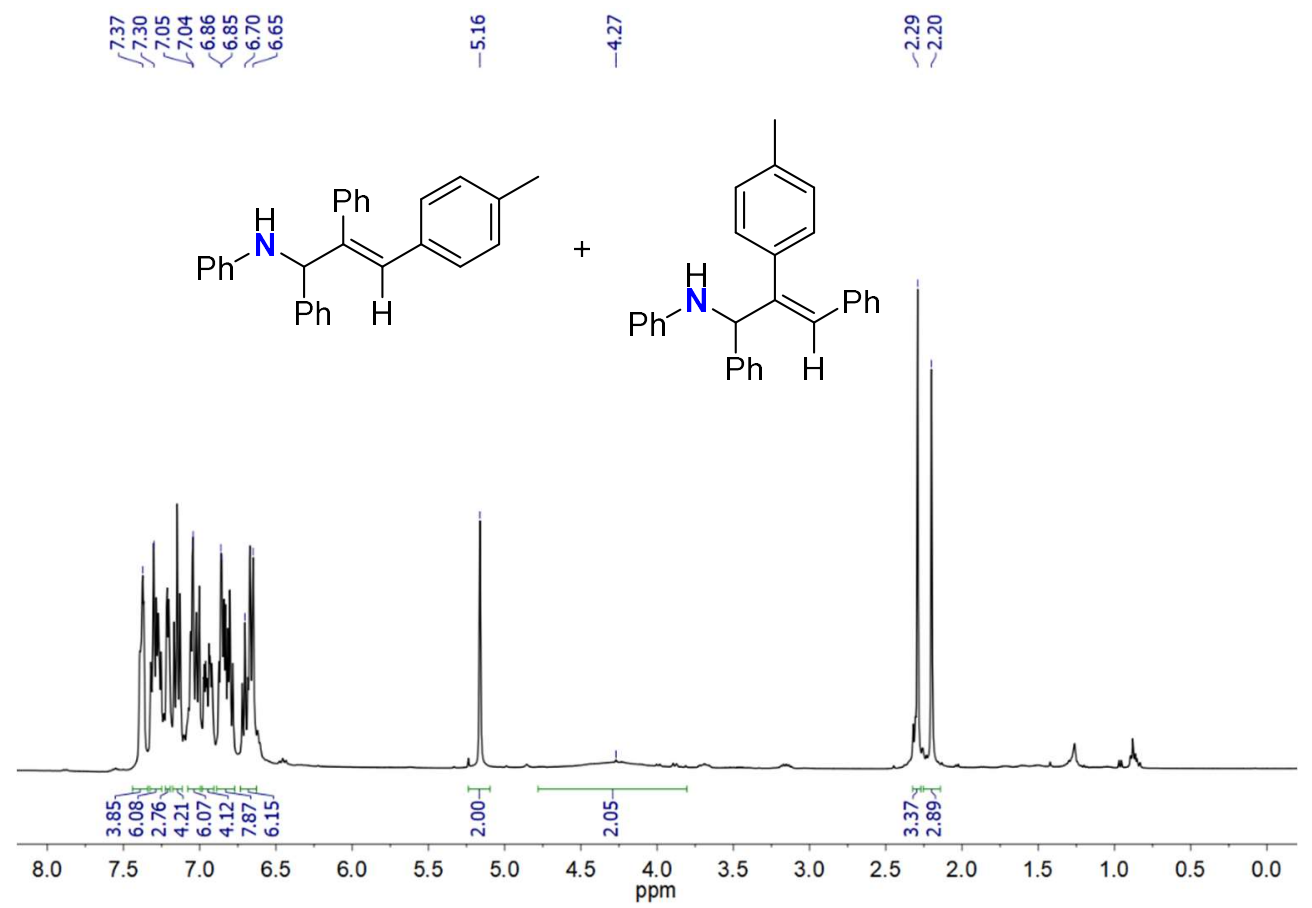

Figure S12. ${ }^{13} \mathrm{C}\left\{{ }^{1} \mathrm{H}\right\}$ NMR spectrum of $7 \mathbf{a}$ and $7 \mathbf{b}\left(\mathrm{CDCl}_{3}, 100 \mathrm{MHz}, 298 \mathrm{~K}\right)$.

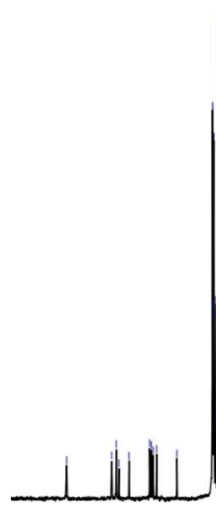<smiles>Cc1ccc(/C=C(\c2ccccc2)C(C=C(c2ccccc2)c2ccccc2)/C(=C/c2ccccc2)Nc2ccccc2)cc1</smiles>

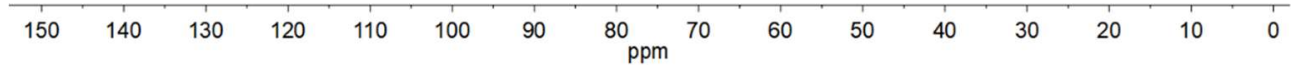


Figure S13. ${ }^{1} \mathrm{H}$ NMR spectrum of $\mathbf{8 a}$ and $\mathbf{8 b}\left(\mathrm{CDCl}_{3}, 400 \mathrm{MHz}, 298 \mathrm{~K}\right)$.

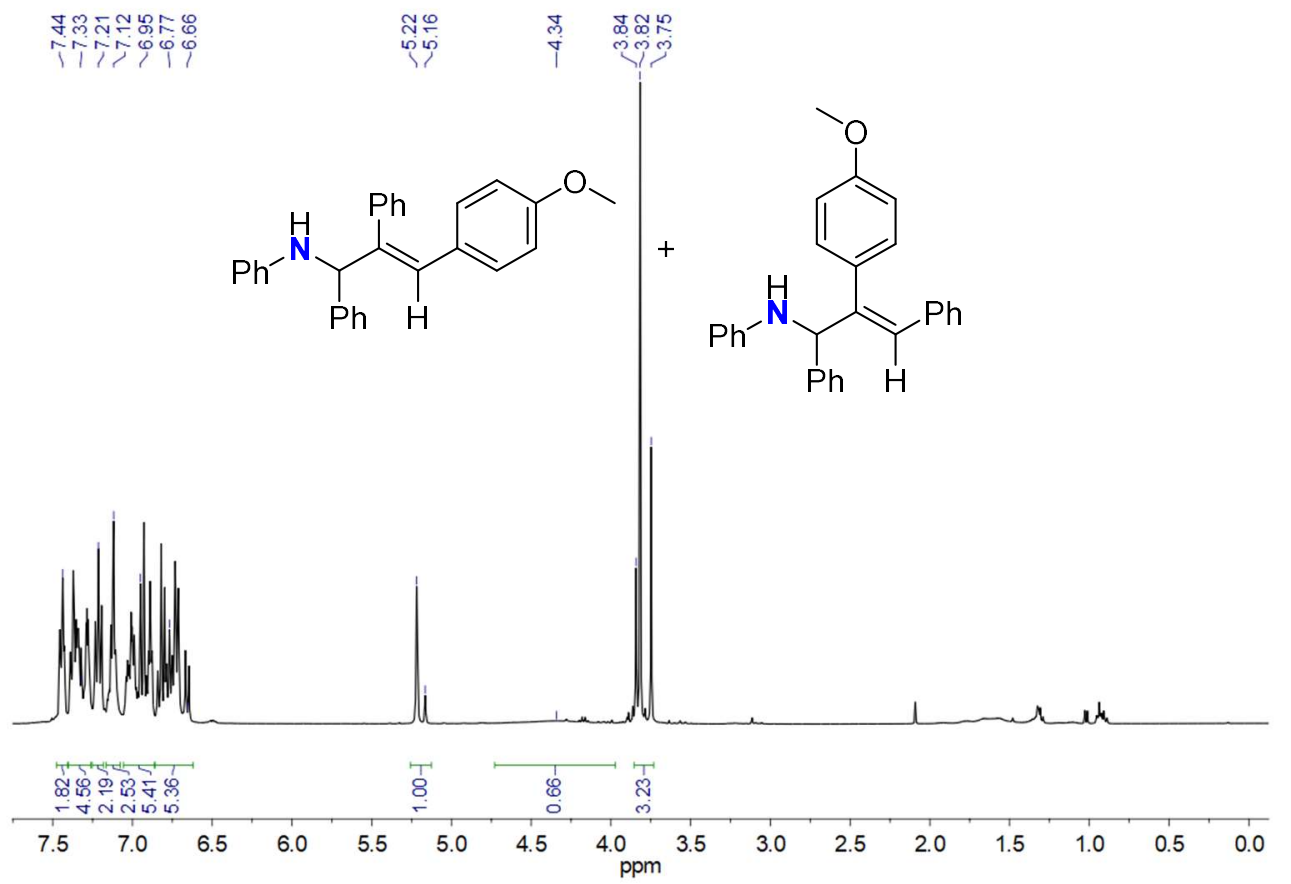

Figure S14. ${ }^{13} \mathrm{C}\left\{{ }^{1} \mathrm{H}\right\} \mathrm{NMR}$ spectrum of $\mathbf{8 a}$ and $\mathbf{8 b}\left(\mathrm{CDCl}_{3}, 100 \mathrm{MHz}, 298 \mathrm{~K}\right)$.
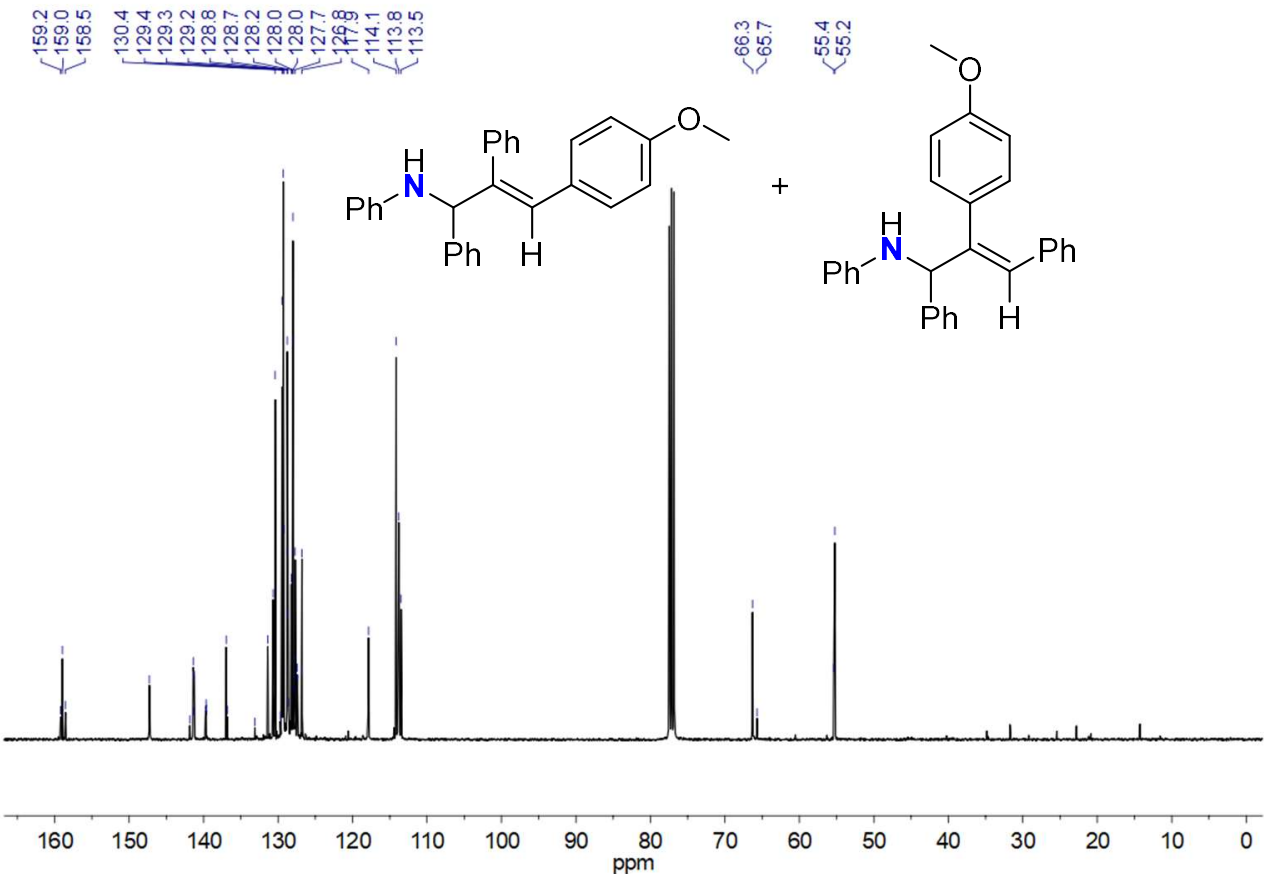
Figure S15. ${ }^{1} \mathrm{H}$ NMR spectrum of $9 \mathbf{a}$ and $9 \mathbf{b}\left(\mathrm{CDCl}_{3}, 300 \mathrm{MHz}, 298 \mathrm{~K}\right)$.

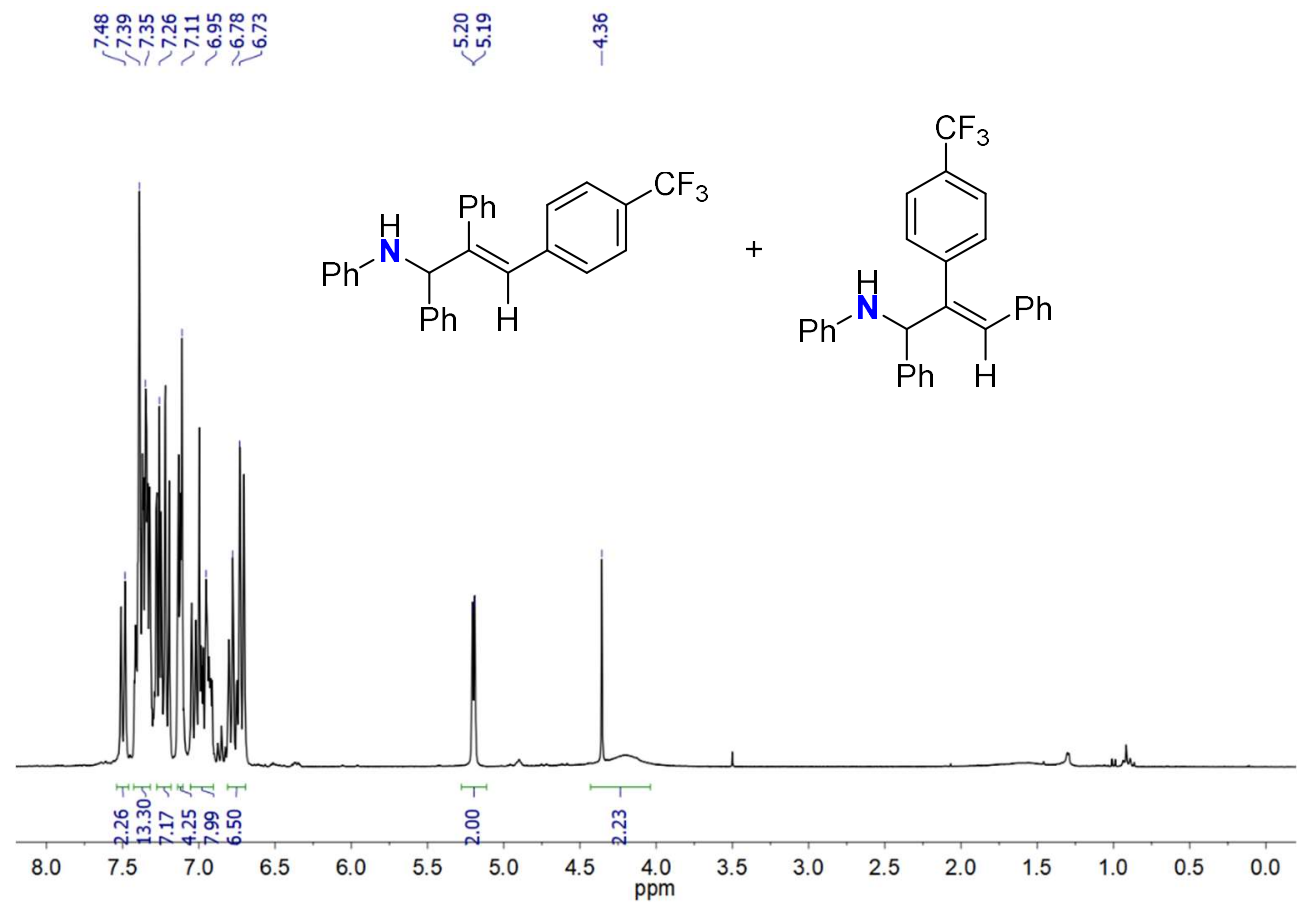

Figure S16. ${ }^{13} \mathrm{C}\left\{{ }^{1} \mathrm{H}\right\}$ NMR spectrum of $\mathbf{9 a}$ and $\mathbf{9 b}\left(\mathrm{CDCl}_{3}, 100 \mathrm{MHz}, 298 \mathrm{~K}\right)$.

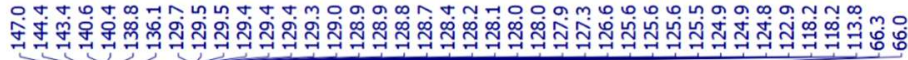
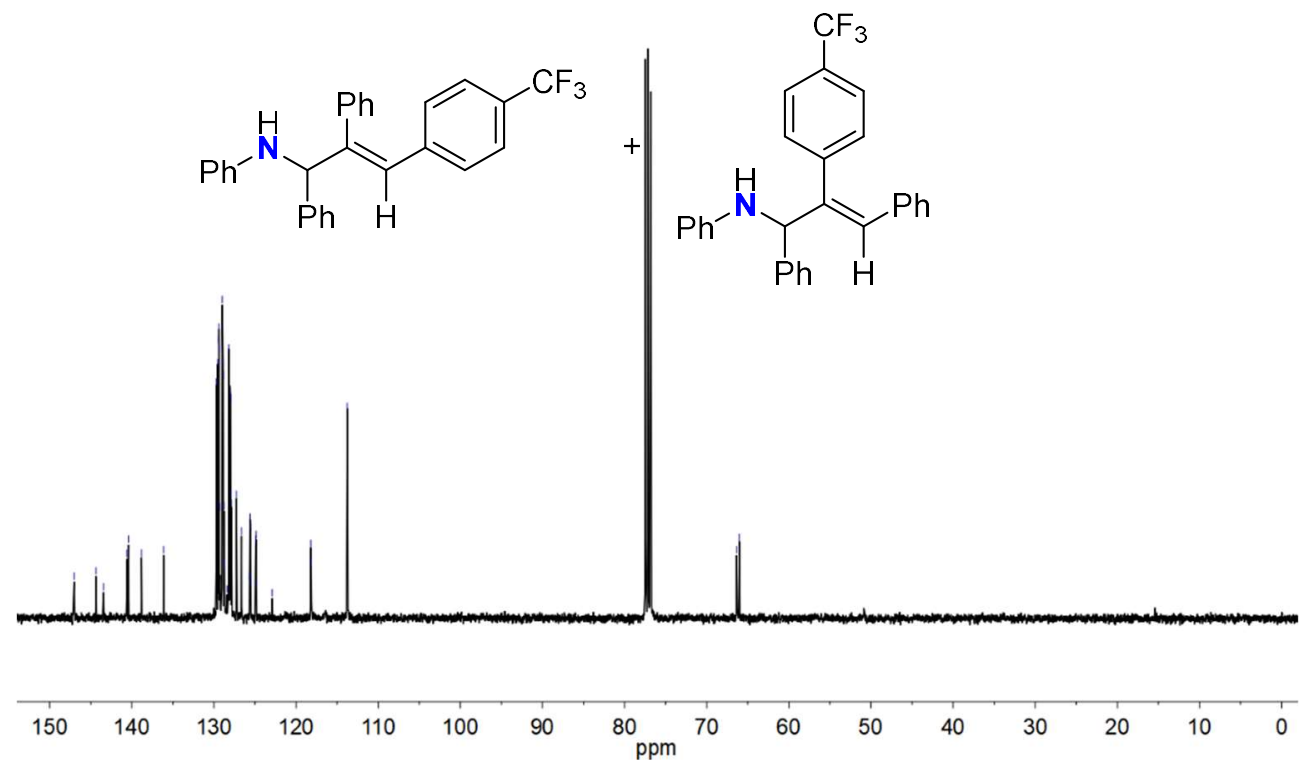
Figure S17. ${ }^{19} \mathrm{~F}\{1 \mathrm{H}\}$ NMR spectrum of $9 \mathbf{a}$ and $\mathbf{9 b}\left(\mathrm{CDCl}_{3}, 282 \mathrm{MHz}, 298 \mathrm{~K}\right)$.

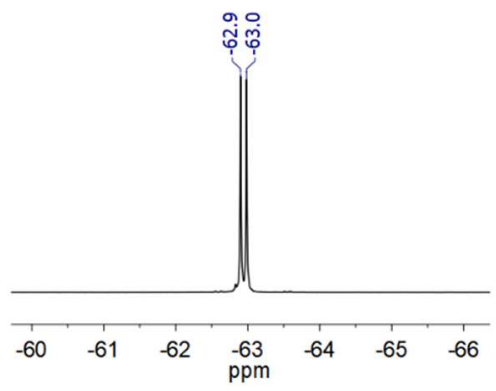<smiles>FC(F)(F)c1ccc(/C=C(\c2ccccc2)C(C=C(c2ccccc2)c2ccccc2)c2ccccc2)cc1</smiles>

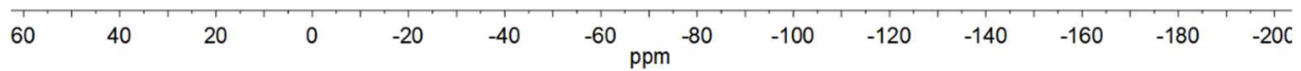

Figure S18. ${ }^{1} \mathrm{H}$ NMR spectrum of $\mathbf{1 0 a}$ and $\mathbf{1 0 b}\left(\mathrm{CDCl}_{3}, 400 \mathrm{MHz}, 298 \mathrm{~K}\right)$.
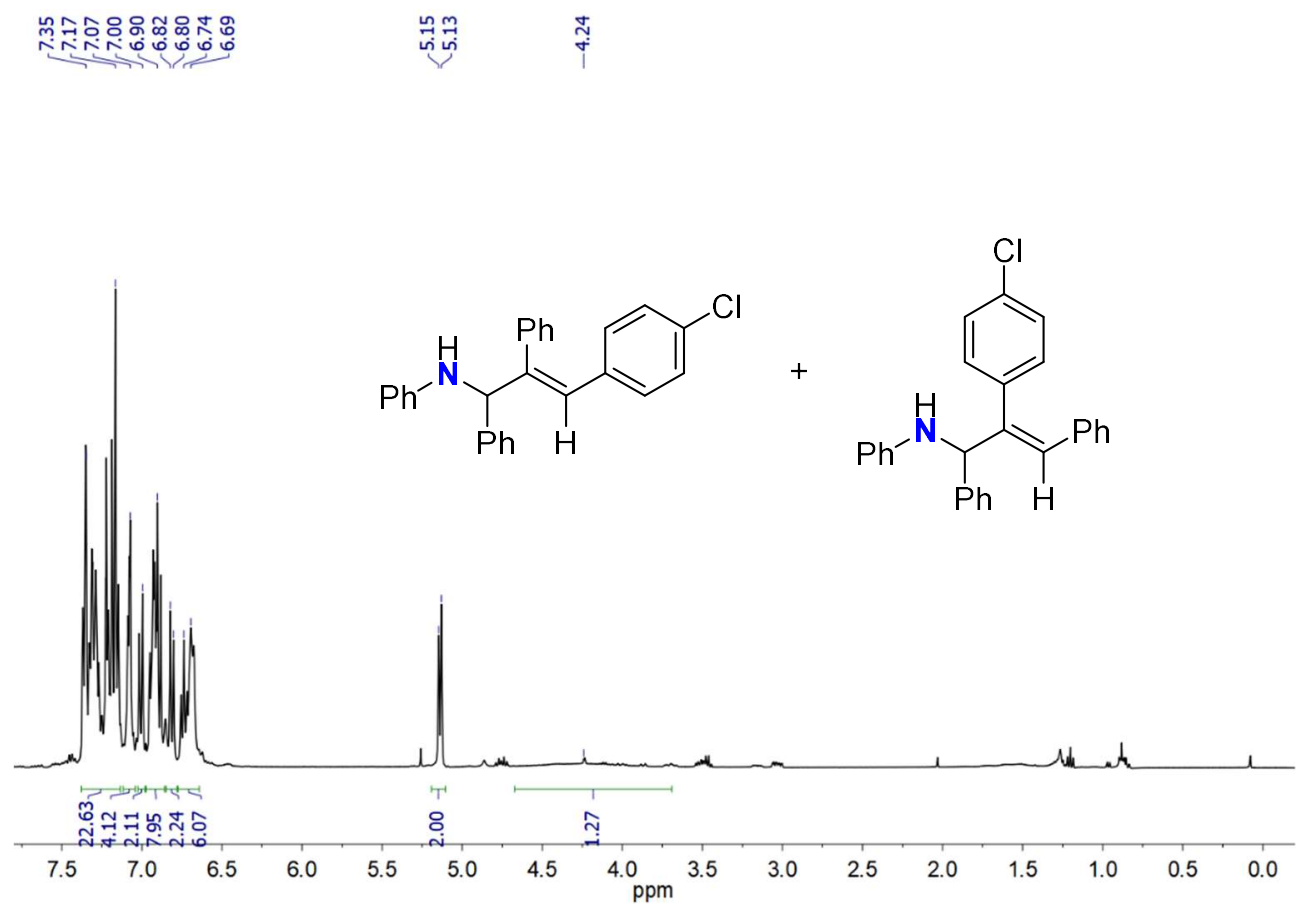
Figure S19. ${ }^{13} \mathrm{C}\left\{{ }^{1} \mathrm{H}\right\}$ NMR spectrum of $\mathbf{1 0 a}$ and $\mathbf{1 0 b}\left(\mathrm{CDCl}_{3}, 100 \mathrm{MHz}, 298 \mathrm{~K}\right)$.

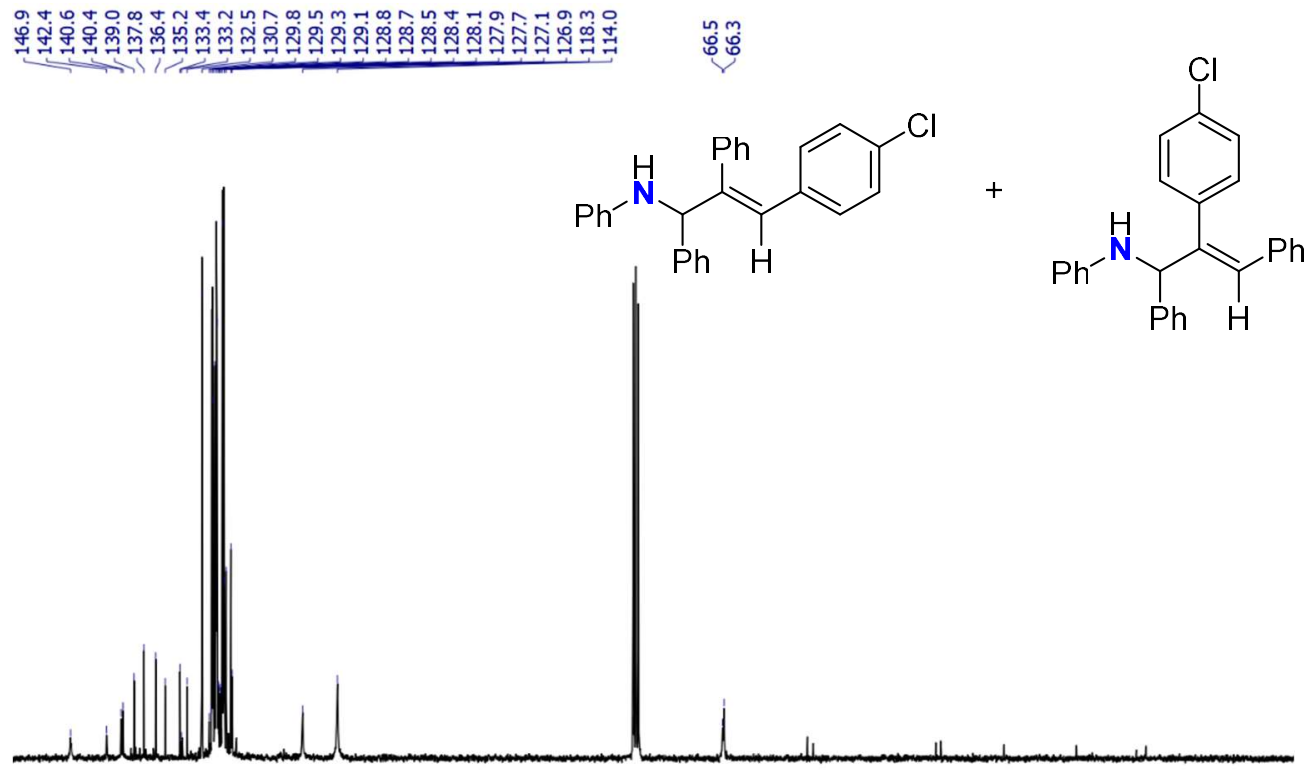

150

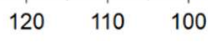

80 ppm 70

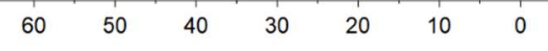

Figure S20. ${ }^{1} \mathrm{H}$ NMR spectrum of $\mathbf{1 1}\left(\mathrm{CDCl}_{3}, 400 \mathrm{MHz}, 298 \mathrm{~K}\right)$.

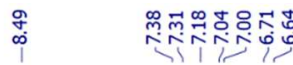

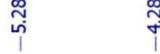

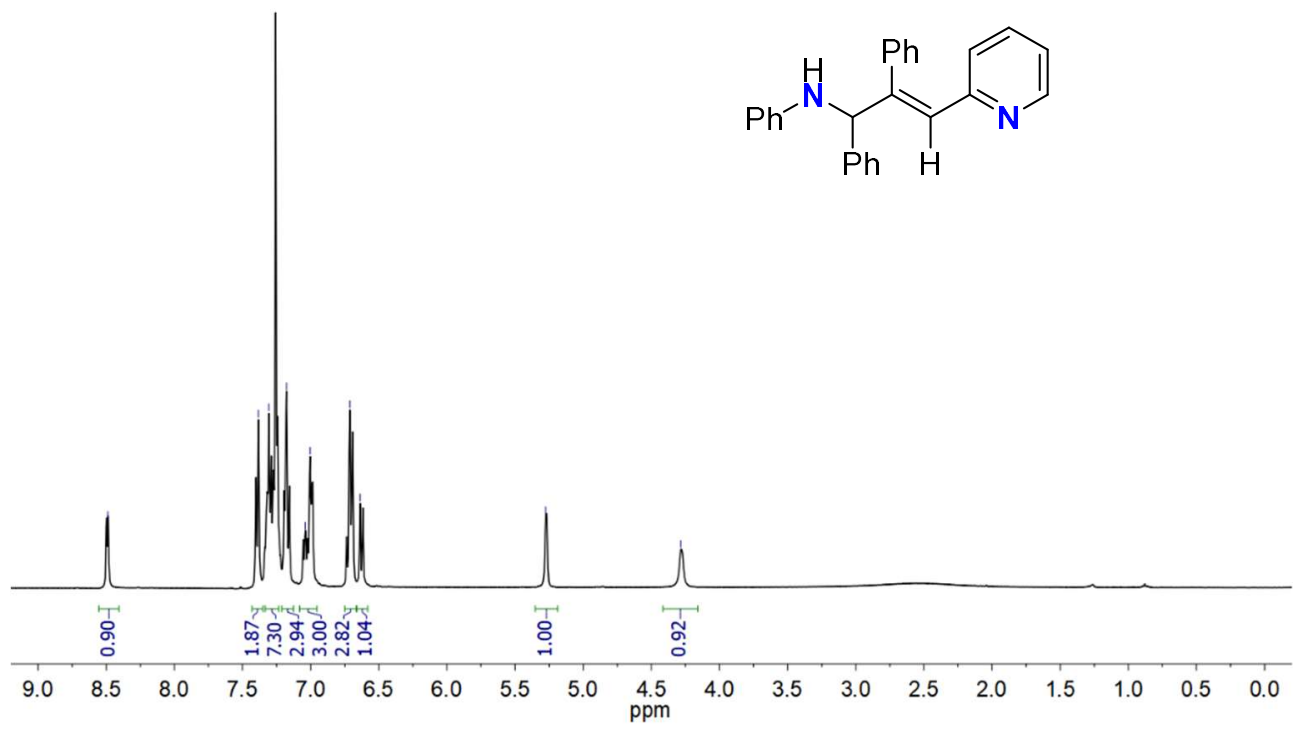


Figure S21. ${ }^{13} \mathrm{C}\left\{{ }^{1} \mathrm{H}\right\}$ NMR spectrum of $11\left(\mathrm{CDCl}_{3}, 100 \mathrm{MHz}, 298 \mathrm{~K}\right)$.

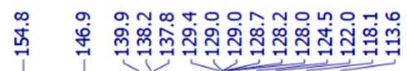

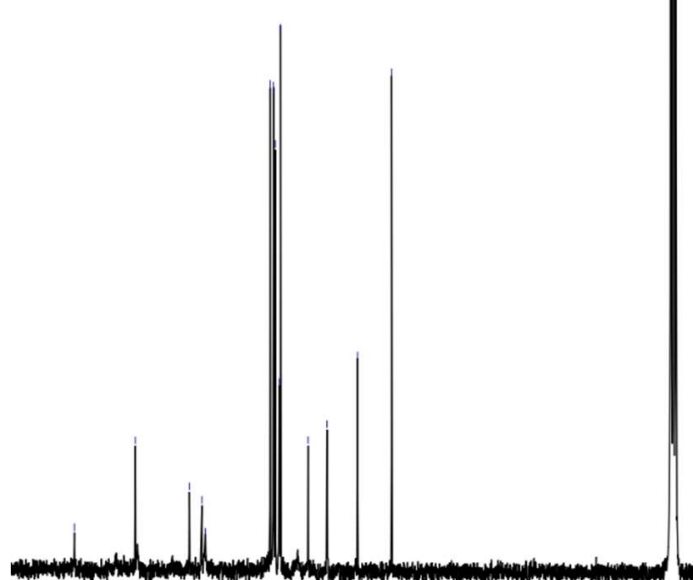

$\stackrel{\circ}{0}$<smiles>C(=C(\c1ccccc1)C(Nc1ccccc1)c1ccccc1)\c1ccccn1</smiles>

Figure S22. ${ }^{1} \mathrm{H}$ NMR spectrum of $\mathbf{1 2 a}$ and $\mathbf{1 2 b}\left(\mathrm{CDCl}_{3}, 400 \mathrm{MHz}, 298 \mathrm{~K}\right) .{ }^{11}$

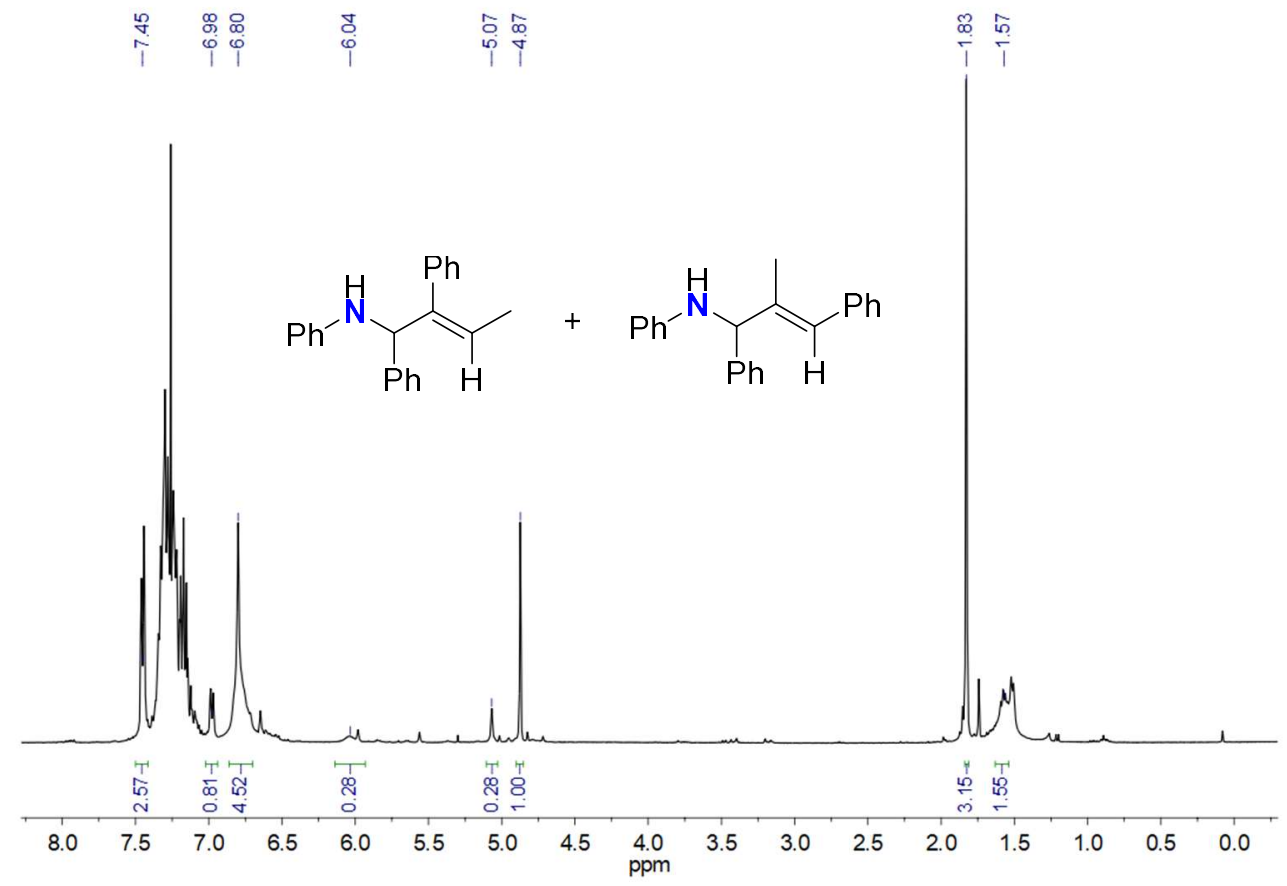


Figure S23. ${ }^{1} \mathrm{H}$ NMR spectrum of $\mathbf{1 3 a}$ and $\mathbf{1 3 b}\left(\mathrm{CDCl}_{3}, 400 \mathrm{MHz}, 298 \mathrm{~K}\right)$.

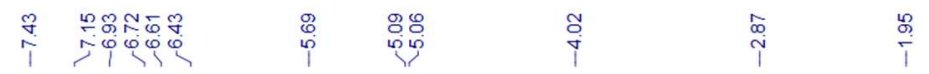

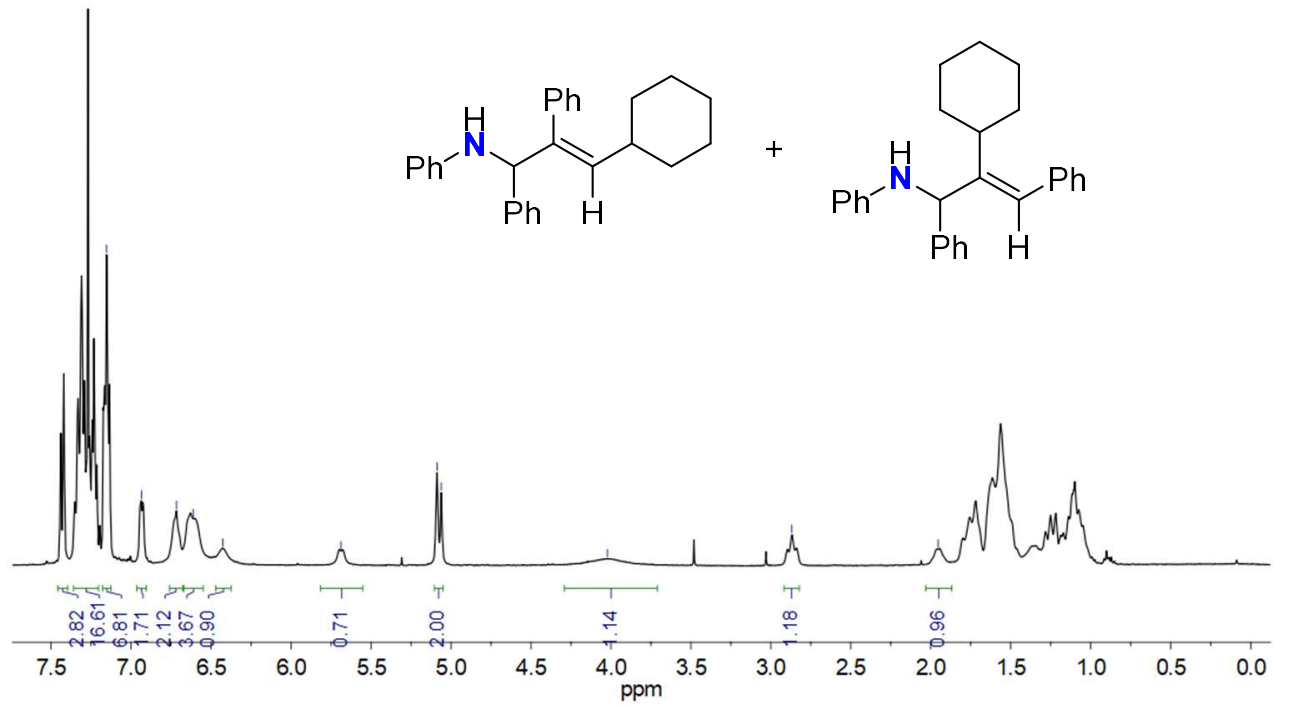

Figure S24. ${ }^{13} \mathrm{C}\left\{{ }^{1} \mathrm{H}\right\}$ NMR spectrum of $\mathbf{1 3 a}$ and $\mathbf{1 3 b}\left(\mathrm{CDCl}_{3}, 100 \mathrm{MHz}, 298 \mathrm{~K}\right)$.
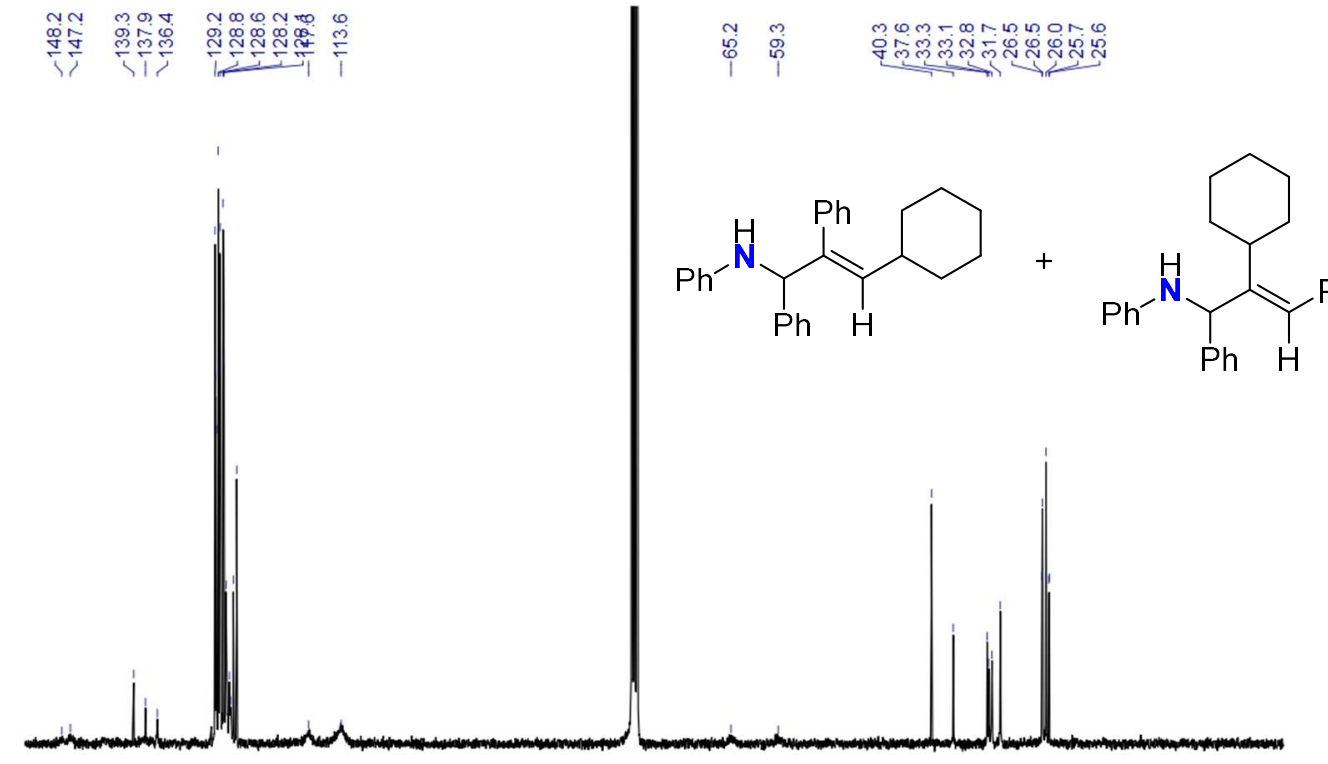

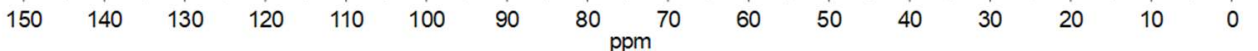


Figure S25. ${ }^{1} \mathrm{H}$ NMR spectrum of $14\left(\mathrm{CDCl}_{3}, 400 \mathrm{MHz}, 298 \mathrm{~K}\right)$.

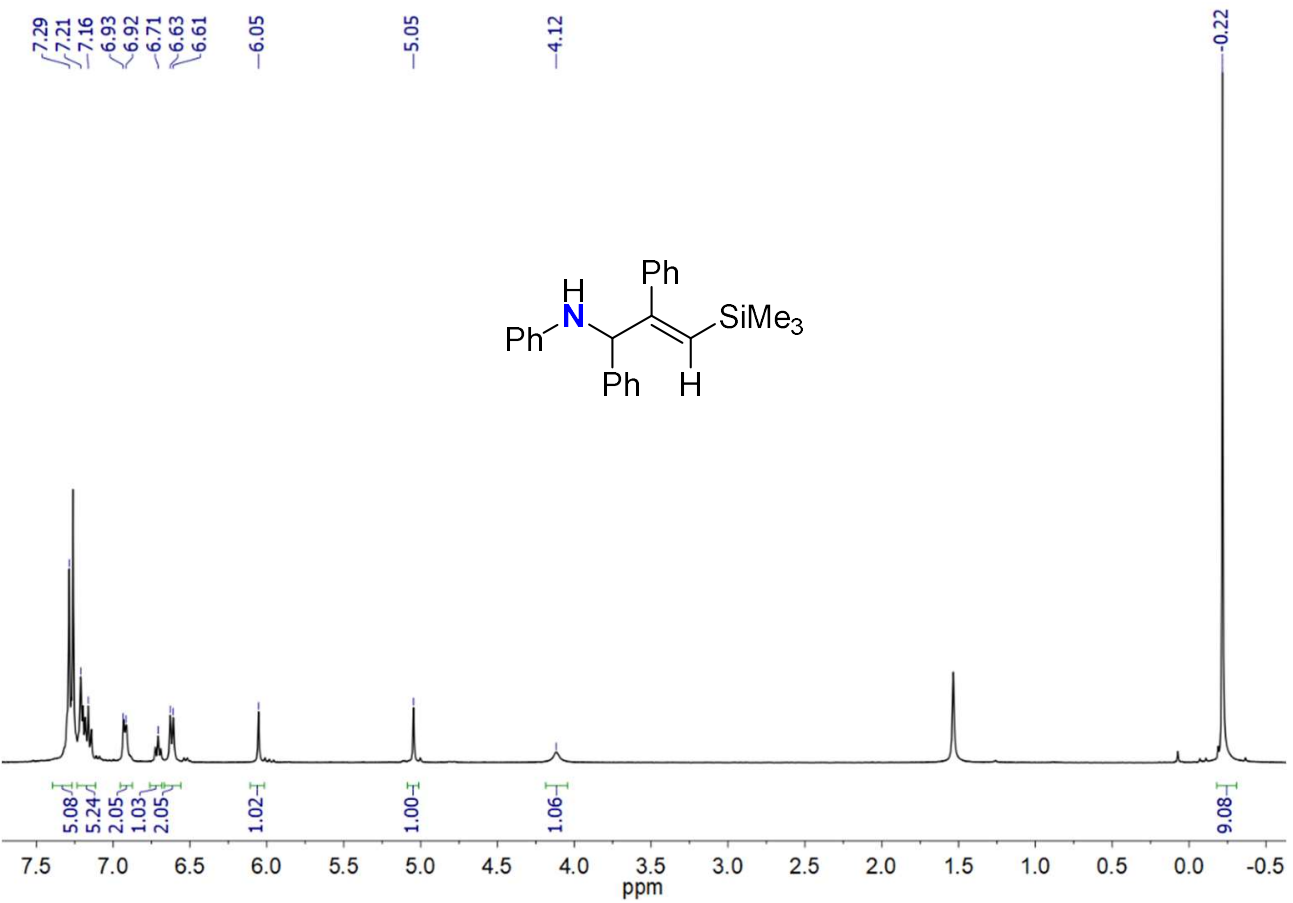

Figure S26. ${ }^{13} \mathrm{C}\left\{{ }^{1} \mathrm{H}\right\}$ NMR spectrum of $14\left(\mathrm{CDCl}_{3}, 100 \mathrm{MHz}, 298 \mathrm{~K}\right)$.

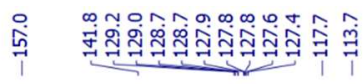

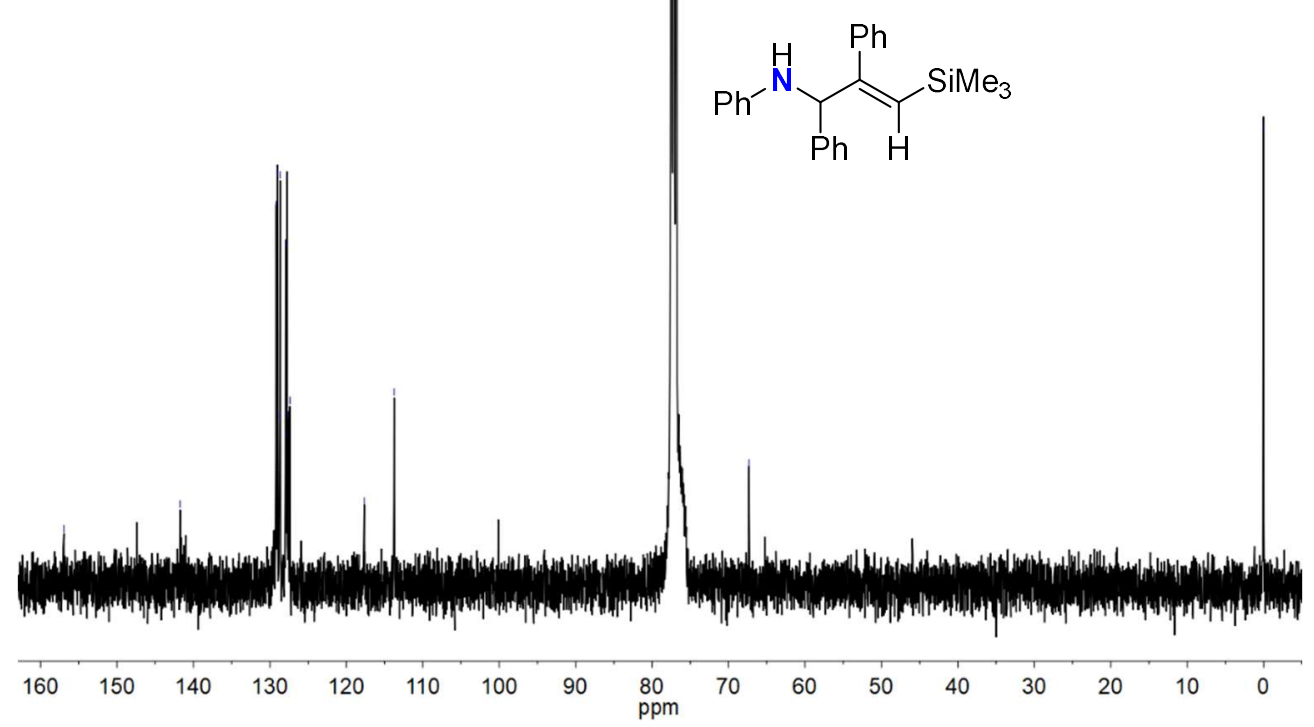


Figure S27. ${ }^{1} \mathrm{H}$ NMR spectrum of $15\left(\mathrm{CDCl}_{3}, 300 \mathrm{MHz}, 298 \mathrm{~K}\right)$.

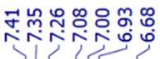

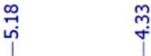<smiles>Cc1ccc(NC(/C(=C/c2ccccc2)c2ccccc2)c2ccccc2)cc1</smiles>

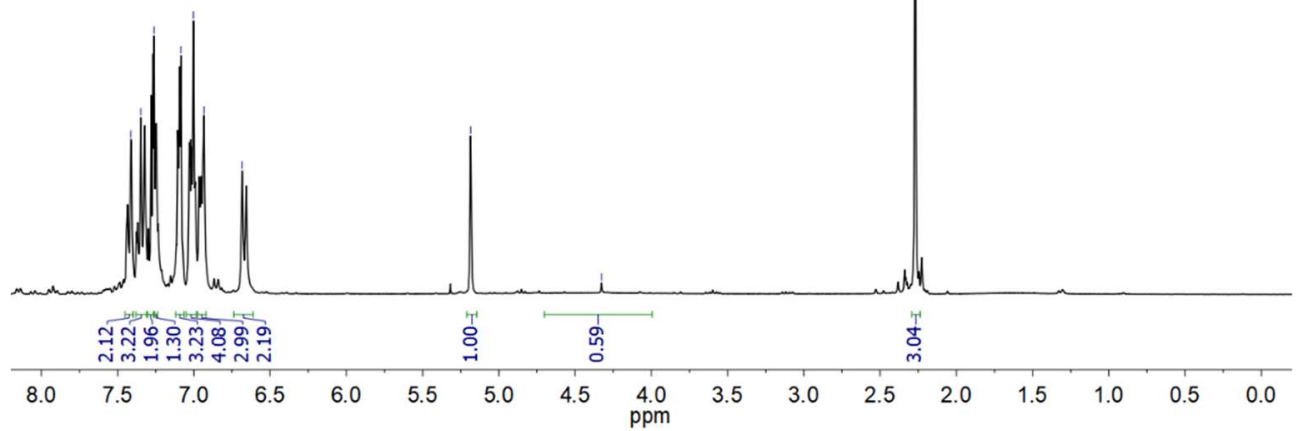

Figure S28. ${ }^{13} \mathrm{C}\left\{{ }^{1} \mathrm{H}\right\}$ NMR spectrum of $\mathbf{1 5}\left(\mathrm{CDCl}_{3}, 75 \mathrm{MHz}, 298 \mathrm{~K}\right)$.
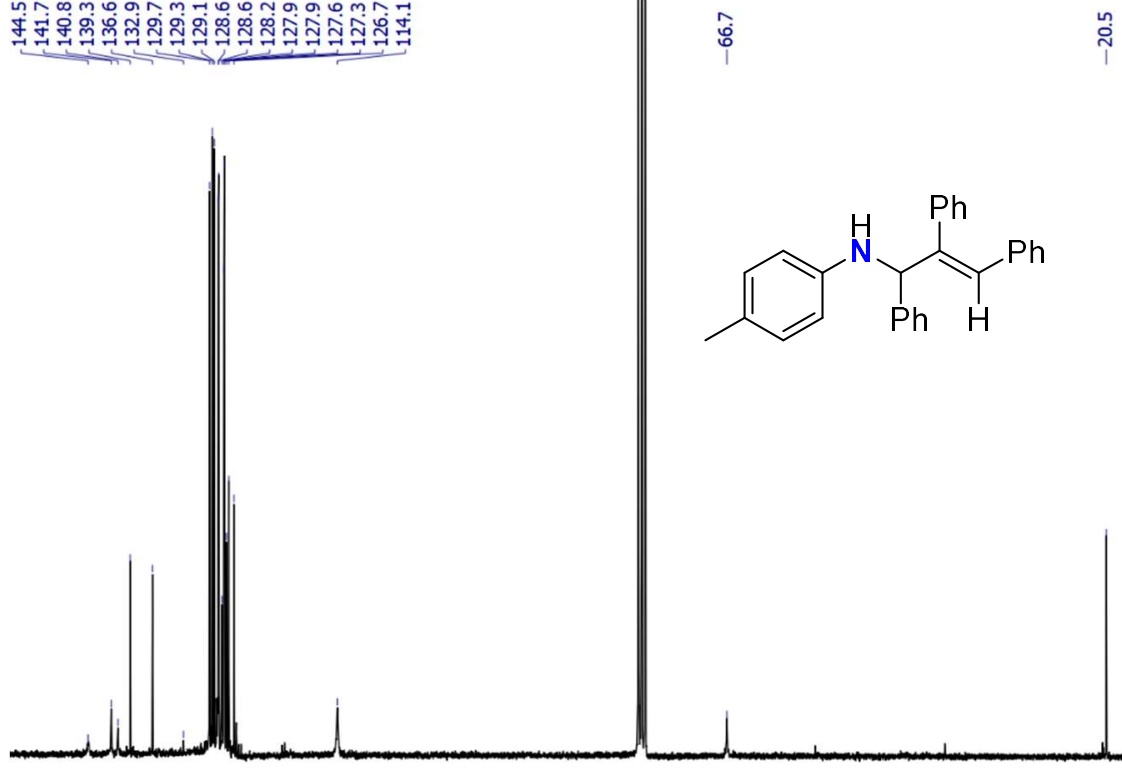<smiles>Cc1ccc(NC(/C(=C/c2ccccc2)c2ccccc2)c2ccccc2)cc1</smiles>

150
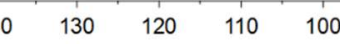

90 $80 \mathrm{ppm} 70$ 
Figure S29. ${ }^{1} \mathrm{H}$ NMR spectrum of $\mathbf{1 6}\left(\mathrm{CDCl}_{3}, 400 \mathrm{MHz}, 298 \mathrm{~K}\right)$.

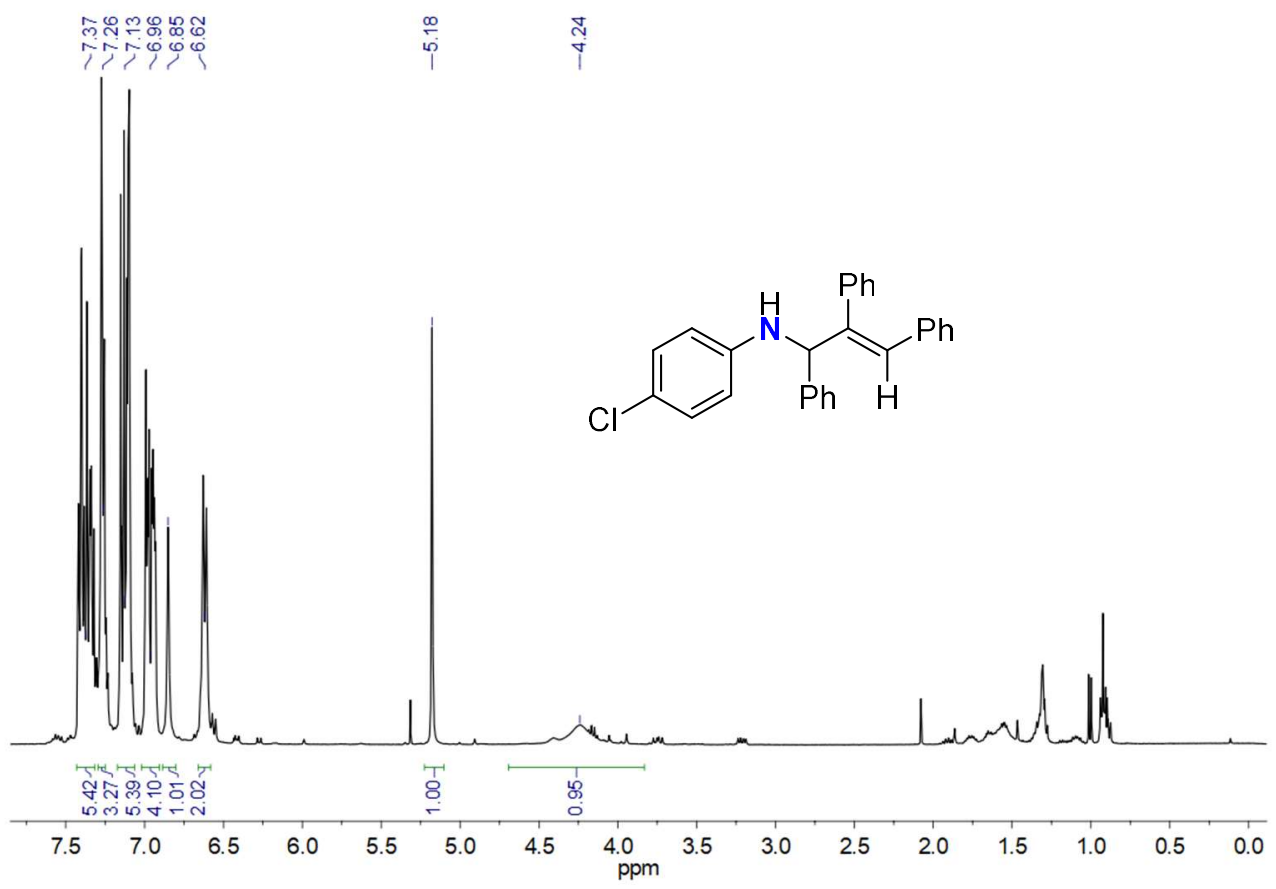

Figure S30. ${ }^{13} \mathrm{C}\left\{{ }^{1} \mathrm{H}\right\}$ NMR spectrum of $16\left(\mathrm{CDCl}_{3}, 100 \mathrm{MHz}, 298 \mathrm{~K}\right)$.

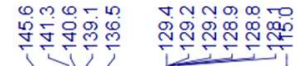

@ొ

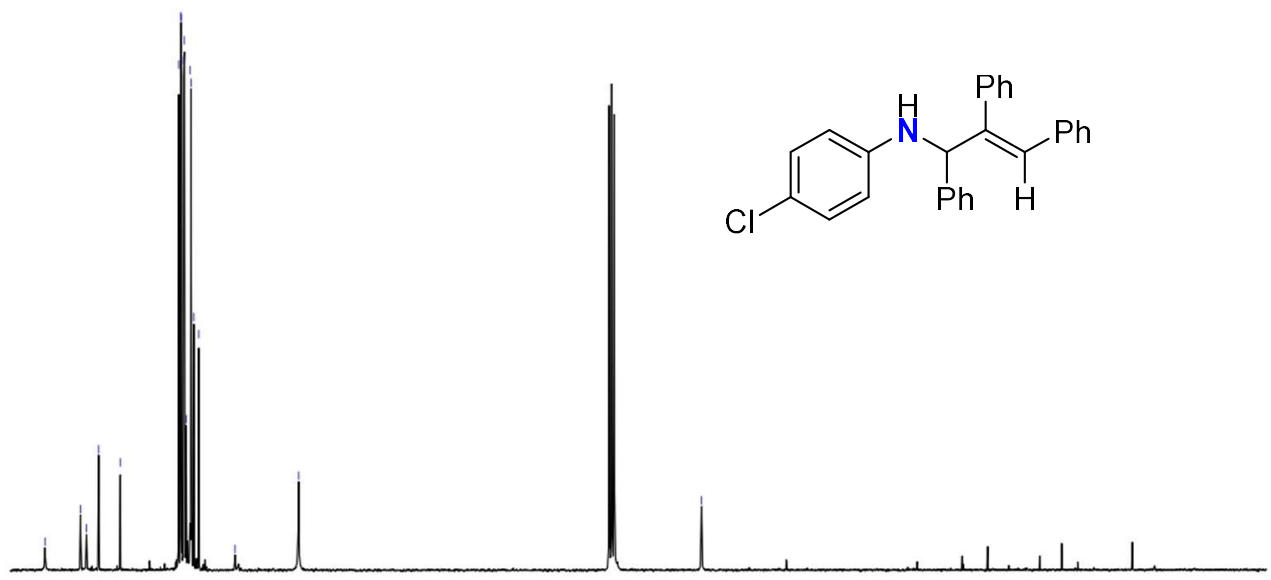

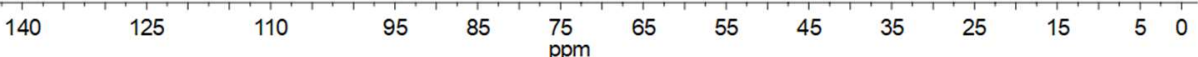


Figure S31. ${ }^{1} \mathrm{H}$ NMR spectrum of $17\left(\mathrm{CDCl}_{3}, 400 \mathrm{MHz}, 298 \mathrm{~K}\right)$.
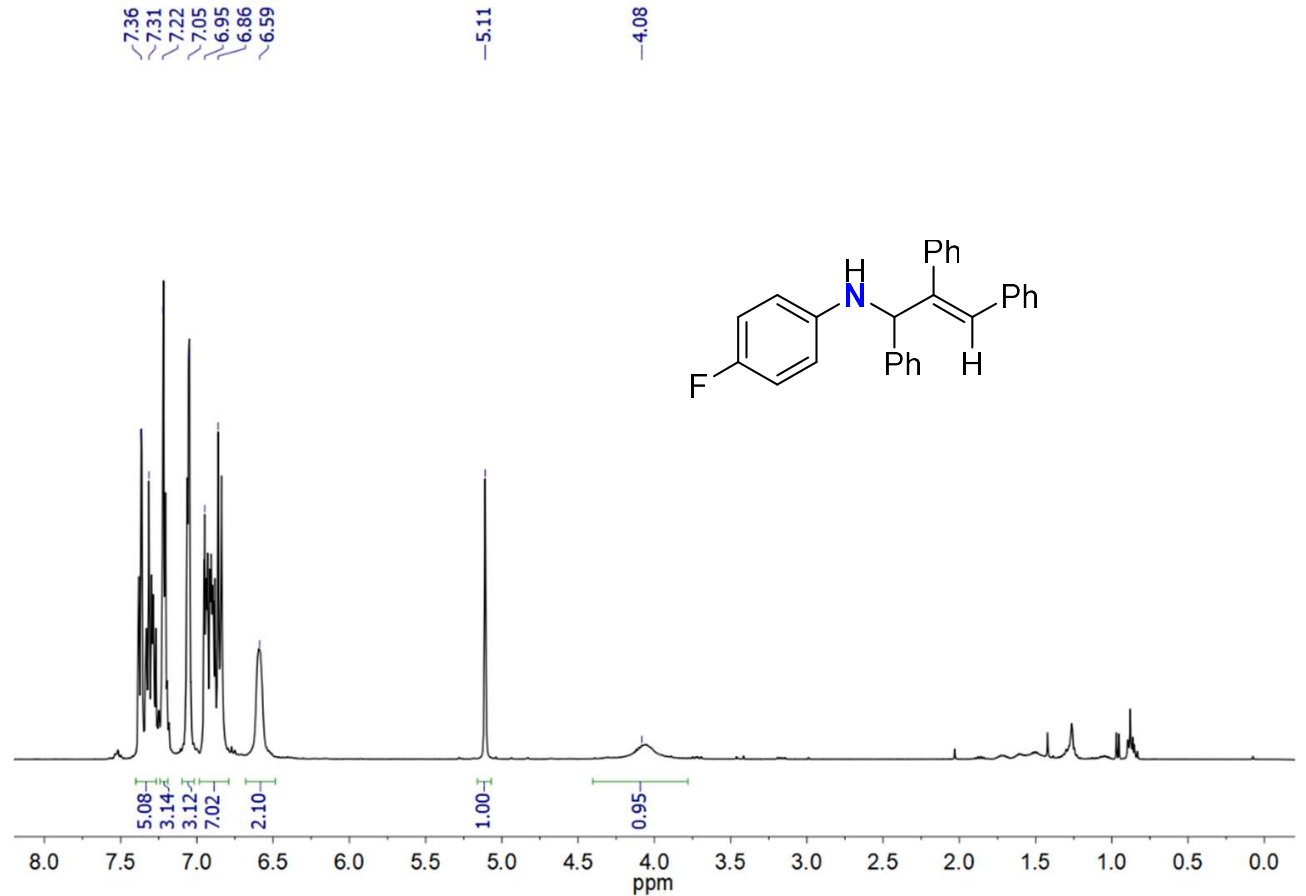

Figure S32. ${ }^{13} \mathrm{C}\left\{{ }^{1} \mathrm{H}\right\}$ NMR spectrum of $17\left(\mathrm{CDCl}_{3}, 100 \mathrm{MHz}, 298 \mathrm{~K}\right)$.

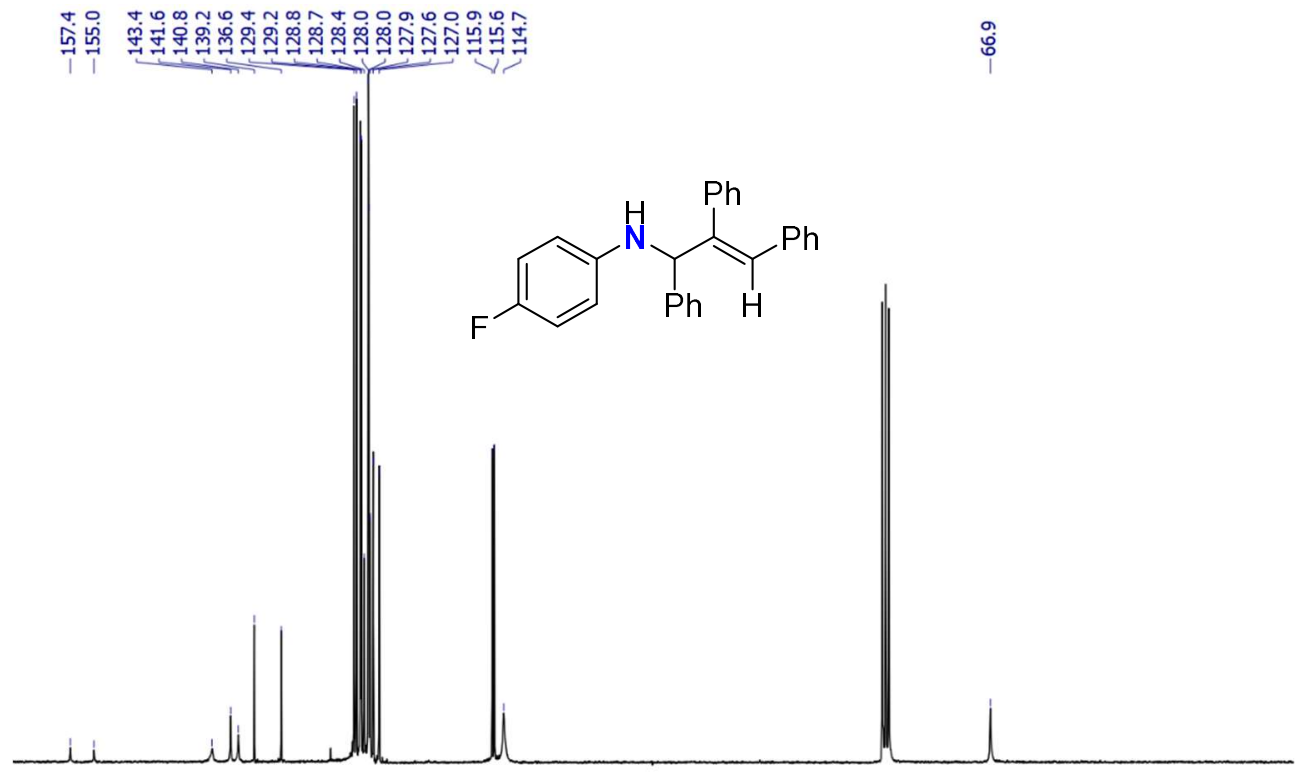

$\begin{array}{llllllllllllllllllllllllllllllllll}160 & 155 & 150 & 145 & 140 & 135 & 130 & 125 & 120 & 115 & 110 & 105 & 100 & 95 & 90 & 85 & 80 & 75 & 70 & 65 & 60 & 55 & 50 & 45 & 40\end{array}$ 
Figure S33. ${ }^{19} \mathrm{~F}\left\{{ }^{1} \mathrm{H}\right\}$ NMR spectrum of $17\left(\mathrm{CDCl}_{3}, 282 \mathrm{MHz}, 298 \mathrm{~K}\right)$.

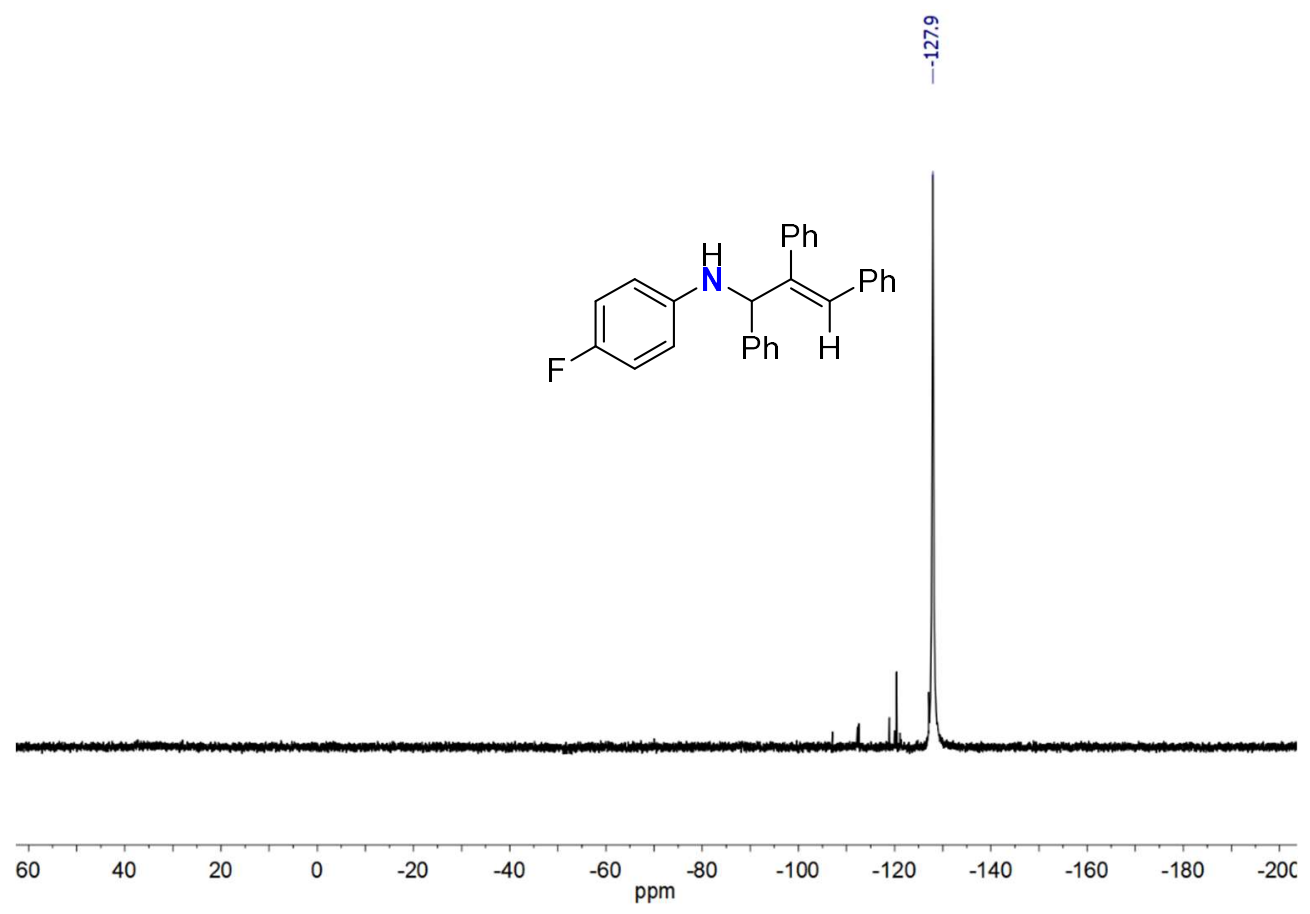

Figure S34. ${ }^{1} \mathrm{H}$ NMR spectrum of $18\left(\mathrm{CDCl}_{3}, 400 \mathrm{MHz}, 298 \mathrm{~K}\right)$.

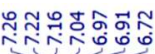

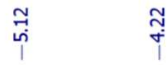

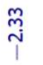

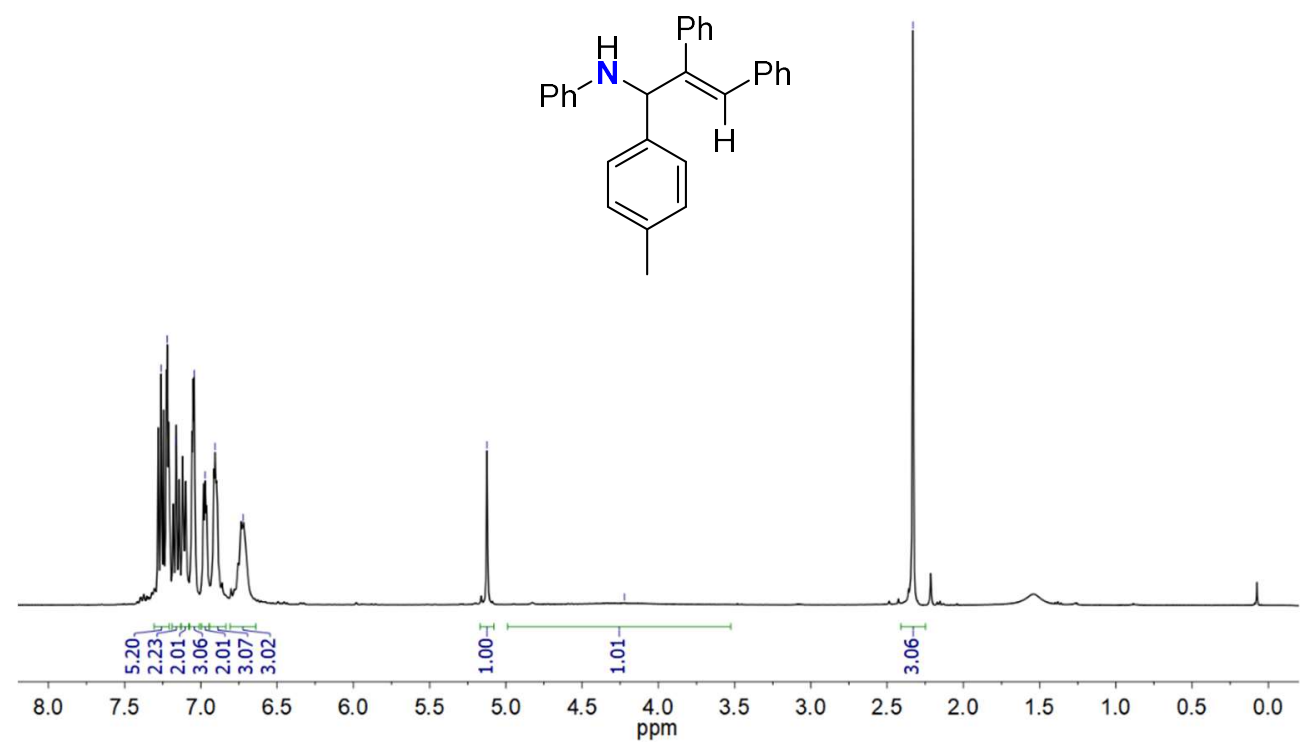


Figure S35. ${ }^{13} \mathrm{C}\left\{{ }^{1} \mathrm{H}\right\}$ NMR spectrum of $18\left(\mathrm{CDCl}_{3}, 100 \mathrm{MHz}, 298 \mathrm{~K}\right)$.

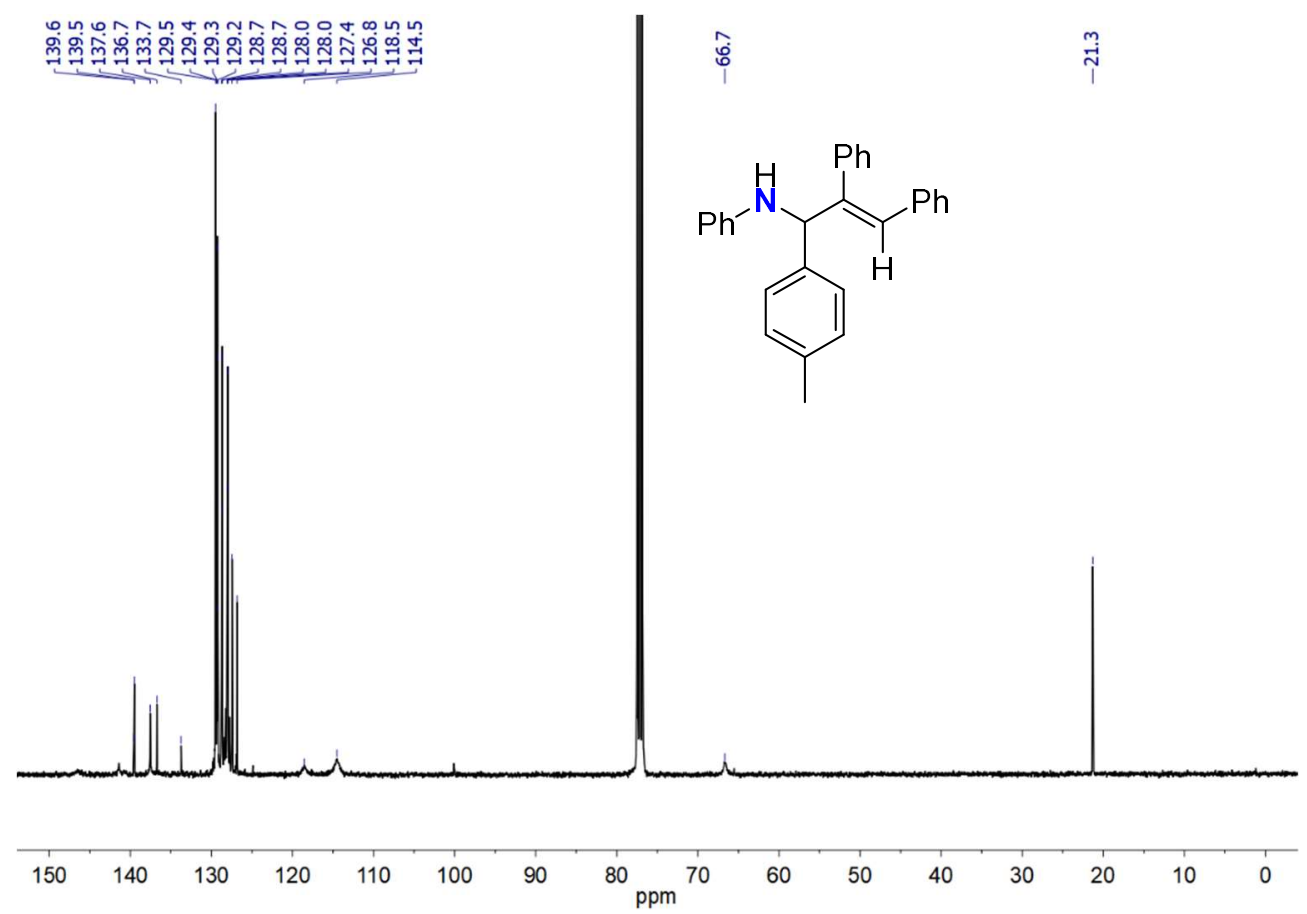

Figure S36. ${ }^{1} \mathrm{H}$ NMR spectrum of $19\left(\mathrm{CDCl}_{3}, 400 \mathrm{MHz}, 298 \mathrm{~K}\right)$.

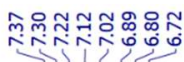

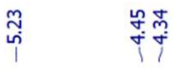

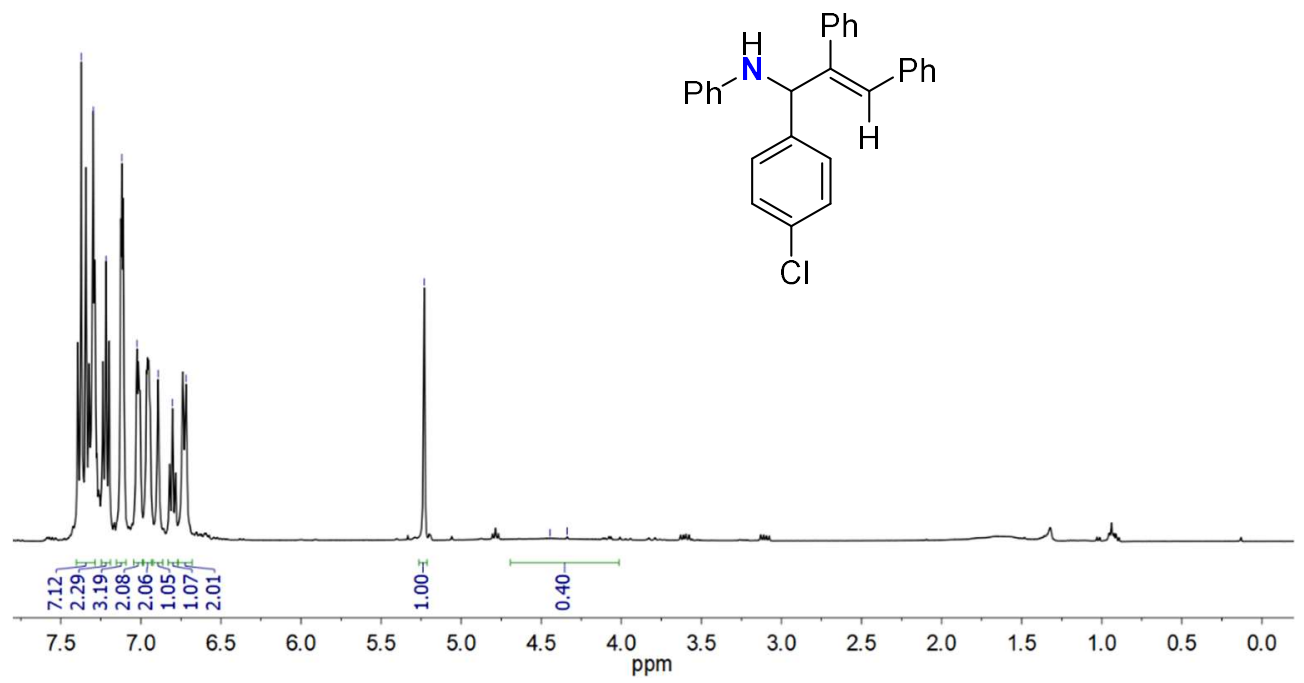


Figure S37. ${ }^{13} \mathrm{C}\left\{{ }^{1} \mathrm{H}\right\}$ NMR spectrum of $19\left(\mathrm{CDCl}_{3}, 100 \mathrm{MHz}, 298 \mathrm{~K}\right)$.

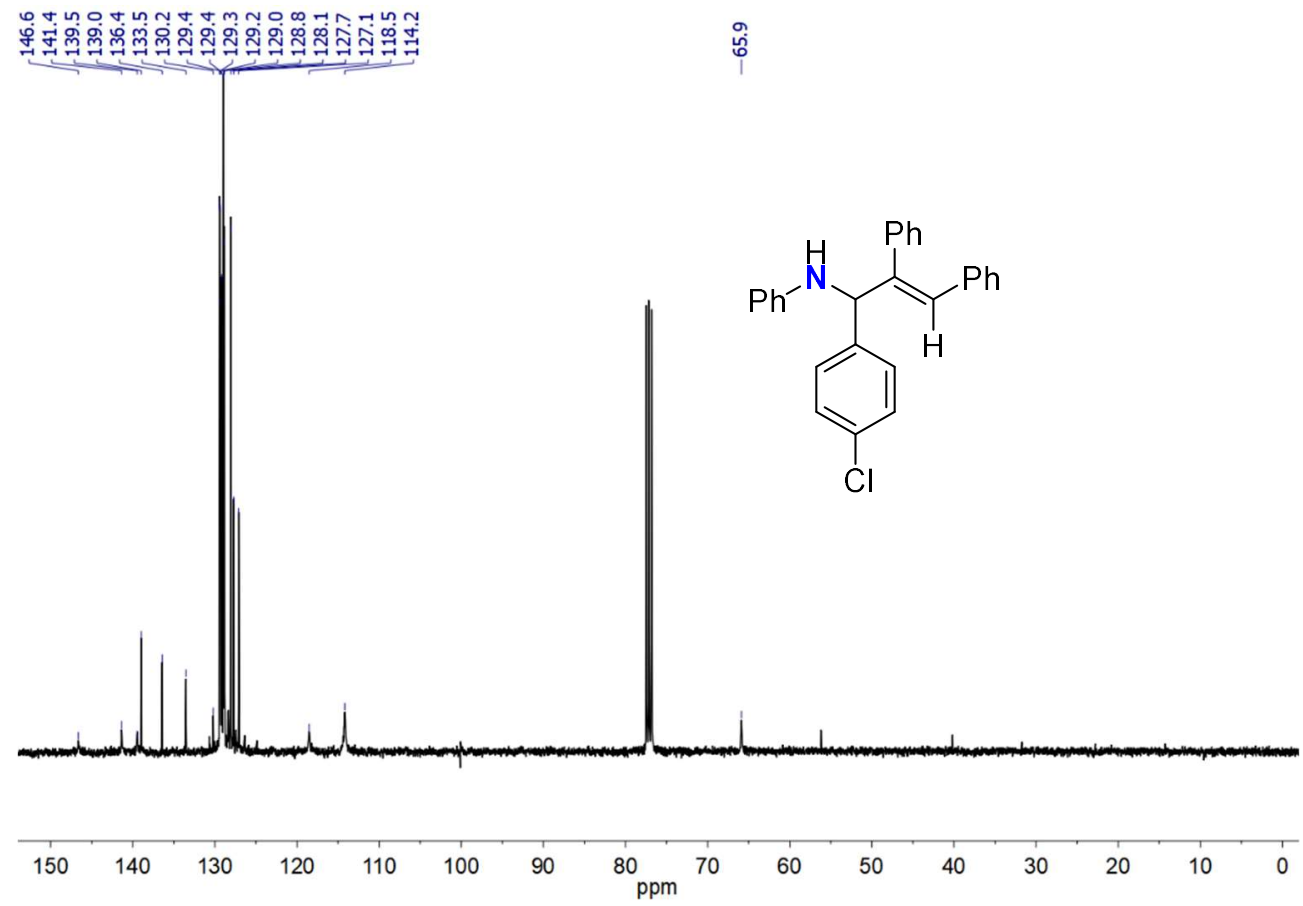

Figure S38. ${ }^{1} \mathrm{H}$ NMR spectrum of $20\left(\mathrm{CDCl}_{3}, 400 \mathrm{MHz}, 298 \mathrm{~K}\right)$.
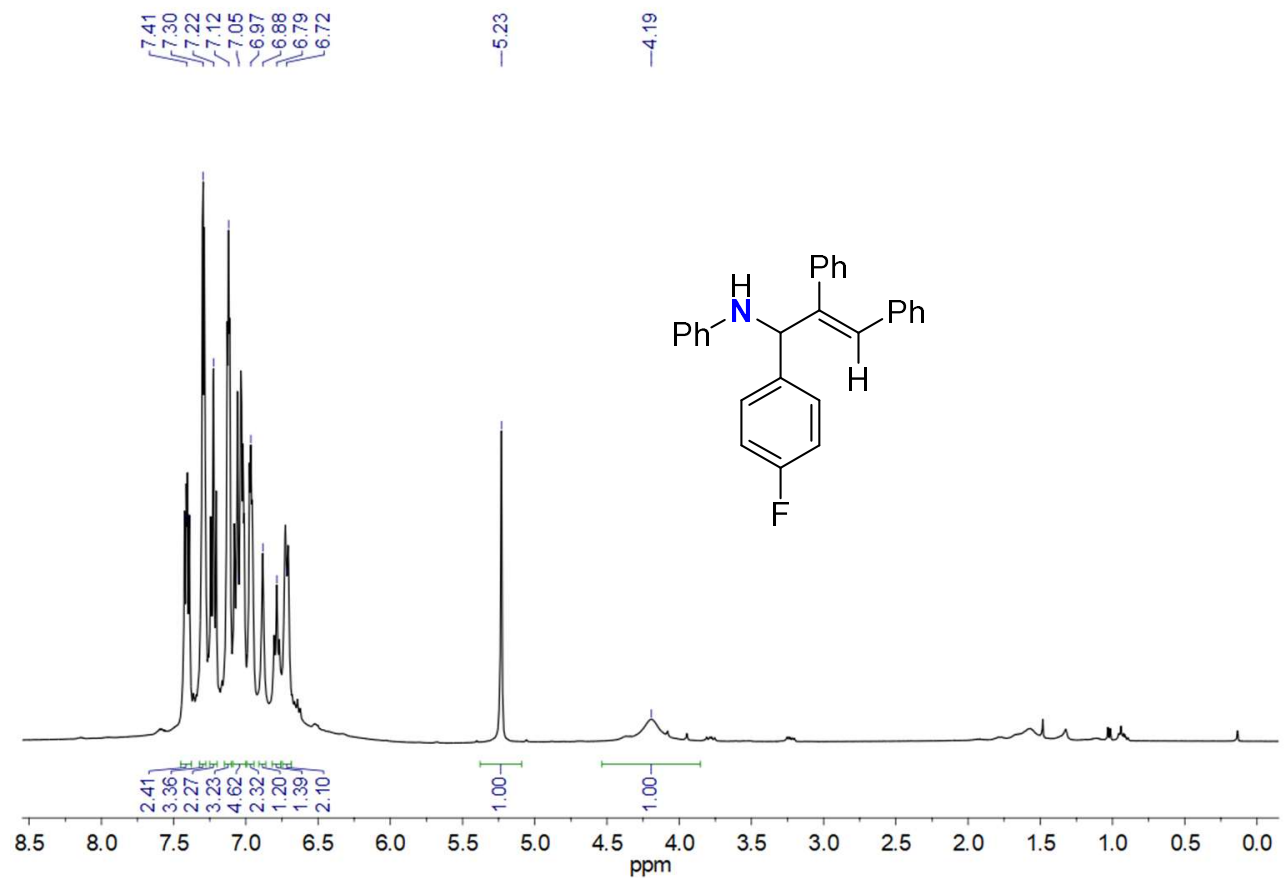
Figure S39. ${ }^{13} \mathrm{C}\left\{{ }^{1} \mathrm{H}\right\}$ NMR spectrum of $\mathbf{2 0}\left(\mathrm{CDCl}_{3}, 100 \mathrm{MHz}, 298 \mathrm{~K}\right)$.

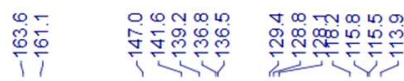

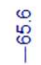
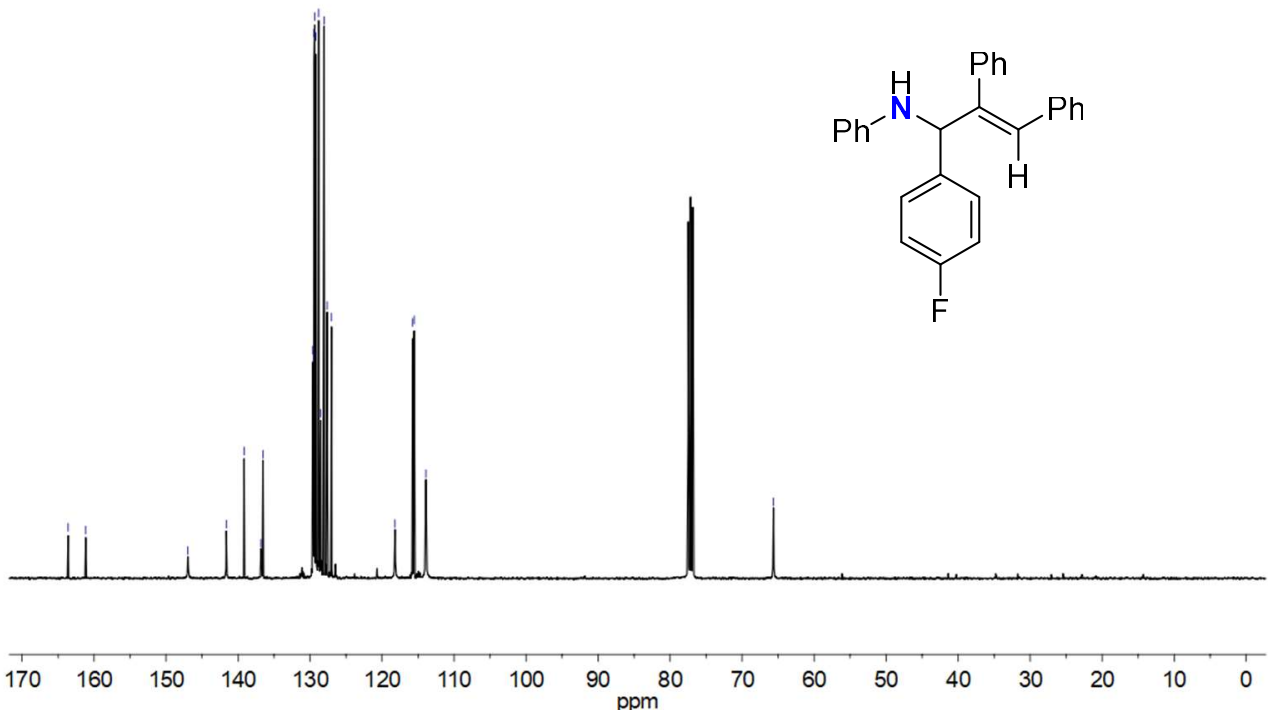

Figure S40. ${ }^{19} \mathrm{~F}\left\{{ }^{1} \mathrm{H}\right\}$ NMR spectrum of $20\left(\mathrm{CDCl}_{3}, 282 \mathrm{MHz}, 298 \mathrm{~K}\right)$.

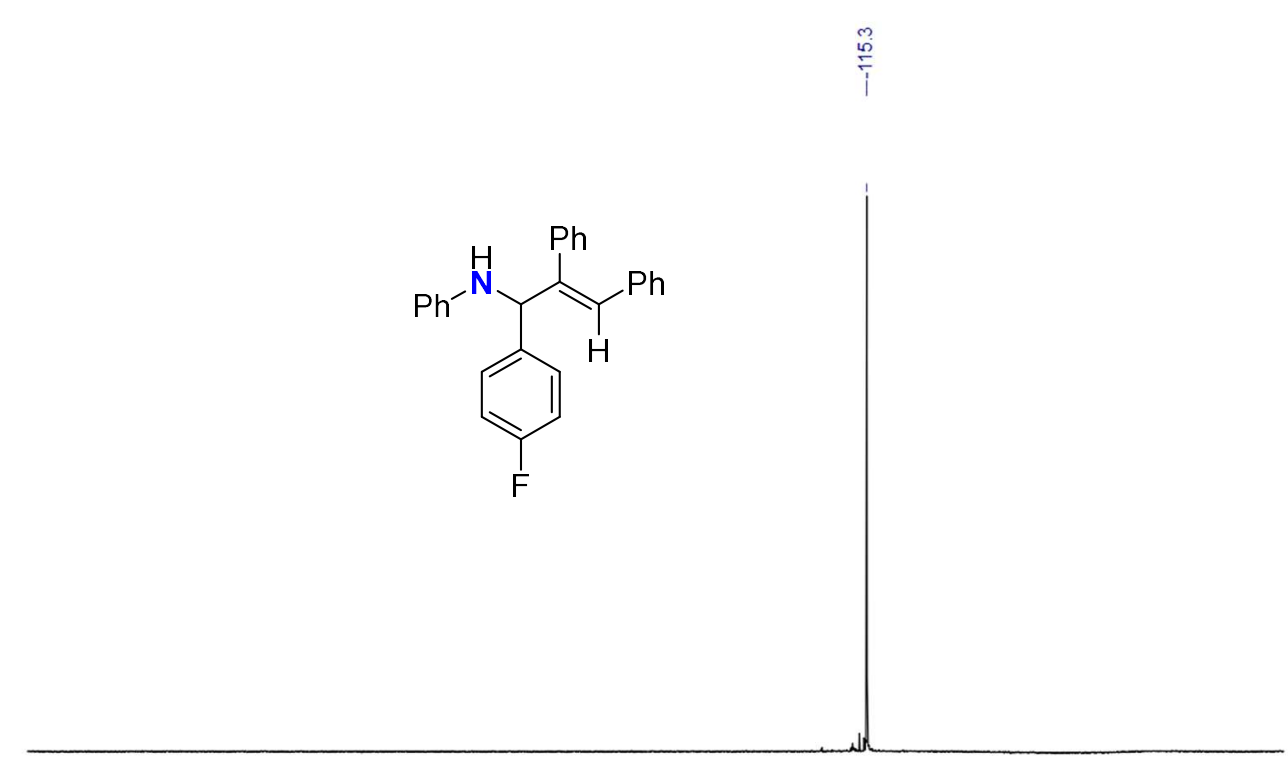

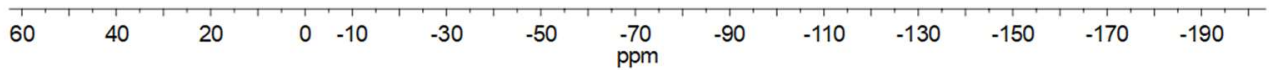


Figure S41. ${ }^{1} \mathrm{H}$ NMR of the catalytic reaction with $N$-(trimethylsilyl)benzylamine $\left(\mathrm{C}_{6} \mathrm{D}_{6}, 300\right.$ $\mathrm{MHz}, 298 \mathrm{~K})$.

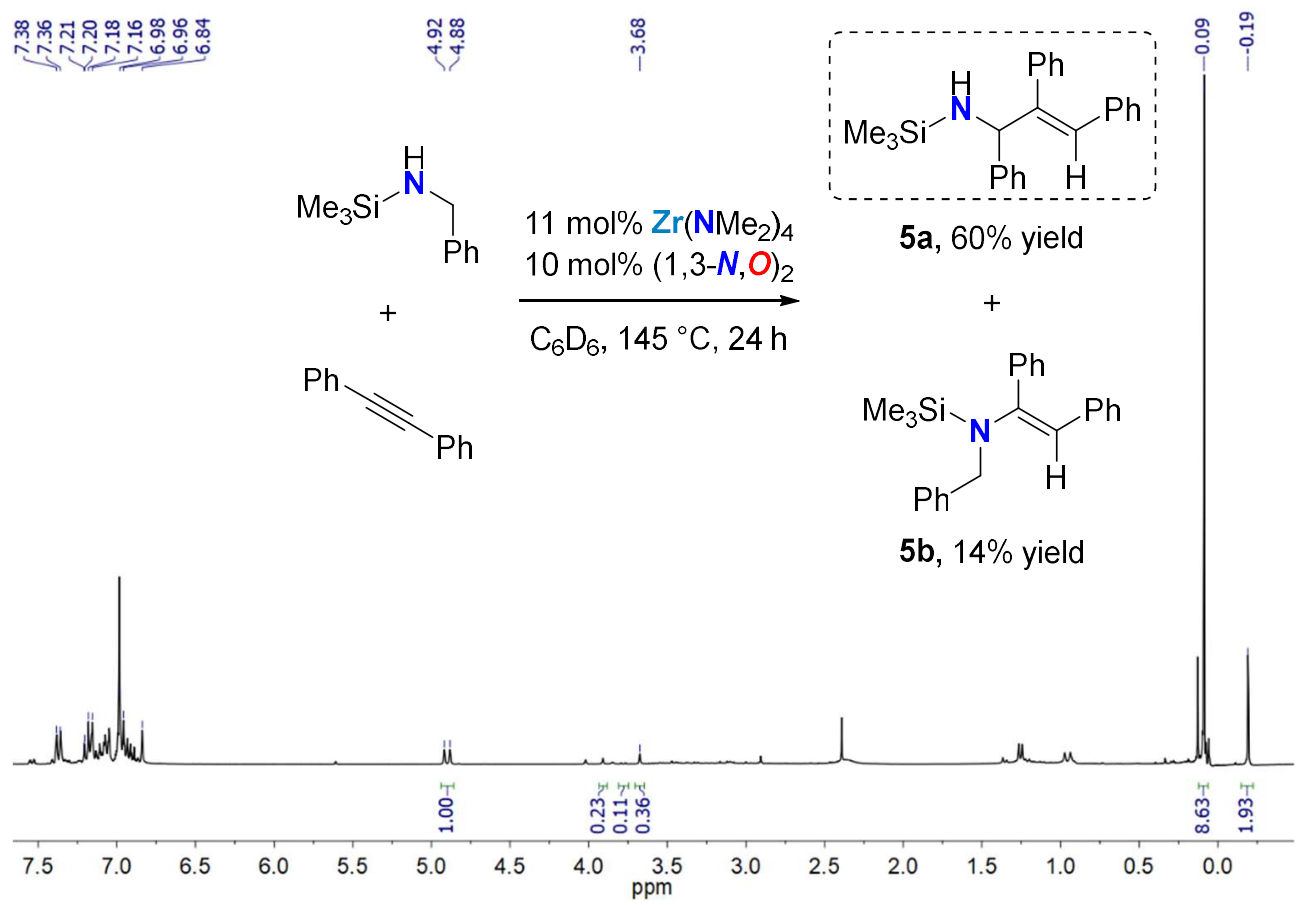

Figure S42. ${ }^{1} \mathrm{H}$ NMR of the initial catalytic reaction with $N$-benzylaniline $\left(\mathrm{C}_{6} \mathrm{D}_{6}, 300 \mathrm{MHz}, 298\right.$ $\mathrm{K})$.

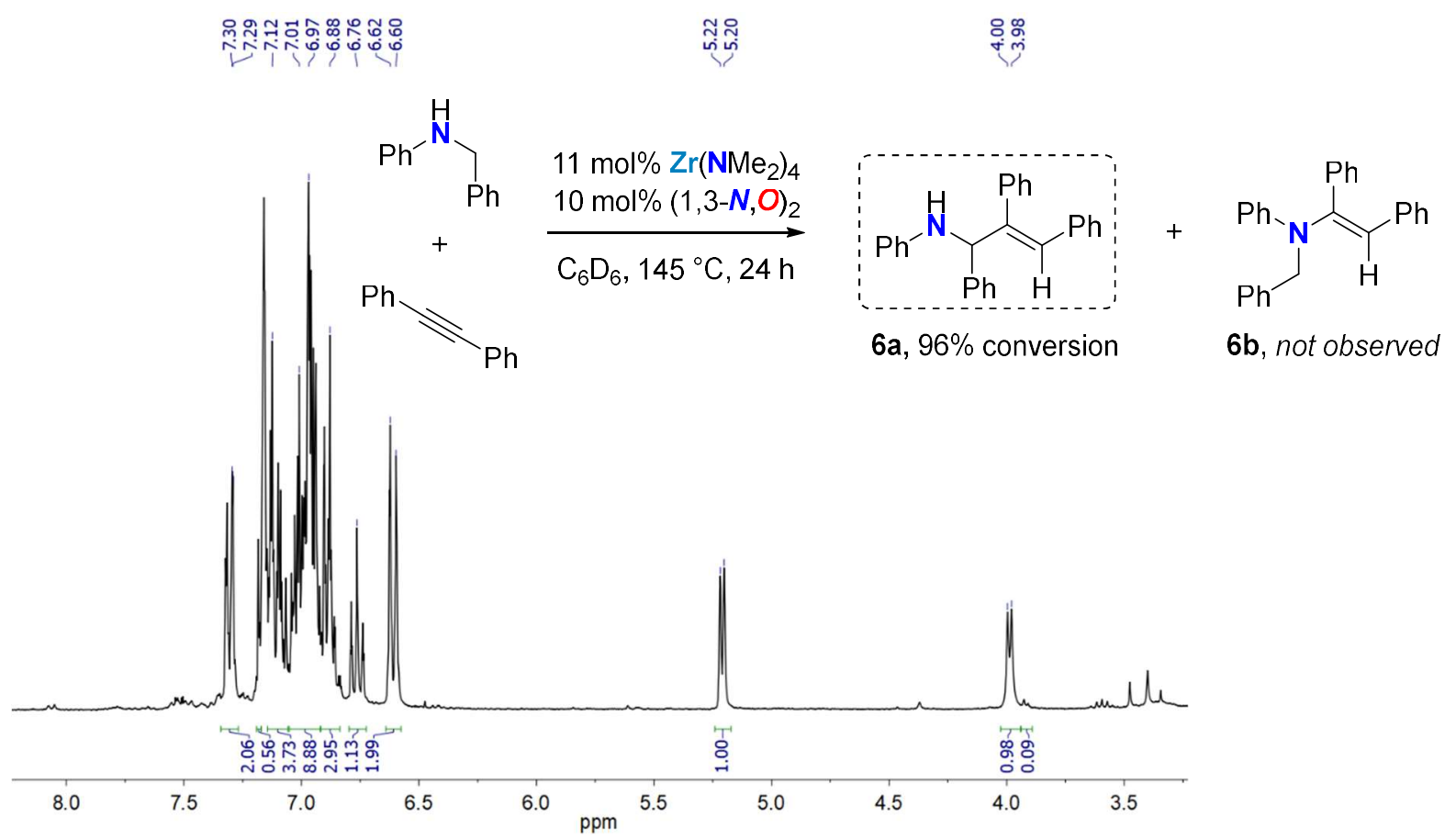


Figure S43. ${ }^{1} \mathrm{H}$ NMR of the catalytic reaction in the absence of ligand $\left(\mathrm{C}_{6} \mathrm{D}_{6}, 300 \mathrm{MHz}, 298 \mathrm{~K}\right)$.

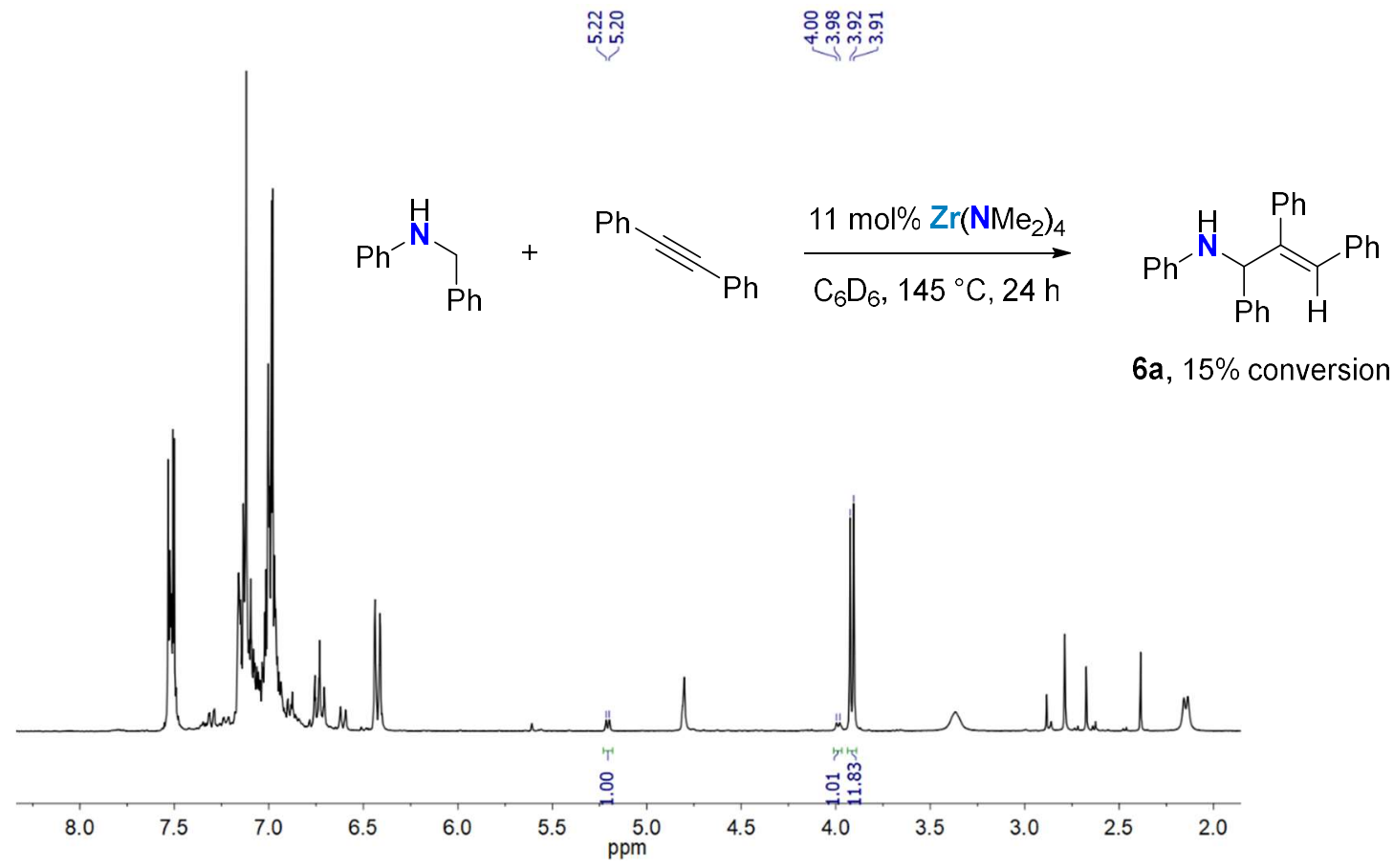

Figure S44. ${ }^{1} \mathrm{H}$ NMR of the catalytic reaction using catalyst $\mathbf{E}\left(\mathrm{C}_{6} \mathrm{D}_{6}, 300 \mathrm{MHz}, 298 \mathrm{~K}\right)$.

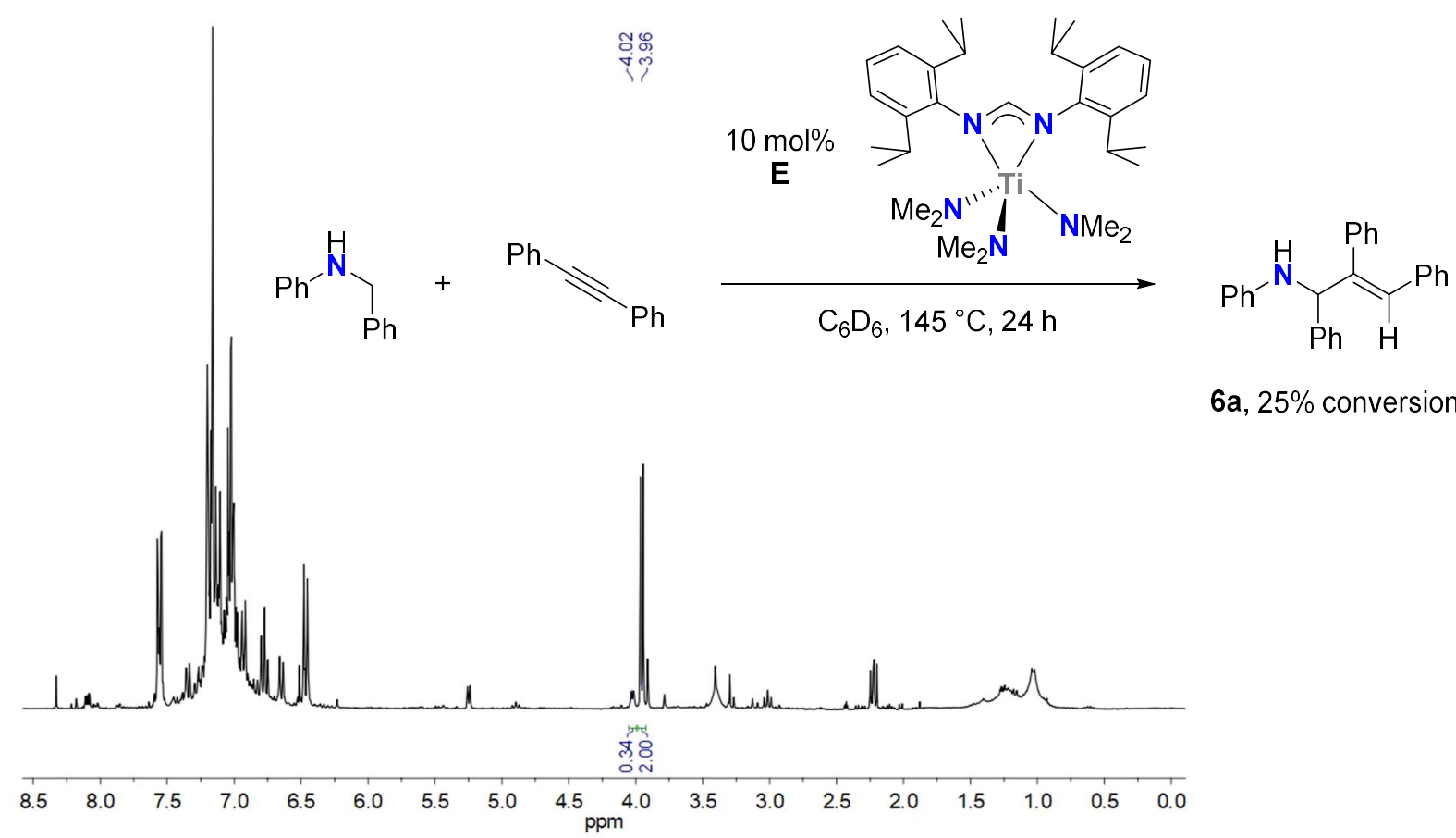




\section{Crystallographic details}

A summary of the crystallographic data for compounds $\mathbf{1 , 2 , 3}$, and $\mathbf{1 9}$ is shown in Table $\mathrm{S} 1$. The automatic data collection strategy was determined using COSMO and the cell determination and integration processes were carried out using SAINT. Using Olex $2{ }^{12}$ the structures were solved with the ShelXT $T^{13}$ structure solution program using Intrinsic Phasing and the structures were refined using the ShelXL ${ }^{14}$ refinement package using the Least Squares method. PLATON/SQUEEZE ${ }^{15}$ was used in the structural refinement of $\mathbf{3}$ to remove disordered hexanes. Complex $\mathbf{1}$ was poorlydiffracting, resulting in relatively poor resolution and two unusually shaped ellipsoids. This could not be improved to resolve the A- and B-alerts in the checkCIF for $\mathbf{1}$. Complex $\mathbf{3}$ was also poorlydiffracting, resulting in relatively poor resolution and four unusually shaped ellipsoids. Additionally, $\mathbf{3}$ crystallizes as a racemic twin in a non-centrosymmetric space group $\left(P 2_{1}\right)$, where most of the molecule is related by inversion symmetry except for the chiral carbon. This further contributes to the disorder and could not be improved to resolve the A- and B-alerts in the checkCIF for 3 . These facts should be taken into consideration when examining the bond metrics for complexes $\mathbf{1}$ and $\mathbf{3}$.

CCDC 2016367, 2016368, 2016369, and 2032420 contain the supplementary crystallographic data for this paper. These data are provided free of charge by The Cambridge Crystallographic Data Centre. 


\section{Crystallographic tables:}

Table S1. List of crystallographic parameters for compounds 1, 2, 3, and 19.

Compound

Empirical formula

Formula weight

Temperature/K

Crystal system

Space group

$\mathrm{a} / \AA$

$\mathrm{b} / \AA$

$\mathrm{c} / \AA$

$\alpha{ }^{\circ}$

$\beta /{ }^{\circ}$

$\gamma /{ }^{\circ}$

Volume $/ \AA^{3}$

$\mathrm{Z}$

$\rho_{\text {calc }} \mathrm{g} / \mathrm{cm}^{3}$

$\mu / \mathrm{mm}^{-1}$

$\mathrm{F}(000)$

Crystal size $/ \mathrm{mm}^{3}$

Radiation

$2 \Theta$ range for data collection/ ${ }^{\circ}$

Index ranges

Reflections collected

Independent reflections

Data/restraints/parameters

Goodness-of-fit on $\mathrm{F}^{2}$

Final R indexes $[\mathrm{I}>=2 \sigma(\mathrm{I})]$

Final $\mathrm{R}$ indexes [all data]

Largest diff. peak/hole / e $\AA^{-3}$

Flack parameter
1

$\mathrm{C}_{36} \mathrm{H}_{61} \mathrm{~N}_{5} \mathrm{O}_{2} \mathrm{SiZr}$

715.20

100

orthorhombic

Pbca

13.6685(5)

$16.3427(7)$

$35.6325(14)$

90

90

90

7959.6(5)

8

1.194

0.341

3056.0

$$
0.36 \times 0.22 \times 0.07
$$

$\operatorname{MoK} \alpha(\lambda=0.71073)$

3.756 to 48.312

$-15 \leq \mathrm{h} \leq 11,-18 \leq \mathrm{k} \leq 18,-40 \leq$

$$
\leq 40
$$

39455

$6362\left[\mathrm{R}_{\text {int }}=0.0766, \mathrm{R}_{\text {sigma }}=\right.$ $0.0574]$

$6362 / 0 / 423$

1.275

$\mathrm{R}_{1}=0.0703, \mathrm{wR}_{2}=0.1211$

$\mathrm{R}_{1}=0.0953, \mathrm{wR}_{2}=0.1275$

$0.82 /-1.35$
2

$\mathrm{C}_{39} \mathrm{H}_{63} \mathrm{~N}_{7} \mathrm{O}_{2} \mathrm{SiZr}$

781.27

100

monoclinic

$\mathrm{P} 2{ }_{1} / \mathrm{n}$

$10.8676(3)$

$20.4389(6)$

$18.9581(6)$

90

$90.480(2)$

90

4210.9(2)

4

1.232

0.330

1664.0

$0.34 \times 0.29 \times 0.14$

$\operatorname{MoK} \alpha(\lambda=0.71073)$

2.93 to 61.196

$-15 \leq \mathrm{h} \leq 14,-28 \leq \mathrm{k} \leq 29,-26 \leq$

$1 \leq 27$

50758

$12929\left[\mathrm{R}_{\text {int }}=0.0419, \mathrm{R}_{\text {sigma }}=\right.$ 0.0399]

$12929 / 0 / 464$

1.022

$\mathrm{R}_{1}=0.0487, \mathrm{wR}_{2}=0.1136$

$\mathrm{R}_{1}=0.0631, \mathrm{wR}_{2}=0.1224$

$1.90 /-1.81$ 
Compound

Empirical formula

Formula weight

Temperature/K

Crystal system

Space group

$\mathrm{a} / \AA$

$\mathrm{b} / \AA$

$\mathrm{c} / \AA$

$\alpha{ }^{\circ}$

$\beta /{ }^{\circ}$

$\gamma /{ }^{\circ}$

Volume $/ \AA^{3}$

Z

$\rho_{\text {calc }} \mathrm{g} / \mathrm{cm}^{3}$

$\mu / \mathrm{mm}^{-1}$

$\mathrm{F}(000)$

Crystal size $/ \mathrm{mm}^{3}$

Radiation

$2 \Theta$ range for data collection/ ${ }^{\circ}$

Index ranges

Reflections collected

Independent reflections

Data/restraints/parameters

Goodness-of-fit on $\mathrm{F}^{2}$

Final $\mathrm{R}$ indexes $[\mathrm{I}>=2 \sigma(\mathrm{I})]$

Final $\mathrm{R}$ indexes [all data]

Largest diff. peak/hole / e $\AA^{-3}$

Flack parameter
3

$$
\begin{gathered}
\mathrm{C}_{48} \mathrm{H}_{68} \mathrm{~N}_{6} \mathrm{O}_{2} \mathrm{SiZr} \\
880.39
\end{gathered}
$$

100

monoclinic

$\mathrm{P} 2{ }_{1}$

11.8111(7)

$39.5535(19)$

$12.1276(7)$

90

93.288(3)

90

$5656.3(5)$

4

1.034

0.252

1872.0

$0.21 \times 0.17 \times 0.11$

$\operatorname{MoK} \alpha(\lambda=0.71073)$

3.364 to 47.158

$$
-13 \leq \mathrm{h} \leq 11,-44 \leq \mathrm{k} \leq 44,-13
$$$$
\leq 1 \leq 12
$$

54817

$16833\left[\mathrm{R}_{\text {int }}=0.0494, \mathrm{R}_{\text {sigma }}=\right.$ $0.0604]$

$16833 / 1759 / 1059$

1.032

$\mathrm{R}_{1}=0.0603, \mathrm{wR}_{2}=0.1471$

$\mathrm{R}_{1}=0.0683, \mathrm{wR}_{2}=0.1514$

2.22/-1.93

0.5
19

$\mathrm{C}_{27} \mathrm{H}_{22} \mathrm{ClN}$

395.90

90

monoclinic

$\mathrm{C} 2 / \mathrm{c}$

33.6107(19)

$7.1095(4)$

$17.5870(10)$

90

108.2629(17)

90

$3990.8(4)$

8

1.318

0.205

1664.0

$0.18 \times 0.15 \times 0.14$

$\operatorname{MoK} \alpha(\lambda=0.71073)$

4.744 to 61.144

$-48 \leq \mathrm{h} \leq 41,-10 \leq \mathrm{k} \leq 10,-$ $25 \leq 1 \leq 25$

27057

$6097\left[\mathrm{R}_{\text {int }}=0.0448, \mathrm{R}_{\text {sigma }}=\right.$ $0.0415]$

$6097 / 0 / 262$

1.033

$\mathrm{R}_{1}=0.0441, \mathrm{wR}_{2}=0.1131$

$\mathrm{R}_{1}=0.0554, \mathrm{wR}_{2}=0.1205$

$0.64 /-0.62$ 
Figure S45. ORTEP representation of complex 1 with ellipsoids shown at 50\% probability and hydrogen atoms omitted for clarity. Selected bond lengths $(\AA)$ and angles $\left({ }^{\circ}\right)$ : Zr1-N1, 2.034(4); Zr1-C1, 2.322(5); N1-C8, 1.476(6); Si1-N1, 1.731(4); N1-Zr1-C1, 116.41(17); Si1-N1-Zr1, 135.1(2); N1-Zr1-C8, 30.65(15).

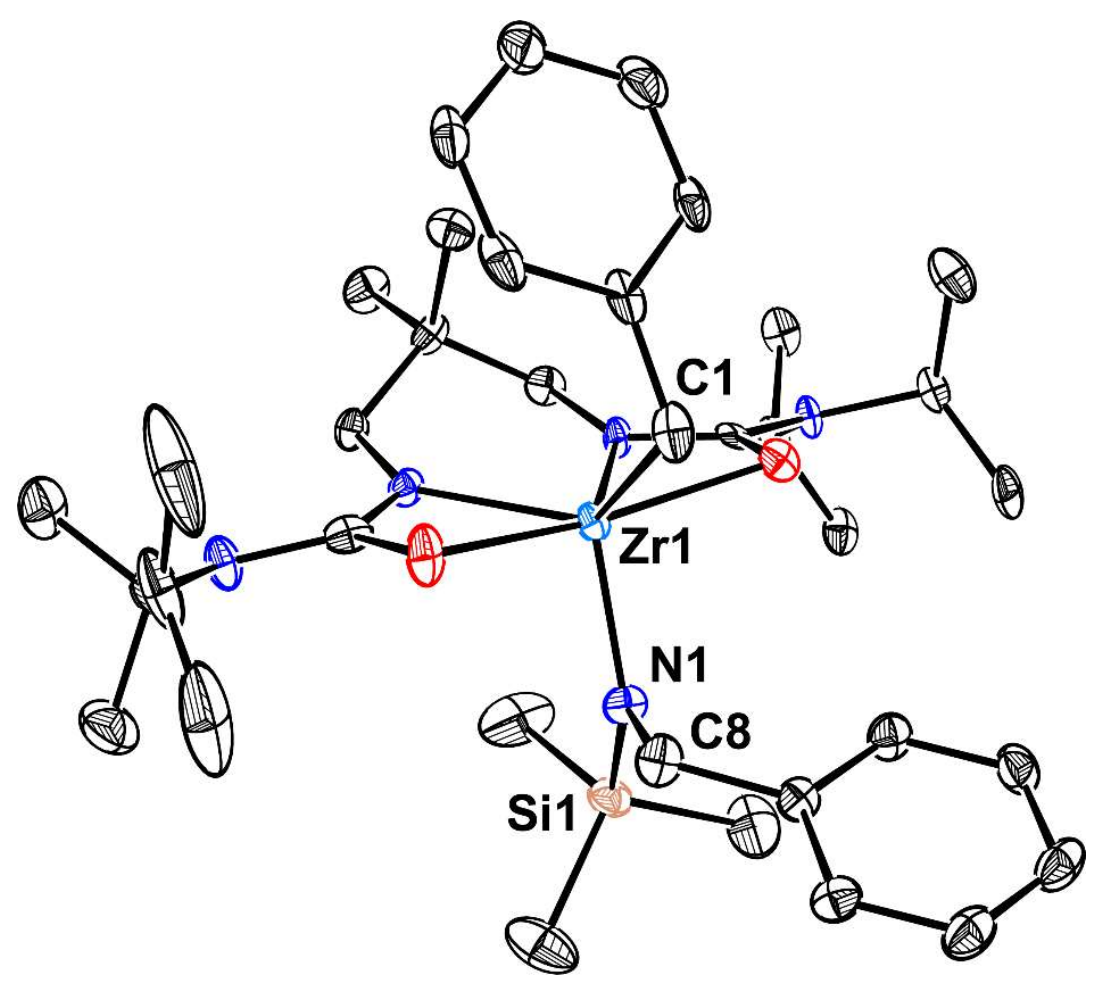


Figure S46. ORTEP representation of complex 2 with ellipsoids shown at 50\% probability and hydrogen atoms omitted for clarity. Selected bond lengths $(\AA)$ and angles $\left({ }^{\circ}\right)$ : Zr1-N1, 2.0600(17); $\mathrm{Zr1-C1}$, 2.322(2); N1-C1, 1.434(3); Zr1-N2, 2.4230(17); Zr1-N3, 2.5488(17); N1-Zr1-C1, 37.60(7); N1-Zr1-N2, 84.16(6); C1-Zr1-N2, 112.95(7); N2-Zr1-N3, 73.78(6), N1-Zr1-N3, 156.77(6); C1-Zr1-N3, 162.32(7).

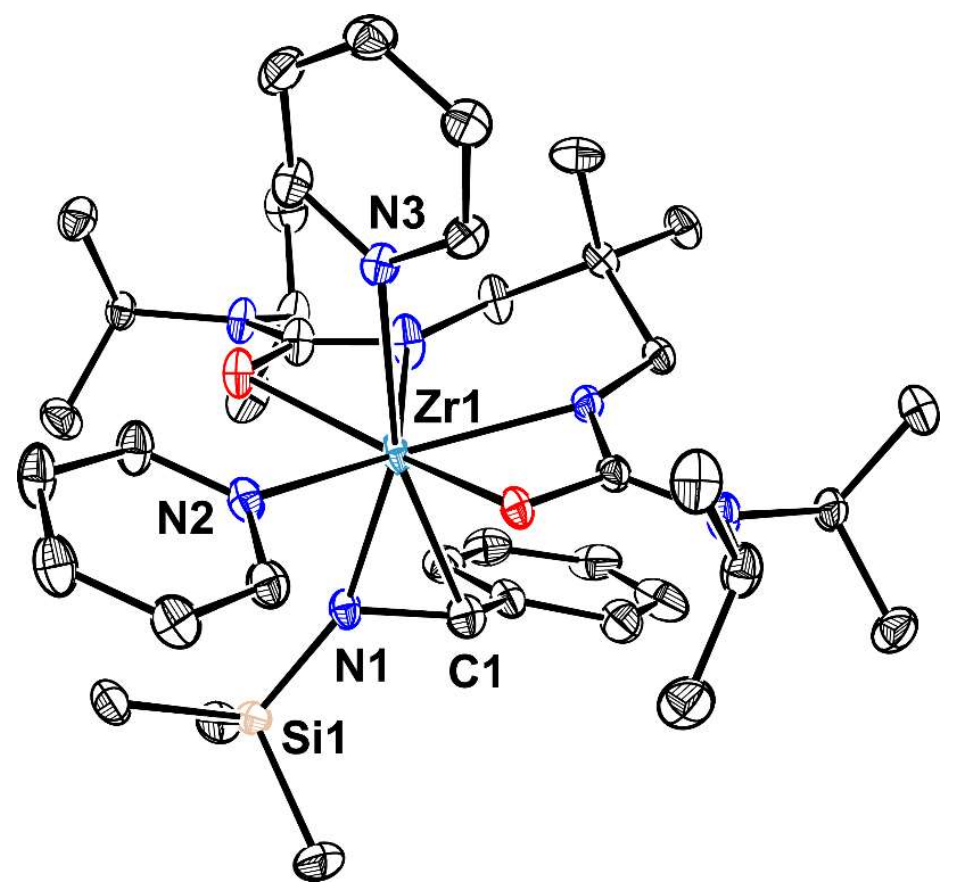


Figure S47. ORTEP representation of complex 3 with ellipsoids shown at 50\% probability and hydrogen atoms and $\mathrm{N}\left({ }^{i} \mathrm{Pr}\right)_{2}$ omitted for clarity. Selected bond lengths $(\AA)$ and angles $\left({ }^{\circ}\right)$ : Zr1-N1, 2.088(7); Zr1-C3, 2.314(9); Zr1-N2, 2.429(7); N1-C1, 1.49(1); C3-C2, 1.36(1); C1-C2, 1.53(1); $\mathrm{N} 1-\mathrm{Zr} 1-\mathrm{C} 3,76.9(3)$.

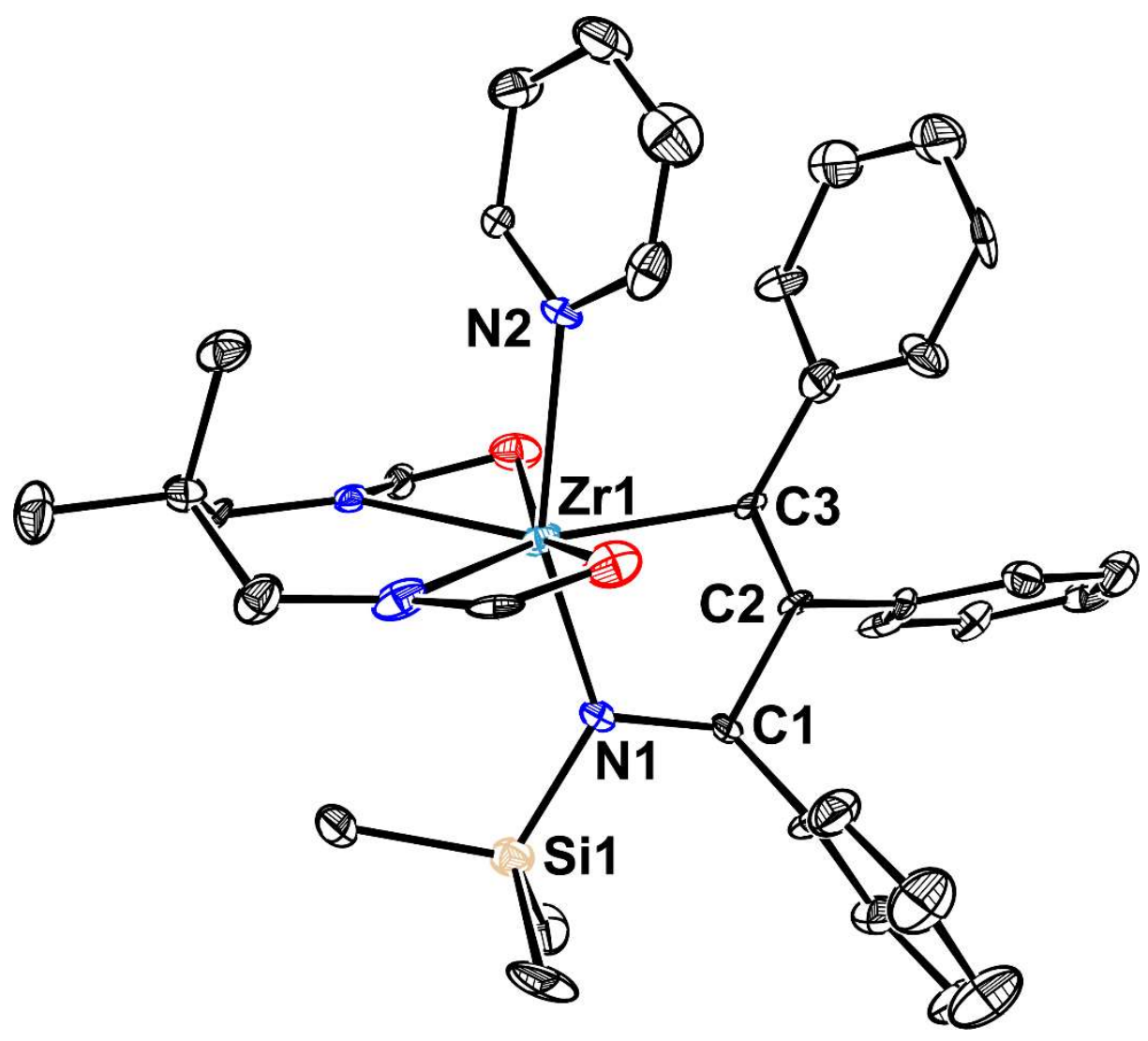


Figure S48. ORTEP representation of product 19 with ellipsoids shown at 50\% probability and aryl hydrogen atoms omitted for clarity. Selected bond lengths $(\AA)$ and angles $\left({ }^{\circ}\right)$ : N1-C7, 1.4635(16); C7-C14, 1.5316(18); C20-C14, 1.3450(18); C20-C14-C7, 120.70(11).

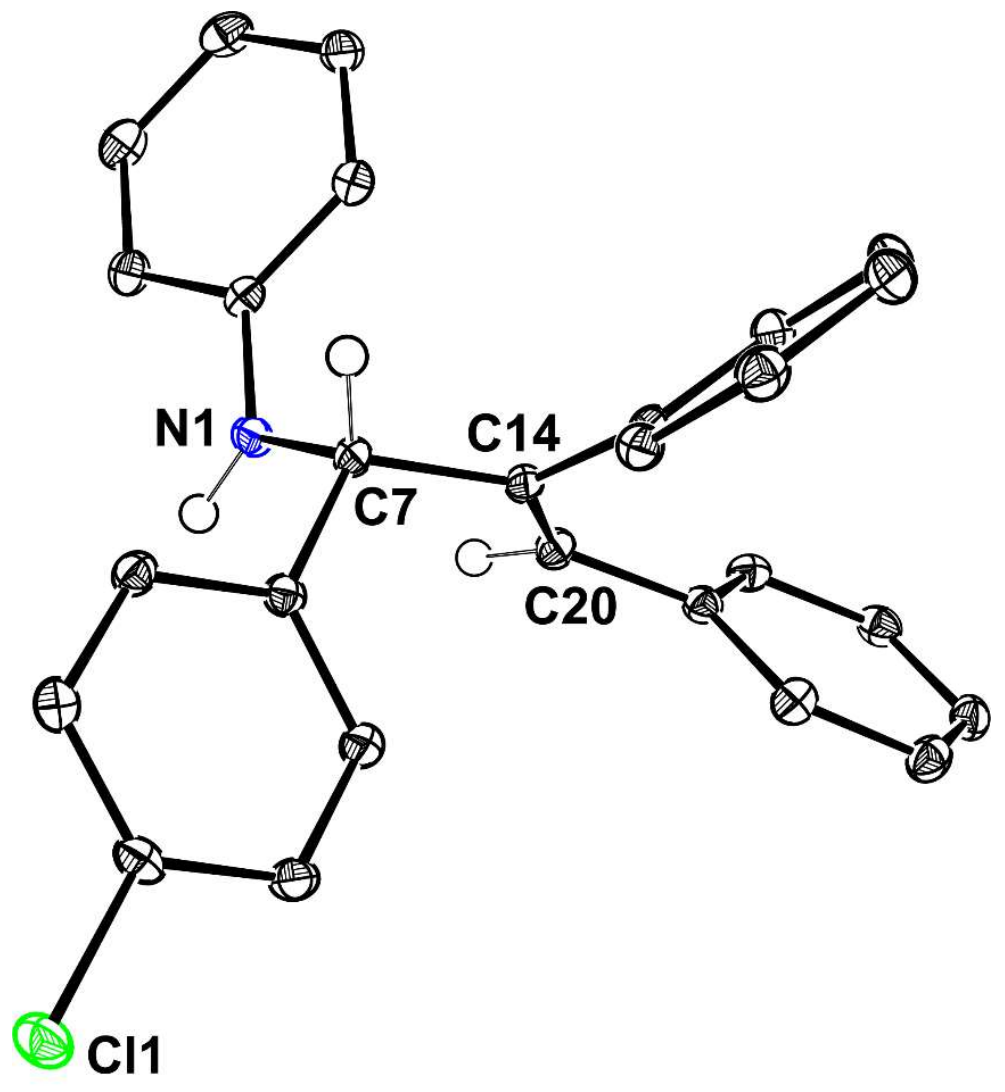




\section{References}

(1) Leitch, D. C.; Beard, J. D.; Thomson, R. K.; Wright, V. A.; Patrick, B. O.; Schafer, L. L. N,OChelates of Group 4 Metals: Contrasting the Use of Amidates and Ureates in the Synthesis of Metal Dichlorides. Eur. J. Inorg. Chem. 2009, 2691-2701.

(2) Leitch, D. C.; Schafer, L. L. Zirconium Alkyl Complexes Supported by Ureate Ligands: Synthesis, Characterization, and Precursors to Metal-Element Multiple Bonds. Organometallics 2010, 29, 5162-5172.

(3) DiPucchio, R. C.; Roşca, S.-C.; Schafer, L. L. Catalytic and Atom-Economic Csp3-Csp3 Bond Formation: Alkyl Tantalum Ureates for Hydroaminoalkylation. Angew. Chem., Int. Ed. 2018, 57, 3469-3472.

(4) Moorhouse, S.; Wilkinson, G. Bis[(Trimethylsilyl)Methyl]- and Bis(Neopentyl)-Zinc, and Tris[(Trimethylsilyl)Methyl]Aluminium-Diethyl Ether (1/1); Their Use as Alkylating Agents in Forming Niobium and Tantalum Alkyls. J. Chem. Soc., Dalton Trans. 1974, 2187-2190.

(5) Bielefeld, J.; Doye, S. Dimethylamine as a Substrate in Hydroaminoalkylation Reactions. Angew. Chem., Int. Ed. 2017, 56, 15155-15158.

(6) Fu, S.; Chen, N. Y.; Liu, X.; Shao, Z.; Luo, S. P.; Liu, Q. Ligand-Controlled Cobalt-Catalyzed Transfer Hydrogenation of Alkynes: Stereodivergent Synthesis of Z- and E-Alkenes. J. Am. Chem. Soc. 2016, 138, 8588-8594.

(7) Gregori, B. J.; Schwarzhuber, F.; Pöllath, S.; Zweck, J.; Fritsch, L.; Schoch, R.; Bauer, M.; Jacobi von Wangelin, A. Stereoselective Alkyne Hydrogenation by Using a Simple Iron Catalyst. ChemSusChem 2019, 12, 3864-3870.

(8) William, R.; Wang, S.; Ding, F.; Arviana, E. N.; Liu, X. W. Interrupted Imino-Nazarov Cyclization of 1-Aminopentadienyl Cation and Related Cascade Process. Angew. Chem., Int. Ed. 2014, 53, 10742-10746.

(9) Liu, Q.; Yu, S.; Hu, L.; Hussain, M. I.; Zhang, X.; Xiong, Y. Cross-Dehydrogenative Coupling Strategy for Phosphonation and Cyanation of Secondary N-Alkyl Anilines by Employing 2,3Dichloro-5,6-Dicyanobenzoquinone. Tetrahedron 2018, 74, 7209-7217.

(10) Griffin, S. E.; Pacheco, J.; Schafer, L. L. Reversible C-N Bond Formation in the ZirconiumCatalyzed Intermolecular Hydroamination of 2-Vinylpyridine. Organometallics 2019, 38, 10111016.

(11) Sakuramoto, T.; Donaka, Y.; Tobisu, M.; Moriuchi, T. Oxovanadium(v)-Catalyzed Deoxygenative Homocoupling Reaction of Alcohols. New J. Chem. 2019, 43, 17571-17576.

(12) Dolomanov, O. V.; Bourhis, L. J.; Gildea, R. J.; Howard, J. A. K.; Puschmann, H. OLEX2: A Complete Structure Solution, Refinement and Analysis Program. J. Appl. Cryst. 2009, 42, 339.

(13) Sheldrick, G. M. SHELXT - Integrated Space-Group and Crystal-Structure Determination. Acta Cryst. Sect. A 2015, 71, 3.

(14) Sheldrick, G. M. Crystal Structure Refinement with SHELXL. Acta Cryst. Sect. C 2015, 71, 3.

(15) Spek, A. L. PLATON SQUEEZE: A Tool for the Calculation of the Disordered Solvent Contribution to the Calculated Structure Factors. Acta Cryst. Sect. C 2015, 71, 9-18. 